LAWRENCE LIVERMORE NATIONAL LABORATORY

\title{
ENDF/B-VII.0 Data Testing Using 1,172 Critical Assemblies
}

\author{
by \\ Ernest F. Plechaty, LLNL \\ and \\ Dermott E. Cullen, LLNL \\ Contact \\ Dermott E. Cullen \\ University of California \\ Lawrence Livermore National Laboratory \\ P.O.Box 808/L-159 \\ Livermore, CA 94550 \\ Tele: 925-423-7359 \\ E.Mail: cullen1@llnl.gov \\ Website: $\underline{\text { http://home.comcast.net/ redcullen1 }}$
}

October 1, 2007

Approved for public release; further dissemination unlimited. 


\section{DISCLAIMER}

This document was prepared as an account of work sponsored by an agency of the United States government. Neither the United States government nor Lawrence Livermore National Security, LLC, nor any of their employees makes any warranty, expressed or implied, or assumes any legal liability or responsibility for the accuracy, completeness, or usefulness of any information, apparatus, product, or process disclosed, or represents that its use would not infringe privately owned rights. Reference herein to any specific commercial product, process, or service by trade name, trademark, manufacturer, or otherwise does not necessarily constitute or imply its endorsement, recommendation, or favoring by the United States government or Lawrence Livermore National Security, LLC. The views and opinions of authors expressed herein do not necessarily state or reflect those of the United States government or Lawrence Livermore National Security, LLC, and shall not be used for advertising or product endorsement purposes.

This work performed under the auspices of the U.S. Department of Energy by Lawrence Livermore National Laboratory under Contract DE-AC52-07NA27344. 
UCRL-TR-235178

\title{
ENDF/B-VII.0 Data Testing Using 1,172 Critical Assemblies
}

\author{
by \\ Ernest F. Plechaty, LLNL \\ and \\ Dermott E. Cullen, LLNL \\ Dermott E. Cullen \\ University of California \\ Lawrence Livermore National Laboratory \\ P.O.Box 808/L-159 \\ Livermore, CA 94550
}

Tele: 925-423-7359

E.Mail: cullen1@llnl.gov

Website: http://home.comcast.net/ redcullen1

October 1, 2007

\section{Introduction}

In order to test the ENDF/B-VII.0 neutron data library [1], 1,172 critical assemblies from [2] have been calculated using the Monte Carlo transport code TART [3]. TART's "best" physics was used for all of these calculations; this included continuous energy cross sections, delayed neutrons in their spectrum that is slower than prompt neutrons, unresolved resonance region self-shielding, the thermal scattering (free atom for all materials plus thermal scattering law data $\mathrm{S}(\alpha, \beta)$ when available).

In this first pass through the assemblies the objective was to "quickly" test the validity of the ENDF/B-VII.0 data [1], the assembly models as defined in [2] and coded for use with TART, and TART's physics treatment [3] of these assemblies. With TART we have the option of running criticality problems until K-eff has been calculated to an acceptable input accuracy. In order to "quickly" calculate all of these assemblies K-eff was calculated in each case to $+/-0.002$.

For these calculations the assemblies were divided into ten types based on fuel (mixed, Pu239, U233, U235) and median fission energy (Fast, Midi, Slow). The below table is a summary of these results. This is followed be details for every assembly, and statistical information about the distribution of K-eff for each type of assembly. 
After a review of these results to eliminate any obvious errors in ENDF/B data, assembly models, or TART physics, all assemblies will be run again to a higher precision. Only after this second run is finished will we have highly precise results. Until then the results presently here should only be interpreted as approximate values of K-eff with a standard deviation of +/- 0.002; for such a large number of assemblies we expected the results to be approximately normal, with a spread out to several times the standard deviation; see the below calculated statistical distributions and their comparisons to a normal distribution.

Based strictly on a "quick" look at the below summary my initial impression is that on average,

1) MIXED results look o.k.

2) PU239 fast results are very good., midi are too high, and slow is slightly too high.

3) U233 fast results are o.k., midi are poor, slow are o.k.

1) U235 fast results are good, midi too high, and slow very good.

\begin{tabular}{|l|l|l|l|l|}
\hline Type & Assemblies & Average & Minimum & Maximum \\
\hline Mixed & 57 & $1.00177+/-0.00459$ & 0.98546 & 1.01039 \\
Pu Fast & 48 & $1.00016+/-0.00462$ & 0.98297 & 1.01099 \\
Pu Midi & 36 & $1.01896+/-0.00769$ & 0.99689 & 1.03538 \\
Pu Slow & 247 & $1.00489+/-0.00627$ & 0.98271 & 1.04091 \\
U233 Fast & 12 & $1.00226+/-0.00485$ & 0.99401 & 1.01066 \\
U233 Midi & 33 & $0.98193+/-0.00589$ & 0.96787 & 0.99999 \\
U233 Slow & 155 & $0.99798+/-0.00808$ & 0.96628 & 1.02051 \\
U235 Fast & 199 & $1.00101+/-0.00523$ & 0.97584 & 1.02526 \\
U235 Midi & 13 & $1.00429+/-0.00613$ & 0.99200 & 1.01276 \\
U235 Slow & 372 & $0.99999+/-0.00606$ & 0.97809 & 1.03050 \\
Total & 1172 & & & \\
\hline
\end{tabular}

\section{References}

[1] "ENDF/B-VII.0: Next Generation Evaluated Nuclear Data Library for Nuclear Science and Technology”, Nuclear Data Sheets 107 (2006) pp. 2931-3060, Editors: P.Oblozinsky and M.Herman.

[2] "International Handbook of Evaluated Criticality Safety Benchmark Experiments", NEA/NSC/DOC(95)03, September 2006 edition, Editor Blair Briggs

[3] .TART 2005: A Coupled Neutron-Photon 3-D, Time Dependent, Combinatorial Geometry Monte Carlo Transport Code," Lawrence Livermore National Laboratory, UCRLSM-218009, November 22, 2005, by D.E.Cullen. 


\section{Mixed (57 Assemblies)}

Criticality Calculation Editor (CRITEDIT 2002-1)

\begin{tabular}{|c|c|c|c|c|c|c|c|}
\hline $\begin{array}{l}\text { Crit. } \\
\text { ID. }\end{array}$ & Fuel & $\begin{array}{l}\text { Reflector } \\
\text { or Name }\end{array}$ & Expected $\mathrm{K}$ & $\begin{array}{l}\text { Removal } \\
\text { Lifetime } \\
\text { (Microsec.) }\end{array}$ & $\begin{array}{l}\text { Median } \\
\text { Energy } \\
(\text { MeV })\end{array}$ & $\begin{array}{l}\text { Average } \\
\text { Energy } \\
(\mathrm{MeV})\end{array}$ & Seconds \\
\hline
\end{tabular}

\begin{tabular}{|c|c|c|c|c|c|c|c|}
\hline MMF००1-1 & Pu239 & U235 & ๑. 9971610 & $4.38003 \mathrm{D}-\odot 3$ & $1.32817 \mathrm{D}+\Theta \odot$ & $1.81656 \mathrm{D}+00$ & 1.280 \\
\hline MMF○०2 - 1 & Pu239 & Flattop & 1.0036100 & $7.08866 \mathrm{D}-\odot 2$ & $1.14973 D+\odot \odot$ & $1.74642 \mathrm{D}+\odot \odot$ & 8.200 \\
\hline MMF๑०2 - 2 & Pu239 & Flattop & 1.0080500 & $7.02324 \mathrm{D}-\odot 2$ & $1.11442 \mathrm{D}+\odot \odot$ & $1.72515 \mathrm{D}+\odot \odot$ & 9.920 \\
\hline MMF००2 - 3 & Pu239 & Flattop & 1.0047800 & 7. $03334 \mathrm{D}-02$ & 1. $\odot 9901 \mathrm{D}+\odot \odot$ & $1.71424 \mathrm{D}+\odot \odot$ & 8.660 \\
\hline MMF○०3-1 & Pu239 & U235 & 1.0052100 & $9.57130 \mathrm{D}-\odot 3$ & $1.30107 \mathrm{D}+00$ & $1.80289 \mathrm{D}+0 \odot$ & 5.980 \\
\hline MMF○๑4 - 1 & Pu239 & U235-Be & $\odot .9918450$ & 1. 11663D- 02 & $9.80256 \mathrm{D}-01$ & $1.54773 \mathrm{D}+\odot \odot$ & 8.610 \\
\hline MMF๑๑4- 2 & Pu239 & U235-BeO & 1.0026300 & $1.13768 \mathrm{D}-02$ & $9.45068 \mathrm{D}-01$ & $1.52077 \mathrm{D}+00$ & 10.480 \\
\hline MMF๑๑5 - 1 & Pu239 & U235-Al & 1.0048500 & 1. 16771D- - 2 & $1.25412 \mathrm{D}+\odot \odot$ & $1.75748 \mathrm{D}+\odot \odot$ & 7.810 \\
\hline MMF○๑7-1 & Pu239 & U235-Be & 1.0073600 & $1.29764 \mathrm{D}+02$ & $1.16054 \mathrm{D}+\odot \odot$ & $1.88986 \mathrm{D}+0 \odot$ & 14.830 \\
\hline MMF○९7 - 2 & Pu239 & U235-Be & 1.0060500 & $5.80758 D+01$ & $1.10513 \mathrm{D}+00$ & $1.84419 D+0 \odot$ & 9.220 \\
\hline MMF○๑7 - 3 & Pu239 & U235-Be & $\odot .9987300$ & $2 . \odot 4635 D+0 \odot$ & $1.12771 \mathrm{D}+\odot \odot$ & $1.80336 \mathrm{D}+\odot \odot$ & 3.250 \\
\hline MMF๑๑7 - 4 & Pu239 & U235-Be & 1.0061900 & $1.18387 D-01$ & $1.11476 \mathrm{D}+\odot \odot$ & $1.76573 \mathrm{D}+\odot \odot$ & 1.480 \\
\hline MMF๑๑7 - 5 & Pu239 & $\mathrm{U} 235-\mathrm{Be}$ & 1.0003900 & $1.12577 D-\odot 2$ & $1.10687 \mathrm{D}+\odot \odot$ & $1.70115 \mathrm{D}+00$ & 1.170 \\
\hline MMF○०7- 6 & Pu239 & U235-Be & 1.0013000 & $7.06356 \mathrm{D}-03$ & 1. $\odot 8159 D+\odot \odot$ & $1.65300 \mathrm{D}+0 \odot$ & 1.420 \\
\hline MMF○९7 - 7 & Pu239 & $\mathrm{U} 235-\mathrm{Be}$ & 1.0034000 & $7.58701 \mathrm{D}+01$ & $1.33050 \mathrm{D}+\odot \odot$ & $1.98912 \mathrm{D}+\odot \odot$ & 11.450 \\
\hline MMF๑๑7 - 8 & Pu239 & $\mathrm{U} 235-\mathrm{Be}$ & 1.0008200 & $2.21177 D+\odot 1$ & $1.3086 \odot \mathrm{D}+\odot \odot$ & $1.96387 \mathrm{D}+00$ & 5.730 \\
\hline MMF००7 - 9 & Pu239 & U235-Be & 1.0103900 & $7.38217 \mathrm{D}+\odot \odot$ & $1.29160 \mathrm{D}+0 \odot$ & $1.92048 \mathrm{D}+00$ & 3.200 \\
\hline MMF $\odot \odot 7-10$ & Pu239 & $\mathrm{U} 235-\mathrm{Be}$ & 1.0032600 & $1.64255 \mathrm{D}-01$ & $1.21980 \mathrm{D}+\odot \odot$ & $1.83900 \mathrm{D}+\odot \odot$ & 1.890 \\
\hline MMF $007-11$ & Pu239 & $\mathrm{U} 235-\mathrm{Be}$ & 1.0077500 & 1. 98269D-๑2 & $1.17812 \mathrm{D}+\odot \odot$ & $1.78188 \mathrm{D}+\odot \odot$ & 1.520 \\
\hline MMF○๑7-12 & Pu239 & U235-Be & ๑. 9976510 & 7. 4०829D-๑3 & $1.14734 \mathrm{D}+\odot \odot$ & $1.71217 \mathrm{D}+\odot \odot$ & 1.220 \\
\hline MMF๑๑7-13 & Pu239 & $\mathrm{U} 235-\mathrm{Be}$ & $\odot .9998860$ & $5.66745 \mathrm{D}-\odot 3$ & $1.17061 \mathrm{D}+\odot \odot$ & $1.70934 \mathrm{D}+00$ & 1.000 \\
\hline MMF○०7-14 & Pu239 & U235-Be & 1.0057000 & $5.10753 \mathrm{D}+\odot \odot$ & $1.42589 \mathrm{D}+00$ & $2.00917 \mathrm{D}+00$ & 2.580 \\
\hline MMF๑๑7 - 15 & Pu239 & $\mathrm{U} 235-\mathrm{Be}$ & 1.0043500 & $1.59091 \mathrm{D}+\odot \odot$ & $1.3546 \odot \mathrm{D}+\odot \odot$ & $1.97690 \mathrm{D}+\odot \odot$ & 2.390 \\
\hline MMF○๑7-16 & Pu239 & $\mathrm{U} 235-\mathrm{Be}$ & 1.0084100 & $3.29638 D-02$ & $1.29793 \mathrm{D}+\odot \odot$ & $1.88181 \mathrm{D}+\odot \odot$ & 1.700 \\
\hline MMF○๑7-17 & Pu239 & $\mathrm{U} 235-\mathrm{Be}$ & 1.0021800 & 1. $05610 \mathrm{D}-02$ & $1.24821 \mathrm{D}+\odot \odot$ & $1.82054 \mathrm{D}+0 \odot$ & $\odot .910$ \\
\hline MMF๑๑7 - 18 & Pu239 & U235-Be & $1.009240 \odot$ & $6.14974 \mathrm{D}-03$ & $1.18857 \mathrm{D}+\odot \odot$ & $1.72665 \mathrm{D}+\odot \odot$ & $\odot .880$ \\
\hline MMF007-19 & Pu239 & $\mathrm{U} 235-\mathrm{Be}$ & 1.0000500 & $1.56927 D-02$ & $1.32964 \mathrm{D}+00$ & $1.88239 \mathrm{D}+0 \odot$ & 1.550 \\
\hline MMF○०7-20 & Pu239 & $\mathrm{U} 235-\mathrm{Be}$ & 1.0042300 & $7.43490 \mathrm{D}-03$ & $1.27665 \mathrm{D}+0 \odot$ & $1.81612 \mathrm{D}+\odot \odot$ & 1.120 \\
\hline MMF○๑7- 21 & Pu239 & $\mathrm{U} 235-\mathrm{Be}$ & 1.0054200 & $5.0 \odot 833 D-\odot 3$ & $1.25675 \mathrm{D}+\odot \odot$ & $1.77863 \mathrm{D}+\odot \odot$ & 1.060 \\
\hline MMF○๑7- 22 & Pu239 & $\mathrm{U} 235-\mathrm{Be}$ & 1.0022000 & 8. 84976D-๑3 & $1.37653 \mathrm{D}+\odot \odot$ & $1.9 \odot 873 \mathrm{D}+\odot \odot$ & 1.380 \\
\hline MMF๑๑7- 23 & Pu239 & U235-Be & 1.0039300 & $5.782 \odot 7 \mathrm{D}-\odot 3$ & $1.31546 \mathrm{D}+\odot \odot$ & $1.85108 \mathrm{D}+\odot \odot$ & 1.080 \\
\hline MMFO०9-1 & Pu239 & U235 & 1.0035100 & $4.22796 \mathrm{D}-03$ & 1. $39024 D+\odot \odot$ & $1.86866 \mathrm{D}+\odot \odot$ & 3.200 \\
\hline MMF010-1 & Pu239 & U235 & $\odot .9988840$ & $5.32506 \mathrm{D}-\odot 3$ & $1.19806 \mathrm{D}+00$ & $1.70025 \mathrm{D}+\odot \odot$ & 4.730 \\
\hline MMF011-1 & Pu239 & U235-Graphite & $\odot .9959260$ & $3.96329 \mathrm{D}+\odot \odot$ & $5.38270 \mathrm{D}-01$ & $1 . \odot 7525 \mathrm{D}+\odot \odot$ & 144.580 \\
\hline MMF011-2 & U235 & Pu-Graphite & 1.0001400 & $3.14363 \mathrm{D}+\odot \odot$ & $5.14783 \mathrm{D}-01$ & $1 . \odot 4911 \mathrm{D}+\odot \odot$ & 106.530 \\
\hline MMF011-3 & U235 & Pu-Graphite & 1.0066500 & $4.84409 \mathrm{D}+0 \odot$ & $4.14988 D-01$ & OD- 01 & 140.300 \\
\hline MMF011- 4 & U235 & Pu-Graphite & 1.0078000 & $3.42279 D+\odot \odot$ & $4.15068 D-01$ & 9.438 & 139.530 \\
\hline MCF००1 & U235 & ZPR6/7 & $\odot .9875050$ & $6.80559 D-01$ & $1.64005 \mathrm{D}-01$ & $8.52485 D-01$ & 157.840 \\
\hline Msto०6-1s & Pu239u235 & $\mathrm{H} 2 \mathrm{O}$ & $\odot .9854610$ & $3.906 \odot 4 \mathrm{D}+\odot 1$ & $8.63649 D-\odot 8$ & $3.66694 \mathrm{D}-02$ & 207.310 \\
\hline Msto06-2s & Pu239U235 & $\mathrm{H} 2 \mathrm{O}$ & $\odot .9905270$ & $3.41540 \mathrm{D}+01$ & 1. $03639 D-\odot 7$ & $3.87693 \mathrm{D}-\odot 2$ & 215.420 \\
\hline Mst006-3s & Pu239u235 & $\mathrm{H} 2 \mathrm{O}$ & $\odot .9955720$ & $3.19877 D+01$ & $1.09685 \mathrm{D}-07$ & $4.71316 \mathrm{D}-\odot 2$ & 123.890 \\
\hline Mst006-4s & Pu239U235 & $\mathrm{H} 2 \mathrm{O}$ & 1. 0000100 & $2.84056 \mathrm{D}+01$ & $1.23660 \mathrm{D}-07$ & 405D- - 2 & 173.060 \\
\hline Mst006-5s & Pu239U235 & $\mathrm{H} 2 \mathrm{O}$ & 1.0058600 & $2.65535 \mathrm{D}+01$ & $1.26456 \mathrm{D}-07$ & 4.18924D- 02 & 114.250 \\
\hline Mste०6-6s & Pu239u235 & $\mathrm{H} 2 \mathrm{O}$ & 1.0024100 & $2.55762 \mathrm{D}+01$ & 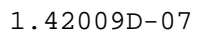 & $4.97231 D-\odot 2$ & 165.810 \\
\hline Mst006-1 & Pu239u235 & $\mathrm{H} 2 \mathrm{O}$ & $\odot .9855750$ & $3.88704 \mathrm{D}+01$ & $9.22318 D-\odot 8$ & $3.97859 D-\odot 2$ & 241.670 \\
\hline Mst@०6-2 & Pu239u235 & $\mathrm{H} 2 \mathrm{O}$ & $\odot .9980710$ & $3.36931 D+01$ & $1.07017 \mathrm{D}-07$ & $4.33410 \mathrm{D}-02$ & 208.220 \\
\hline Mst@०6-3 & Pu239u235 & $\mathrm{H} 2 \mathrm{O}$ & $\odot .9949630$ & $3.23524 \mathrm{D}+01$ & $1.15568 \mathrm{D}-07$ & $4.29141 D-\odot 2$ & 161.500 \\
\hline Mst@๑6-4 & Pu239U235 & $\mathrm{H} 2 \mathrm{O}$ & 1.0006800 & $2.85041 \mathrm{D}+01$ & $1.29496 \mathrm{D}-07$ & 4.29879D-02 & 147.720 \\
\hline Mst@๑6-5 & Pu239u235 & $\mathrm{H} 2 \mathrm{O}$ & 1.0060500 & $2.68264 \mathrm{D}+01$ & $1.36861 \mathrm{D}-07$ & $4.20212 \mathrm{D}-\odot 2$ & 169.380 \\
\hline Mst@०6-6 & Pu239U235 & $\mathrm{H} 2 \mathrm{O}$ & 1.0063700 & $2.60554 \mathrm{D}+\odot 1$ & $1.41629 \mathrm{D}-07$ & $4.65599 D-02$ & 133.310 \\
\hline MSTO०7 - 1 & Pu239u235 & $\mathrm{H} 2 \mathrm{O}$ & $\odot .9895950$ & $8.58150 D+01$ & $8.72348 D-08$ & $2.96214 \mathrm{D}-\odot 2$ & 96.120 \\
\hline MSTO०7 - 2 & Pu239U235 & $\mathrm{H} 2 \mathrm{O}$ & 0.9991930 & $7.66135 D+01$ & $6.96435 \mathrm{D}-08$ & $3.11383 \mathrm{D}-02$ & 86.480 \\
\hline MSTO०7 - 3 & Pu239U235 & $\mathrm{H} 2 \mathrm{O}$ & 1.0033600 & $5.89231 D+01$ & 1. 10880D-०7 & $3.23339 \mathrm{D}-02$ & 54.110 \\
\hline 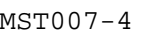 & Pu239u235 & $\mathrm{H} 2 \mathrm{O}$ & 1.0102400 & $5.34664 \mathrm{D}+01$ & $1.19467 \mathrm{D}-07$ & $3.19402 \mathrm{D}-\odot 2$ & 73.450 \\
\hline MST०९7 - 5 & Pu239U235 & $\mathrm{H} 2 \mathrm{O}$ & 1.0070700 & $4.84022 \mathrm{D}+01$ & $1.20563 \mathrm{D}-07$ & $3.00682 D-\odot 2$ & 48.940 \\
\hline 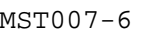 & Pu239u235 & $\mathrm{H} 2 \mathrm{O}$ & 1.0065400 & $3.82157 D+01$ & $1.43372 \mathrm{D}-07$ & $3.43447 \mathrm{D}-\odot 2$ & 45.700 \\
\hline MSTO०7 - 6 & Pu239U235 & $\mathrm{H} 2 \mathrm{O}$ & 1.0058700 & $3.51961 D+01$ & 1.51881D-07 & $3.88879 \mathrm{D}-02$ & 43.660 \\
\hline & & & & E=ニ=ニ= & E=====- & $===$ & $:==$ \\
\hline
\end{tabular}




$\begin{array}{lrr}\text { Lowest } & 0.9854610 & -0.0163084 \text { (from Average) } \\ \text { Highest } & 1.0103900 & 0.0086206 \text { (from Average) }\end{array}$

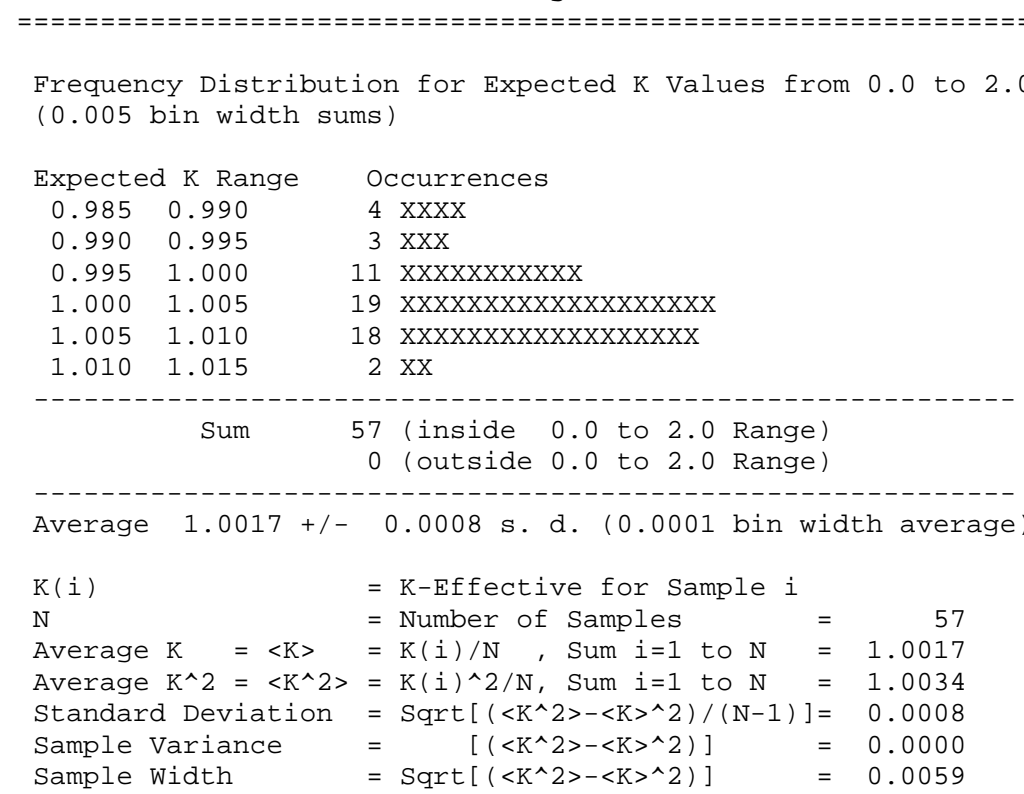

As N Approaches Infinity Standard Deviation Approaches Zero. As $\mathrm{N}$ Approaches Infinity Sample Width Approaches a Constant.

Confidence Limits

(Occurrences out to $+/-10$ times Sample Width)

\begin{tabular}{lrrrr}
$\begin{array}{l}\text { Sample Width } \\
\text { Range }\end{array}$ & 0ccurrences & $\begin{array}{r}\text { Per-Cent } \\
\text { Occurred }\end{array}$ & $\begin{array}{r}\text { Per-Cent } \\
\text { Normal }\end{array}$ \\
-3 & -2 & 4 & 7.018 & 2.140 \\
-2 & -1 & 4 & 7.018 & 13.591 \\
-1 & 0 & 15 & 26.316 & 34.134 \\
$\odot$ & 1 & 27 & 47.368 & 34.134 \\
1 & 2 & 7 & 12.281 & 13.591 \\
- & \multicolumn{2}{c}{ Sum } & 57 &
\end{tabular}




\section{Pu Fast (48 Assemblies)}

Criticality Calculation Editor (CRITEDIT 2002-1)

\begin{tabular}{|c|c|c|c|c|c|c|c|}
\hline $\begin{array}{l}\text { Crit. } \\
\text { ID. }\end{array}$ & Fuel & $\begin{array}{l}\text { Reflector } \\
\text { or Name }\end{array}$ & Expected $\mathrm{K}$ & $\begin{array}{l}\text { Removal } \\
\text { Lifetime } \\
\text { (Microsec.) }\end{array}$ & $\begin{array}{l}\text { Median } \\
\text { Energy } \\
(\mathrm{MeV})\end{array}$ & $\begin{array}{l}\text { Average } \\
\text { Energy } \\
(\mathrm{MeV})\end{array}$ & Seconds \\
\hline \multicolumn{8}{|c|}{ 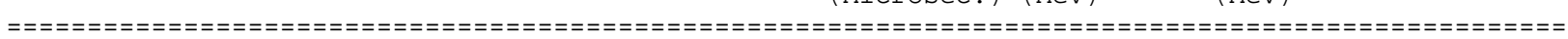 } \\
\hline PMF001-1 & Pu239 & Jezebel & 1.0059300 & $3.73490 \mathrm{D}-\odot 3$ & $1.45141 \mathrm{D}+00$ & $1.92138 \mathrm{D}+0 \odot$ & 0.770 \\
\hline PMF००2 - 1 & Pu239 & Jezebel & 1.0034400 & $4.03351 D-\odot 3$ & $1.46692 \mathrm{D}+\odot \odot$ & $1.9260 \odot \mathrm{D}+\odot \odot$ & 0.700 \\
\hline PMF००5-1 & Pu239 & W & 1.0109900 & 1. 4०337D-๑2 & $1.20515 \mathrm{D}+\odot \odot$ & $1.73145 \mathrm{D}+\odot \odot$ & 5.520 \\
\hline PMF००6 & Pu239 & Flattop & 0.9968470 & $7.74139 \mathrm{D}-02$ & $1.50454 \mathrm{D}+\odot \odot$ & $2.00353 \mathrm{D}+\odot \odot$ & 7.000 \\
\hline PMF๑๑8-1 & Pu239 & Thorium & $\odot .9974590$ & $7.48934 \mathrm{D}-02$ & 1. $35230 D+\odot \odot$ & $1.92913 \mathrm{D}+\odot \odot$ & 3.170 \\
\hline PMF००8-2 & Pu239 & Thorium & $\odot .9933360$ & $7.34728 D-02$ & $1.3397 \odot \mathrm{D}+\odot \odot$ & $1.91991 \mathrm{D}+0 \odot$ & 2.890 \\
\hline PMF००9-1 & Pu239 & Aluminum & 1.0091300 & 1. 42369D-๑2 & $1.32441 \mathrm{D}+\odot \odot$ & $1.80158 \mathrm{D}+0 \odot$ & 2.920 \\
\hline PMF010-1 & Pu239 & \multirow{2}{*}{$\begin{array}{l}\text { U-nat } \\
\text { water }\end{array}$} & $\odot .9979740$ & 1. $\odot 4398 D-\odot 2$ & $1.45691 \mathrm{D}+\odot \odot$ & $1.94415 \mathrm{D}+\odot \odot$ & 1.660 \\
\hline PMF011-1 & Pu239 & & 1.0037300 & $1.20424 \mathrm{D}+02$ & $1.04233 \mathrm{D}+00$ & $1.51977 \mathrm{D}+0 \odot$ & 38.080 \\
\hline PMF018-1 & Pu239 & & 0.9947810 & $1.64912 \mathrm{D}-02$ & 1. $44281 \mathrm{D}+00$ & $1.97997 \mathrm{D}+\odot \odot$ & 1.390 \\
\hline PMF019-1 & Pu239 & $\mathrm{Be}$ & 1.0014700 & $1.23468 \mathrm{D}-01$ & $1.43136 \mathrm{D}+\odot \odot$ & $1.99487 \mathrm{D}+\odot \odot$ & 13.140 \\
\hline PMF०20-1 & Pu239 & U238 & $\odot .9966390$ & $2.49421 \mathrm{D}-\odot 2$ & $1.47421 \mathrm{D}+\odot \odot$ & $1.95757 \mathrm{D}+\odot \odot$ & 7.050 \\
\hline PMF021-1 & Pu239 & \multirow{2}{*}{$\begin{array}{l}\mathrm{Be} \\
\mathrm{BeO}\end{array}$} & 0.9995220 & 8. 95677D-01 & $1.45295 \mathrm{D}+00$ & $2.02375 D+0 \odot$ & 8.330 \\
\hline PMF021-2 & Pu239 & & $\odot .9928220$ & 4. 93097D-01 & $1.37627 \mathrm{D}+0 \odot$ & $1.91321 \mathrm{D}+0 \odot$ & 9.120 \\
\hline PMF022-S & Pu239 & bare & $\odot .9995710$ & $3.92425 \mathrm{D}-03$ & $1.45215 \mathrm{D}+\odot \odot$ & $1.89743 \mathrm{D}+\odot \odot$ & 2.980 \\
\hline PMF022-D & Pu239 & bare & 1.0018000 & $3.95398 \mathrm{D}-\odot 3$ & $1.43880 \mathrm{D}+\odot \odot$ & $1.90333 \mathrm{D}+0 \odot$ & 4.980 \\
\hline PMFO23-S & PU239 & Graphite & 1.0015400 & $7.05910 \mathrm{D}-\odot 3$ & $1.36909 \mathrm{D}+\odot \odot$ & $1.82327 \mathrm{D}+\odot \odot$ & 3.670 \\
\hline PMF023 - D & PU239 & Graphite & 0.9969920 & $7.02470 \mathrm{D}-\odot 3$ & $1.33724 \mathrm{D}+00$ & $1.81243 \mathrm{D}+0 \odot$ & 3.230 \\
\hline PMF024-S & Pu239 & $\begin{array}{l}\text { poly } \\
\text { Poly }\end{array}$ & 1.0056600 & $1.50569 \mathrm{D}-01$ & $1.29723 D+\odot \odot$ & $1.75657 \mathrm{D}+\odot \odot$ & 3.700 \\
\hline PMF024 - D & Pu239 & $\begin{array}{l}\text { Poly } \\
\text { steel }\end{array}$ & 1.0008700 & 1. $6 \odot 445 \mathrm{D}-01$ & $1.29719 D+0 \odot$ & $1.75069 \mathrm{D}+0 \odot$ & 4.750 \\
\hline PMF०25-S & Pu239 & steel & $\odot .997524 \odot$ & $5.44868 \mathrm{D}-\odot 3$ & $1.38109 \mathrm{D}+\odot \odot$ & $1.83778 \mathrm{D}+\odot \odot$ & 5.940 \\
\hline PMFO25 - D & Pu239 & Steel & 1.0011900 & $5.47255 \mathrm{D}-03$ & $1.40997 D+0 \odot$ & $1.85495 \mathrm{D}+0 \odot$ & 4.120 \\
\hline PMF026-S & Pu239 & steel & 0.9945090 & $2.64149 \mathrm{D}-02$ & $1.28512 \mathrm{D}+00$ & $1.74639 \mathrm{D}+\odot \odot$ & 21.200 \\
\hline PMF०26 - D & Pu239 & Steel & 69320 & $2.71058 \mathrm{D}-02$ & $7307 D+\odot \odot$ & $1.75675 \mathrm{D}+\odot \odot$ & 15.620 \\
\hline PMF०27-S & Pu239 & Poly & 1.0036500 & $1.78305 \mathrm{D}+01$ & $9.99689 \mathrm{D}-01$ & $1.48397 \mathrm{D}+0 \odot$ & 12.050 \\
\hline PMF०27 - D & Pu239 & Steel & 1. $0 \odot 7170 \odot$ & $1.76829 D+01$ & 1. $\odot \odot 925 D+\odot \odot$ & $1.48375 \mathrm{D}+\odot \odot$ & 15.220 \\
\hline SFIRO०1 & Pu239 & Jezebel & 1.0034000 & $3.76897 \mathrm{D}-03$ & $1.46266 \mathrm{D}+\odot \odot$ & $1.92492 \mathrm{D}+\odot \odot$ & 0.780 \\
\hline SFIR๑०2 & Pu239 & Vera-11a & $\odot .9829740$ & $2.12080 \mathrm{D}-01$ & 7. 59629D-01 & $1.43472 \mathrm{D}+\odot \odot$ & 91.670 \\
\hline SFIR००8 & Pu239 & Thor & 0.9943780 & $7.58576 \mathrm{D}-02$ & $1.33997 \mathrm{D}+\odot \odot$ & $1.9 \odot 258 \mathrm{D}+\odot \odot$ & 3.390 \\
\hline SFIR००9 & Pu239 & Flattop & 1.0065300 & $7.67354 \mathrm{D}-02$ & $1.49419 \mathrm{D}+00$ & $1.99054 \mathrm{D}+00$ & 10.910 \\
\hline SFIR010 & Pu239 & Zebra-3 & $\odot .9914470$ & 1. $60798 \mathrm{D}-\odot 1$ & $5.66365 D-01$ & 1. $45133 \mathrm{D}+\odot \odot$ & 129.520 \\
\hline SFIR013 & Pu239 & Jezebel & ๑.9905260 & $3.98341 \mathrm{D}-\odot 3$ & $1.46433 \mathrm{D}+00$ & $1.93339 \mathrm{D}+0 \odot$ & 1.060 \\
\hline PMF028-S & Pu239 & Steel & $\odot .9956340$ & $7.75285 \mathrm{D}-\odot 2$ & $1.27744 \mathrm{D}+00$ & $1.74331 \mathrm{D}+0 \odot$ & 27.750 \\
\hline PMF028-D & Pu239 & Steel & 23800 & $7.42221 \mathrm{D}-02$ & $1.28094 \mathrm{D}+\odot \odot$ & $1.74561 \mathrm{D}+\odot \odot$ & 19.830 \\
\hline PMF029-S & Pu239 & bare & 1.0029900 & $3.16512 \mathrm{D}-03$ & $1.49526 \mathrm{D}+00$ & $1.95823 \mathrm{D}+00$ & 2.060 \\
\hline PMFO29-D & Pu239 & bare & $\odot .9921650$ & $3.15486 \mathrm{D}-03$ & 1. $49189 \mathrm{D}+\odot \odot$ & $1.95770 \mathrm{D}+\odot \odot$ & 2.030 \\
\hline PMF030-S & Pu239 & Graphite & 1.0035800 & $9.34960 \mathrm{D}-\odot 3$ & $1.3817 \odot \mathrm{D}+\odot \odot$ & $1.83862 \mathrm{D}+0 \odot$ & 3.360 \\
\hline PMF030-D & Pu239 & Graphite & 1.0058000 & $9.43656 \mathrm{D}-\odot 3$ & $1.37406 \mathrm{D}+00$ & $1.83497 \mathrm{D}+0 \odot$ & 2.730 \\
\hline PMF031-S & Pu239 & Poly & .0065400 & $4.96984 \mathrm{D}+0 \odot$ & $1.15040 \mathrm{D}+\odot \odot$ & $1.62461 \mathrm{D}+\odot \odot$ & 6.360 \\
\hline PMF031-D & Pu239 & Poly & 1.0076400 & $4.85015 \mathrm{D}+0 \odot$ & $1.15 \odot 43 \mathrm{D}+00$ & $1.61639 \mathrm{D}+00$ & 7.950 \\
\hline PMF032-S & Pu239 & Steel & $\odot .998265 \odot$ & $8.47321 \mathrm{D}-03$ & $1.38710 \mathrm{D}+\odot \odot$ & $1.84099 \mathrm{D}+\odot \odot$ & 7.160 \\
\hline PMF032-D & Pu239 & Steel & 1.0048100 & $8.63256 \mathrm{D}-\odot 3$ & $1.36047 \mathrm{D}+00$ & $1.81719 \mathrm{D}+0 \odot$ & 5.620 \\
\hline PMF033 & Pu239 & ZPPR-21 & 1.0006200 & $3.03494 \mathrm{D}+00$ & $6.58848 \mathrm{D}-01$ & $1.18821 \mathrm{D}+0 \odot$ & 126.910 \\
\hline PMF035 & Pu239 & lead & ๑.9984980 & $7.14222 \mathrm{D}-03$ & 1. $41389 \mathrm{D}+\odot \odot$ & $1.87403 \mathrm{D}+\odot \odot$ & 4.340 \\
\hline PMF036 & Pu239 & Poly & 1.0051600 & $8.53074 \mathrm{D}-01$ & $1.26576 \mathrm{D}+00$ & $1.72961 \mathrm{D}+0 \odot$ & 4.920 \\
\hline PMF039 & Pu239 & Duraluminiun & $\odot .9938870$ & $9.01953 D-03$ & $1.33798 \mathrm{D}+00$ & $1.80366 \mathrm{D}+0 \odot$ & 5.840 \\
\hline PMF०40 & Pu239 & Copper & 1.0009600 & $5.99575 \mathrm{D}-\odot 3$ & $1.31601 \mathrm{D}+00$ & $1.80202 \mathrm{D}+\odot \odot$ & 3.620 \\
\hline PMF041 & Pu239 & D38 & 1.0079200 & $8.54537 D-02$ & $1.57408 \mathrm{D}+\odot \odot$ & $2 . \odot 5025 \mathrm{D}+\odot \odot$ & 18.890 \\
\hline & & & & & & & \\
\hline & & & 1.0001580 & $+/-$ & & Total & 685.900 \\
\hline & & & & & & & \\
\hline & & Highest & 1.0109900 & $\odot .010 \varepsilon$ & 8320 (from A & (erage) & \\
\hline
\end{tabular}

Frequency Distribution for Expected $\mathrm{K}$ Values from $\odot . \odot$ to 2.0

( 0.005 bin width sums)

\begin{tabular}{|c|c|c|}
\hline Expect & $\mathrm{K}$ Range & Occurrences \\
\hline 0.980 & 0.985 & $1 \times$ \\
\hline 0.985 & 0.990 & $\odot$ \\
\hline 0.996 & 0.995 & $9 \mathrm{XXXXXXXXX}$ \\
\hline
\end{tabular}




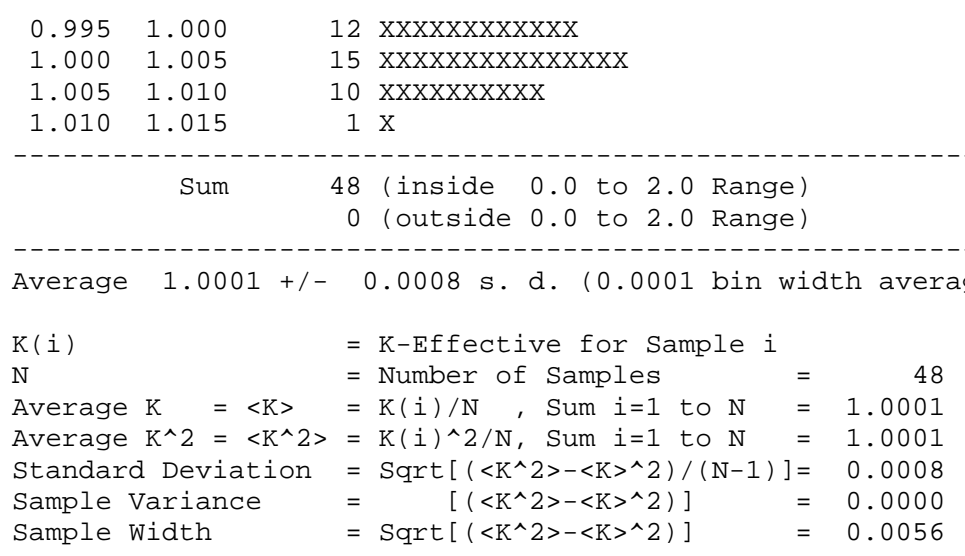

As $\mathrm{N}$ Approaches Infinity Standard Deviation Approaches Zero.

As $\mathrm{N}$ Approaches Infinity Sample width Approaches a Constant.

Confidence Limits

(Occurrences out to $+/-10$ times Sample Width)

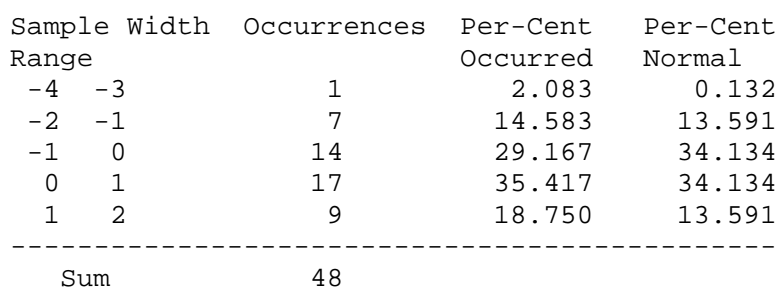




\section{Pu Midi (36 Assemblies)}

Criticality Calculation Editor (CRITEDIT 2002-1)

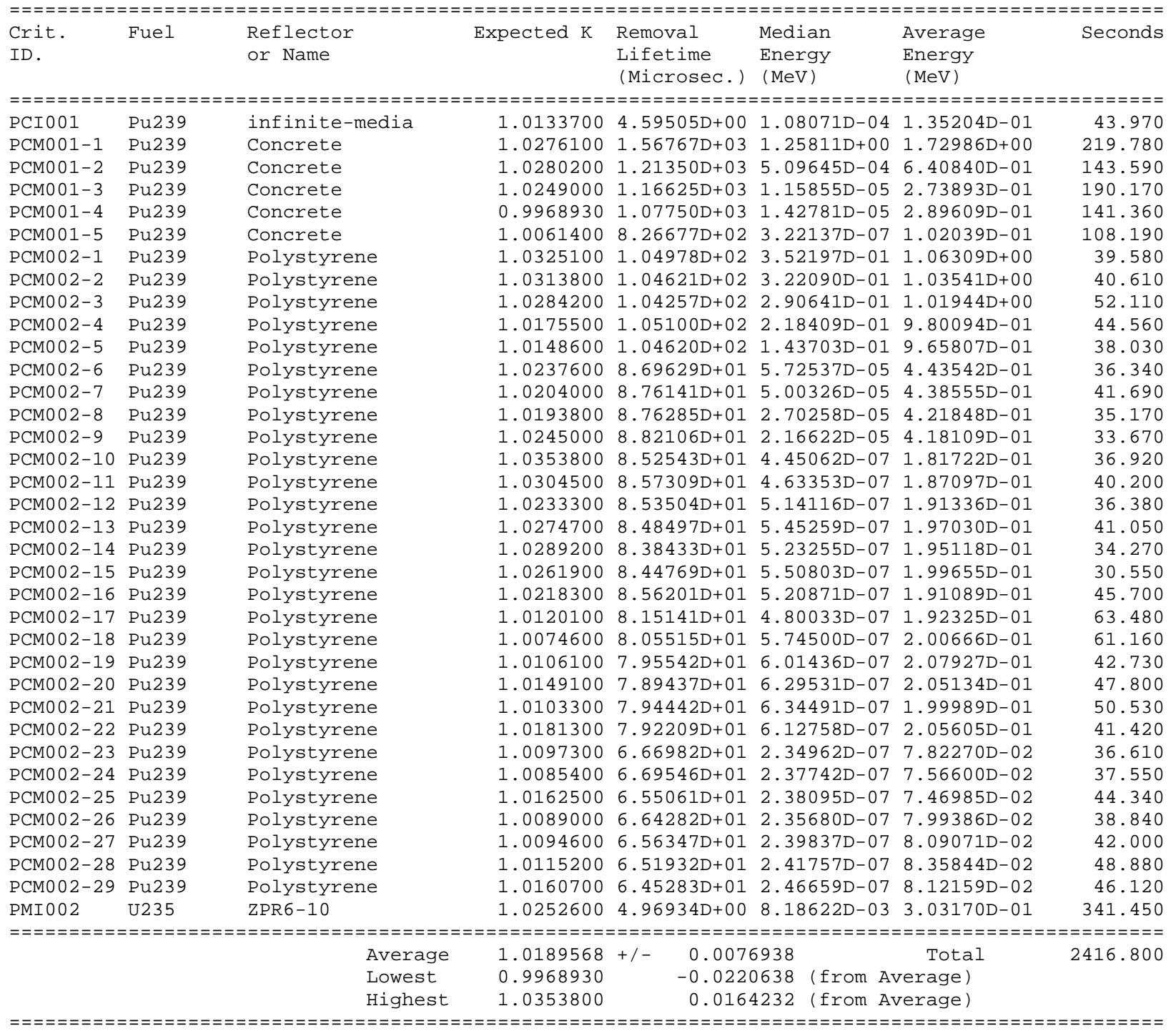

Frequency Distribution for Expected $\mathrm{K}$ Values from $\odot . \odot$ to 2.0 $(0.005$ bin width sums)

\begin{tabular}{|c|c|c|}
\hline Expecte & d K Range & Occurrences \\
\hline 0.995 & 1.000 & $1 x$ \\
\hline 1.000 & 1.005 & $\odot$ \\
\hline 1.005 & 1.010 & $6 \mathrm{XXXXXX}$ \\
\hline 1.010 & 1.015 & $7 \times X X X X X X$ \\
\hline 1.015 & 1.020 & $5 \times X X X X$ \\
\hline 1.020 & 1.025 & $6 \mathrm{XXXXXX}$ \\
\hline 1.025 & 1.030 & $7 \mathrm{XXXXXXX}$ \\
\hline 1.030 & 1.035 & $3 \times X X$ \\
\hline 1.035 & 1.040 & $1 \times$ \\
\hline & Sum & $\begin{array}{r}36 \text { (inside } \odot . \odot \text { to } 2 . \odot \text { Range) } \\
\odot \text { (outside } \odot . \odot \text { to } 2 . \odot \text { Range) }\end{array}$ \\
\hline
\end{tabular}

Average $1.0189+/-0.0015 \mathrm{s.d} .(0.0001$ bin width average) 


\begin{tabular}{|c|c|c|}
\hline $\mathrm{K}(\mathrm{i})$ & $=\mathrm{K}$-Effective for Sample $i$ & \\
\hline $\mathrm{N}$ & $=$ Number of Samples & 36 \\
\hline Average $\mathrm{K}$ & $=K(i) / N$, sum $i=1$ to $N$ & 1.0189 \\
\hline Average $\mathrm{K}^{\wedge} 2=\left\langle\mathrm{K}^{\wedge} 2\right\rangle$ & $=K(i)^{\wedge} 2 / N$, sum $i=1$ to $N$ & 1.0382 \\
\hline Standard Deviation & $=\operatorname{sqrt}\left[\left(<\mathrm{K}^{\wedge} 2>-<\mathrm{K}>\wedge 2\right) /(\mathrm{N}-1)\right]$ & 0.0015 \\
\hline Sample Variance & $=\quad\left[\left(<K^{\wedge} 2>-<K>\wedge 2\right)\right]$ & 0.0001 \\
\hline Sample Width & $=\operatorname{sqrt}\left[\left(<K^{\wedge} 2>-<K>\wedge 2\right)\right]$ & 0.0089 \\
\hline
\end{tabular}

As N Approaches Infinity Standard Deviation Approaches Zero.

As N Approaches Infinity Sample Width Approaches a Constant.

Confidence Limits

(Occurrences out to +/- 10 times Sample Width)

\begin{tabular}{|c|c|c|c|c|}
\hline \multirow{2}{*}{\multicolumn{2}{|c|}{$\begin{array}{l}\text { Sample Widtr } \\
\text { Range }\end{array}$}} & Occurrences & Per-Cent & Per-Cent \\
\hline & & & Occurred & Normal \\
\hline & -2 & 1 & 2.778 & 2.140 \\
\hline$-2-$ & -1 & 6 & 16.667 & 13.591 \\
\hline-1 & 0 & 11 & 30.556 & 34.134 \\
\hline 0 & 1 & 11 & 30.556 & 34.134 \\
\hline 1 & 2 & 7 & 19.444 & 13.591 \\
\hline Sum & & 36 & & \\
\hline
\end{tabular}




\section{Pu Slow (247 Assemblies)}

Criticality Calculation Editor (CRITEDIT 2002-1)

\begin{tabular}{|c|c|c|c|c|c|c|c|}
\hline $\begin{array}{l}\text { Crit. } \\
\text { ID. }\end{array}$ & Fuel & $\begin{array}{l}\text { Reflector } \\
\text { or Name }\end{array}$ & Expected $\mathrm{K}$ & $\begin{array}{l}\text { Removal } \\
\text { Lifetime } \\
\text { (Microsec.) }\end{array}$ & $\begin{array}{l}\text { Median } \\
\text { Energy } \\
(\text { MeV) }\end{array}$ & $\begin{array}{l}\text { Average } \\
\text { Energy } \\
(\mathrm{MeV})\end{array}$ & Seconds \\
\hline
\end{tabular}

PST001-1 Pu239 PSTO01-2 Pu239 PSTO01-3 Pu239 PSTO01-4 PU239 PST001-5 Pu239 PST001- 6 Pu239 PST001-T1 Pu239 PSTO01-T2 PU239 PST001-T3 PU239 PST001-T4 Pu239 PST001-T5 PU239 PST001-T6 Pu239 PST002-1 Pu239 PSTOO2-2 PU239 PSTOO2-3 Pu239 PSTO०2-4 Pu239 PSTO02-5 PU239 PST002-6 Pu239 PSTOO2-7 PU239 PSTO03-1 Pu239 PSTO03-2 PU239 PSTOO3-3 PU239 PSTOO3-4 PU239 PSTO03-5 PU239 PSTO03-6 Pu239 PSTO03-7 PU239 PST003-8 Pu239 PSTO04-1 PU239 PSTO04-2 Pu239 PSTO04-3 PU239 PSTO04-4 Pu239 PSTO04-5 Pu239 PSTO04-6 PU239 PSTO04-7 Pu239 PSTO04-8 PU239 PSTO04-9 Pu239 PSTO04-10 PU239 PST004-11 Pu239 PSTO04-12 Pu239 PST004-13 Pu239 PSTO05-1 PU239 PSTO05-2 Pu239 PSTO05-3 Pu239 PSTO05-4 Pu239 PSTO05-5 Pu239 PSTO05-6 Pu239 PST005-7 Pu239 PSTO05-8 PU239 PST005-9 Pu239 PST006-1 Pu239 PST006-2 Pu239 PSTO06-3 Pu239 PST007-1 Pu239 PSTO07-2 Pu239 PSTO07-3 Pu239 PSTO07-4 Pu239 PSTO07-5 PU239 PSTO07-6 Pu239 PSTOO7-7 PU239
$30 \mathrm{~cm}-\mathrm{H} 2 \mathrm{O}$

$30 \mathrm{~cm}-\mathrm{H} 2 \mathrm{O}$

$30 \mathrm{~cm}-\mathrm{H} 2 \mathrm{O}$

$30 \mathrm{~cm}-\mathrm{H} 2 \mathrm{O}$

$30 \mathrm{~cm}-\mathrm{H} 2 \mathrm{O}$

$30 \mathrm{~cm}-\mathrm{H} 2 \mathrm{O}$

$30 \mathrm{~cm} \_\mathrm{H} 2 \mathrm{O}$

$30 \mathrm{~cm} \_\mathrm{H} 2 \mathrm{O}$

$30 \mathrm{~cm} \_\mathrm{H} 2 \mathrm{O}$

$30 \mathrm{Cm} \_\mathrm{H} 2 \mathrm{O}$

$30 \mathrm{Cm} \_\mathrm{H} 20$

$30 \mathrm{Cm} \_\mathrm{H} 2 \mathrm{O}$

$30 \mathrm{~cm}-\mathrm{H} 2 \mathrm{O}$

$30 \mathrm{~cm}-\mathrm{H} 2 \mathrm{O}$

$30 \mathrm{~cm}-\mathrm{H} 2 \mathrm{O}$

$30 \mathrm{~cm}-\mathrm{H} 2 \mathrm{O}$

$30 \mathrm{~cm}-\mathrm{H} 2 \mathrm{O}$

$30 \mathrm{~cm}-\mathrm{H} 2 \mathrm{O}$

$30 \mathrm{~cm}-\mathrm{H} 2 \mathrm{O}$

$30 \mathrm{~cm}-\mathrm{H} 2 \mathrm{O}$

$30 \mathrm{~cm}-\mathrm{H} 2 \mathrm{O}$

$30 \mathrm{~cm}-\mathrm{H} 2 \mathrm{O}$

$30 \mathrm{~cm}-\mathrm{H} 2 \mathrm{O}$

$30 \mathrm{~cm}-\mathrm{H} 2 \mathrm{O}$

$30 \mathrm{~cm}-\mathrm{H} 2 \mathrm{O}$

$30 \mathrm{~cm}-\mathrm{H} 2 \mathrm{O}$

$30 \mathrm{~cm}-\mathrm{H} 2 \mathrm{O}$

$30 \mathrm{~cm}-\mathrm{H} 2 \mathrm{O}$

$30 \mathrm{~cm}-\mathrm{H} 2 \mathrm{O}$

$30 \mathrm{~cm}-\mathrm{H} 2 \mathrm{O}$

$30 \mathrm{~cm}-\mathrm{H} 2 \mathrm{O}$

$30 \mathrm{~cm}-\mathrm{H} 2 \mathrm{O}$

$30 \mathrm{~cm}-\mathrm{H} 2 \mathrm{O}$

$30 \mathrm{~cm}-\mathrm{H} 2 \mathrm{O}$

$30 \mathrm{~cm}-\mathrm{H} 2 \mathrm{O}$

$30 \mathrm{~cm}-\mathrm{H} 2 \mathrm{O}$

$30 \mathrm{~cm}-\mathrm{H} 2 \mathrm{O}$

$30 \mathrm{~cm}-\mathrm{H} 2 \mathrm{O}$

$30 \mathrm{~cm}-\mathrm{H} 2 \mathrm{O}$

$30 \mathrm{~cm}-\mathrm{H} 2 \mathrm{O}$

$30 \mathrm{~cm}-\mathrm{H} 2 \mathrm{O}$

$30 \mathrm{~cm}-\mathrm{H} 2 \mathrm{O}$

$30 \mathrm{~cm}-\mathrm{H} 2 \mathrm{O}$

$30 \mathrm{~cm}-\mathrm{H} 2 \mathrm{O}$

$30 \mathrm{~cm}-\mathrm{H} 2 \mathrm{O}$

$30 \mathrm{~cm}-\mathrm{H} 2 \mathrm{O}$

$30 \mathrm{~cm}-\mathrm{H} 2 \mathrm{O}$

$30 \mathrm{~cm}-\mathrm{H} 2 \mathrm{O}$

$30 \mathrm{~cm}-\mathrm{H} 2 \mathrm{O}$

$30 \mathrm{~cm}-\mathrm{H} 2 \mathrm{O}$

$30 \mathrm{~cm}-\mathrm{H} 2 \mathrm{O}$

$30 \mathrm{~cm}-\mathrm{H} 2 \mathrm{O}$

water

Water

water

water

water

water

water
$1.00364008 .70141 D+01$ 6.63961D-08 1.35718D-02

$1.00218008 .55592 \mathrm{D}+01 \quad 7.83061 \mathrm{D}-08$ 1.79951D-02

$1.00556008 .43737 \mathrm{D}+01 \quad 9.34902 \mathrm{D}-08$ 2.20241D-02

$0.99732008 .43773 D+01$ 1.00428D-07 2.36321D-02

$1.00328008 .36691 D+01$ 1.05365D-07 2.43300D-02

$1.002150 \odot 8.06327 D+01$ 1.92867D-07 4.63684D-02

$1.00775008 .64639 D+01 \quad 6.53296 D-08$ 1.27809D-02

$1.00255008 .56140 D+01 \quad 7.74814 D-08$ 1.84745D-02

$1.00177008 .44604 \mathrm{D}+01 \quad 9.04530 \mathrm{D}-08 \quad 2.07796 \mathrm{D}-02$

$\begin{array}{llll}1.0026600 & 8.39496 \mathrm{D}+01 & 9.98510 \mathrm{D}-08 & 2.48472 \mathrm{D}-02\end{array}$

$\begin{array}{llll}1.0023500 & 8.31778 D+01 & 1.06114 D-07 & 2.65342 D-02\end{array}$

1.0040800 8.07706D+01 1.93743D-07 4.91729D-02

$1.00655008 .92837 \mathrm{D}+01$ 5.69545D-08 8.96113D-03

1. 0033700 8.89751D+01 5.76394D-08 9.78381D-03

0.9989440 8.90626D+01 5.99269D-08 1.18325D-02

$1.00644008 .79557 D+01 \quad 6.20943 D-08$ 1.16423D-02

$1.00981008 .68181 D+01 \quad 6.41582 D-08$ 1.24360D-02

$1.00336008 .70492 D+01 \quad 6.78370 D-08$ 1.45432D-02

$0.99348908 .79486 \mathrm{D}+01 \quad 7.22954 \mathrm{D}-08$ 1.51837D-02

$1.00579009 .14167 \mathrm{D}+01$ 5.04274D-08 6.66637D-03

$\begin{array}{llll}1.0056400 & 9.19463 D+01 & 5.03280 D-08 & 6.07977 D-03\end{array}$

$1.00643008 .99658 \mathrm{D}+01 \quad 5.18992 \mathrm{D}-08$ 7.29761D-03

$1.00885008 .90124 D+01 \quad 5.25687 D-08$ 8.21094D-03

$1.00088008 .95126 \mathrm{D}+01 \quad 5.34566 \mathrm{D}-08$ 8.60447D-03

$1.00548008 .78204 \mathrm{D}+01$ 5.59582D-08 8.85093D-03

$1.0124100 \quad 9.42147 D+01 \quad 4.98341 D-08 \quad 6.79766 D-03$

1. $00770009.40451 D+01$ 5.08086D-08 7.29928D-03

$\begin{array}{llll}1.0096700 & 9.21845 D+01 & 5.25936 D-08 & 6.27995 D-03\end{array}$

$1.00375009 .18415 D+015.24768 D-08$ 5.50798D-03

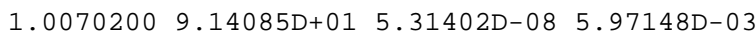

$1.00275009 .11964 \mathrm{D}+01 \quad 5.39248 \mathrm{D}-08$ 6.44561D-03

$1.00460009 .09086 \mathrm{D}+01 \quad 5.31819 \mathrm{D}-08$ 3.62913D-03

$\begin{array}{llll}1.0023800 & 9.06440 D+01 & 5.38002 D-08 & 6.04784 D-03\end{array}$

$1.00978008 .94503 D+01 \quad 5.39275 D-08$ 6.01399D-03

$1.00266008 .95421 D+01 \quad 5.39924 D-08 \quad 6.58711 D-03$

$0.99539608 .93037 D+015.57618 D-08$ 7.14978D-03

$1.00420008 .75492 D+01 \quad 5.78630 D-08$ 8.76564D-03

1.0018000 8.60384D+01 6.11832D-08 8.23580D-03

$0.9997690 \quad 9.05100 D+01 \quad 5.43464 D-08$ 5.30143D-03

$1.0036000 \quad 8.99737 D+01 \quad 5.39301 D-08$ 6.56737D-03

$1.00774009 .01188 \mathrm{D}+01 \quad 4.85887 \mathrm{D}-08$ 6.74518D-03

$1.00444008 .95555 \mathrm{D}+01 \quad 4.90585 \mathrm{D}-08$ 7.40498D-03

$1.00679008 .90080 \mathrm{D}+01$ 4.94846D-08 5.77696D-03

$\begin{array}{llll}1.0103300 & 8.81057 D+01 & 5.13674 D-08 & 6.08379 D-03\end{array}$

$\begin{array}{llll}1.0073400 & 8.71547 D+01 & 5.24348 D-08 & 7.83066 D-03\end{array}$

$\begin{array}{llll}1.0020100 & 8.67420 D+01 & 5.39221 D-08 & 8.70865 D-03\end{array}$

$1.00027008 .70185 \mathrm{D}+01$ 5.49165D-08 7.91925D-03

0.9992760 8.99112D+01 4.92795D-08 6.13732D-03

$1.00226008 .88757 D+01 \quad 4.99756 D-08$ 6.54830D-03

1.0065400 9.06025D+01 4.65578D-08 5.45527D-03

$1.00965008 .97458 \mathrm{D}+01 \quad 4.73511 \mathrm{D}-08$ 5.43289D-03

1.0052300 8.93034D+01 4.91324D-08 4.95568D-03

$\begin{array}{llll}1.0026000 & 8.20177 D+01 & 1.66853 D-07 & 4.03846 D-02\end{array}$

$0.99382608 .27859 D+01 \quad 1.60085 D-07 \quad 3.98805 D-02$

$1.00105008 .62819 \mathrm{D}+01 \quad 7.83668 \mathrm{D}-08$ 1.87889D-02

$1.00183008 .59803 \mathrm{D}+018.14486 \mathrm{D}-08$ 1.95733D-02

$1.00248008 .59103 \mathrm{D}+01$ 7.94657D-08 $1.79576 \mathrm{D}-02$

$\odot .99346708 .68047 D+01 \quad 7.97150 D-08$ 1.86019D-02

$0.98531108 .80490 D+01$ 8.05309D-08 2.05319D-02
73.500

46.000

59.380

49.780

59.090

61.140

25.940

70.520

76.580

47.450

74.120

57.670

61.610

44.380

51.500

68.880

76.810

56.980

52.220

80.270

52.190

70.480

60.720

61.140

71.770

66.980

64.720

52.520

73.140

58.770

64.620

57.450

59.330

71.270

61.050

55.110

50.670

66.470

48.620

72.980

53.950

60.020

42.560

64.810

85.640

93.450

65.970

64.330

60.980

49.780

51.640

44.550

54.390

55.620

55.890

55.420

72.910

72.030

53.300 
PST007-8 Pu239 PST008-1 Pu239 PST008-2 Pu239 PST008-3 Pu239 PST008-4 Pu239 PST008-5 Pu239 PST008-6 Pu239 PST008-7 Pu239 PST008-8 Pu239 PST008-9 Pu239 PST008-10 Pu239 PST008-11 Pu239 PST008-12 Pu239 PST008-13 Pu239 PST008-14 Pu239 PST008-15 Pu239 PST008-16 Pu239 PST008-17 Pu239 PST008-18 Pu239 PST008-19 Pu239 PST008-20 Pu239 PST008-21 Pu239 PST008-22 Pu239 PST008-23 Pu239 PST008-24 Pu239 PST008-25 Pu239 PST008-26 Pu239 PST008-27 Pu239 PST008-28 Pu239 PST008-29 Pu239 PST009-1 Pu239 PST009-1a Pu239 PSTO09-2 Pu239 PST009-2a Pu239 PSTO09-3 Pu239 PSTO09-3a Pu239 Pst010-1 Pu239 Pst010-2 Pu239 Pst010-3 Pu239 Pst010-4 Pu239 Pst010-5 Pu239 Pst010-6 Pu239 Pst010-7 Pu239 Pst010-8 Pu239 Pst010-9 Pu239 Pst010-10 Pu239 Pst010-11 Pu239 Pst010-12 Pu239 Pst010-13 Pu239 Pst010-14 Pu239 PST011-1 Pu239 PST011-2 Pu239 PST011-3 Pu239 PST011-4 Pu239 PST011-5 Pu239 PST011-6 Pu239 PST011-7 Pu239 PST011-8 Pu239 PST011-9 Pu239 PST011-10 Pu239 PST011-11 Pu239 PST011-12 Pu239 PST011-13 Pu239 PST020-1 Pu239 PSTO20-2 Pu239 PST020-3 Pu239 PSTO20-4 Pu239 PST020-5 Pu239 PST020-6 Pu239 PST020-7 Pu239 PST020-8 Pu239 water

case-01

case -02

case- 03

case-04

case-05

case- 07

case-08

case-09

case-10

case-11

case-12

case-13

case-14

case-15

case-16

case-17

case-18

case-19

case-20

case- 21

case- 22

case- 23

case-24

case- 25

case- 26

case- 27

case- 28

case-29

case -30

impurities

no-impurities

impurities

no-impurities

impurities

no-impurities

$30 \mathrm{~cm}-\mathrm{H} 2 \mathrm{O}$

$30 \mathrm{~cm}-\mathrm{H} 2 \mathrm{O}$

$30 \mathrm{~cm}-\mathrm{H} 2 \mathrm{O}$

$30 \mathrm{~cm}-\mathrm{H} 2 \mathrm{O}$

$30 \mathrm{~cm}-\mathrm{H} 2 \mathrm{O}$

$30 \mathrm{~cm}-\mathrm{H} 2 \mathrm{O}$

$30 \mathrm{~cm}-\mathrm{H} 2 \mathrm{O}$

$30 \mathrm{~cm}-\mathrm{H} 2 \mathrm{O}$

$30 \mathrm{~cm}-\mathrm{H} 2 \mathrm{O}$

$30 \mathrm{~cm}-\mathrm{H} 2 \mathrm{O}$

$30 \mathrm{~cm}-\mathrm{H} 2 \mathrm{O}$

$30 \mathrm{~cm}-\mathrm{H} 2 \mathrm{O}$

$30 \mathrm{~cm}-\mathrm{H} 2 \mathrm{O}$

$30 \mathrm{~cm}-\mathrm{H} 2 \mathrm{O}$

16-1

$16-2$

$16-3$

$16-4$

$16-5$

18-1

$18-2$

$18-3$

$18-5$

$18-4$

$18-5$

$18-6$

18-7

Water

water

water

water

water

water

water

water $\odot .99858008 .70934 \mathrm{D}+01$ 7.69517D-08 1.62738D-02 $1.01011003 .92148 \mathrm{D}+01$ 5.38864D-08 $7.38649 \mathrm{D}-03$ $1.00607003 .34820 D+01 \quad 6.19512 D-08$ 1.05944D-02 $1.01650003 .26413 D+01 \quad 6.19598 D-08$ 1.12182D-02 1. $01955003.19393 D+01$ 6.42057D-08 1.10866D-02 1. $0212100 \quad 4.08484 \mathrm{D}+01$ 5.16247D-08 8.93216D-03 1. $01304009.48421 D+014.89870 D-08$ 7.05357D-03 $\bullet .99819702 .31786 \mathrm{D}+01 \quad 6.58139 \mathrm{D}-08$ 1.25307D-02 $\begin{array}{lllll}1.0008100 & 1.33481 D+01 & 2.60167 D-07 & 5.30546 D-02\end{array}$ $1.025350 \odot 8.74407 \mathrm{D}+01$ 5.90363D-๑8 9.18861D-๑3 $1.01924008 .96482 \mathrm{D}+01$ 5.42208D-08 8.12608D-03 $1.01531007 .67937 \mathrm{D}+01$ 7.25568D-08 $1.50802 \mathrm{D}-02$ 1. 0265000 8.20953D+01 6.03531D-08 1.00713D-02 $\odot .995450 \odot 2.98210 \mathrm{D}+01$ 8.00980D-08 1.68951D-02 $1.00942004 .72389 D+01$ 5.87102D-08 1.01639D-02 1.0151400 4.29624D+01 5.45297D-08 8.10917D-०3 $\begin{array}{llll}1.0154600 & 3.27902 \mathrm{D}+01 & 6.10636 \mathrm{D}-08 & 1.09672 \mathrm{D}-02\end{array}$

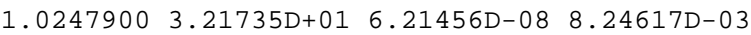
$1.01789004 .54326 D+01$ 5.15111D-08 5.60691D-03

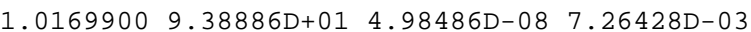
$\odot .99833602 .32511 \mathrm{D}+01$ 6.59800D-08 1.12777D-02 $\odot .99224001 .36097 \mathrm{D}+01 \quad 2.54680 \mathrm{D}-07 \quad 5.42012 \mathrm{D}-02$

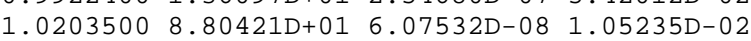

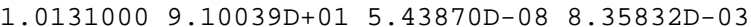
$\begin{array}{lll}1.02294007 .71697 D+01 & 7.38258 D-08 & 1.44012 D-02\end{array}$ $1.01355008 .41255 D+01$ 6.19145D-08 1.19719D-02 $\begin{array}{lllll}1.0050400 & 2.70331 D+01 & 1.03683 D-07 & 2.32181 D-02\end{array}$ $\odot .99964002 .92404 \mathrm{D}+01$ 8.25674D-08 $1.87044 \mathrm{D}-02$ $1.01060004 .64511 D+01$ 6.18635D-08 1.10876D-02 1.0095900 4.71502D+01 5.94385D-08 1.09101D-02 $1.02968007 .46946 \mathrm{D}+01$ 4.57803D-08 2.16496D-03 $1.02891007 .41061 \mathrm{D}+01$ 4.59218D-08 $1.93276 \mathrm{D}-03$ $1.03522007 .81769 \mathrm{D}+01$ 4.54842D-08 4.22196D-@3 $\begin{array}{llll}1.0354100 & 7.80926 \mathrm{D}+01 & 4.57018 \mathrm{D}-08 & 2.22304 \mathrm{D}-03\end{array}$ $\begin{array}{llll}1.0342300 & 7.86886 D+01 & 4.60324 D-08 & 2.09142 D-03\end{array}$

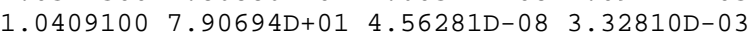
$1.01572008 .54199 D+01$ 7.75892D-08 1.72591D-02 1.0102400 8.69672D+01 6.60308D-08 1.26038D-02 1.0057100 8.82204D+01 5.80501D-08 9.83237D-03 1.0087100 8.40187D+01 5.92470D-08 1.01540D-02 1. $00876008.41276 \mathrm{D}+01$ 5.59988D-08 9.48295D-०3 $1.00858008 .73638 D+01$ 5.53295D-08 8.60001D-०3

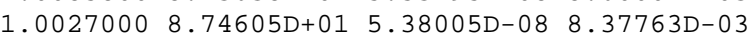

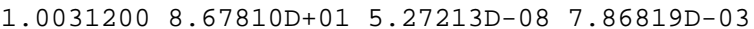

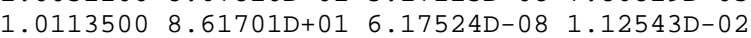
$1.00486008 .74932 \mathrm{D}+01$ 5.60373D-08 8.66968D-๑3 $1.00773008 .68295 \mathrm{D}+01$ 5.60168D-08 9.69776D-०3

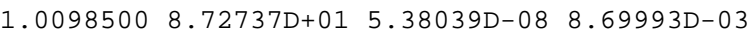

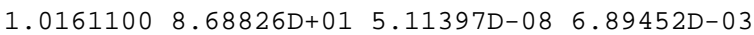
1. $01575008.75824 D+01$ 4.92411D-08 6.79618D-०3

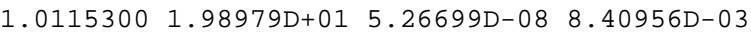

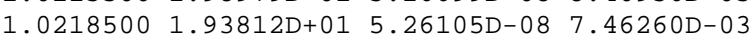
$1.01859001 .82439 D+01$ 5.48256D-08 7.11991D-03

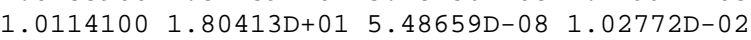
$1.00317001 .53523 D+01 \quad 5.98176 D-08$ 9.24673D-०3 $\odot .99414903 .17520 \mathrm{D}+01$ 4.64298D-08 4.34023D-०3 1. $00407003.07518 \mathrm{D}+01$ 4.74564D-08 4.64952D-03 $\begin{array}{llll}1.0001200 & 3.08449 D+01 & 4.71437 D-08 & 5.49372 D-03\end{array}$ $\begin{array}{llll}1.0124800 & 2.82546 D+01 & 4.88569 D-08 & 5.38291 D-03\end{array}$ $\begin{array}{lllll}1.0006400 & 2.98150 D+01 & 4.78502 D-08 & 4.80366 D-03\end{array}$ $1.01248002 .82546 D+01$ 4.88569D-08 5.38291D-03

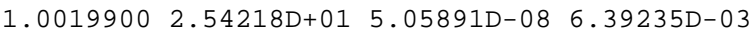

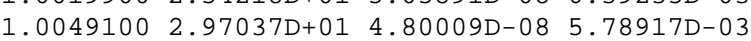
$1.00598008 .69384 \mathrm{D}+01$ 5.41365D-08 8.68941D-03

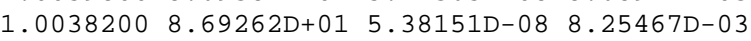
0.9979460 8.95118D+01 4.98461D-08 5.57462D-03 $\bullet .99771708 .55430 \mathrm{D}+01$ 6.00०42D-08 9.76249D-03 $\begin{array}{llll}1.0034400 & 8.13342 D+01 & 6.12871 D-08 & 1.12720 D-02\end{array}$ $1.00123008 .65340 \mathrm{D}+01$ 5.15142D-08 7.78891D-03 $\odot .99909606 .29384 D+01$ 7.94955D-08 1.51324D-02 $\odot .99723706 .54759 D+01$ 6.00789D-08 1.10405D-02
54.450 57.660 54.300 61.410 62.910 53.950

115.670

51.750

35.860

53.470

68.390

72.980

52.910

35.970

51.730

45.000

28.550

25.110

41.220

57.670

33.080

44.410

64.690

45.110

52.800

59.660

36.360

34.410

42.610

44.220

172.270

24.420

179.480

26.330

174.690

25.610

196.060

203.190

268.030

206.980

209.390

211.620

217.450

214.020

205.050

209.910

207.980

210.440

217.980

221.340

20.000

15.420

18.050

22.690

17.580

16.780

23.450

30.110

25.380

31.230

26.410

27.250

22.280

40.470

45.420

44.500

46.720

43.660

31.060

37.030

41.280 
PST020-9 Pu239 PST020-10 Pu239 PST020-11 Pu239 PST020-12 Pu239 PST020-13 Pu239 PST020-14 Pu239 PST020-15 Pu239 PST021-1 Pu239 PST021-2 Pu239 PST021-3 Pu239 PST021-4 Pu239 PST021-5 Pu239 PST021-6 Pu239 PST021-7 Pu239 PST021-8 Pu239 PST021-9 Pu239 PST021-10 Pu239 PST022-D1 Pu239 PST022-D2 Pu239 PST022-D3 Pu239 PST022-D4 Pu239 PST022 - D5 Pu239 PST022-D6 Pu239 PST022 - D7 Pu239 PST022-D8 Pu239 PST022-D9 Pu239 PST022-D10PU239 PST022-D11Pu239 PSTO22 - D12PU239 PST022-D13PU239 PST022-D14PU239 PST022-D15Pu239 PST022-D16Pu239 PST022-D17Pu239 PST022-S1 Pu239 PST022-S2 Pu239 PST022-S3 Pu239 PST022-S4 Pu239 PST022-S5 Pu239 PST022-S6 Pu239 PST022-S7 Pu239 PST022-S8 Pu239 PSTO22-S9 Pu239 PST022-S10PU239 PST022-S11PU239 PST022-S12PU239 PST022-S13PU239 PST022-S14PU239 PST022-S15Pu239 PST022-S16Pu239 PST022-S17Pu239 PST028-D1 Pu239 PST028-D2 Pu239 PST028-D3 Pu239 PST028-D4 Pu239 PST028-D5 Pu239 PST028-D6 Pu239 PST028-D7 Pu239 PST028-D8 Pu239 PST028-D9 Pu239 PST028-D10PU239 PST028-D11Pu239 PST028-D12Pu239 PST028-D13Pu239 PST028-D14PU239 PST028-D15Pu239 PST029-D1 Pu239 PST029-D2 Pu239 PST029-D3 Pu239 PST029-D4 Pu239 PST029-D5 Pu239
Water

water

water

water

water

Water

Water

$\mathrm{H} 2 \mathrm{O}$

$\mathrm{H} 2 \mathrm{O}$

$\mathrm{H} 2 \mathrm{O}$

$\mathrm{H} 2 \mathrm{O}$

$\mathrm{H} 2 \mathrm{O}$

$\mathrm{H} 2 \mathrm{O}$

$\mathrm{H} 2 \mathrm{O}$

$\mathrm{H} 2 \mathrm{O}$

$\mathrm{H} 2 \mathrm{O}$

$\mathrm{H} 2 \mathrm{O}$

soln-H2O

Soln-H2O

Soln-H2O

soln-H2O

Soln-H2O

Soln-H2O

Soln-H2O

Soln-H2O

Soln-H2O

soln- 20

soln-H2O

soln-H2O

soln-H2O

soln-H2O

soln-H2O

soln-H2O

soln-H2O

soln-H2O

Soln-H2O

Soln-H2O

soln- $\mathrm{H} 2 \mathrm{O}$

Soln-H2O

Soln-H2O

Soln-H2O

Soln-H2O

Soln-H2O

soln-H2O

soln-H2O

soln-H2O

soln-H2O

soln-H2O

soln-H2O

soln-H2O

soln-H2O

solution_1

solution_2

solution_3

solution_4

solution_5

solution_6

sol_1+h20

sol8_2+h20

soln_3+H2O

sol_1

sol_1

sol_1

sol_1

sol_1

sol_1

$\operatorname{Exp}-160$

$\operatorname{Exp}-166$

Exp-170

$\operatorname{Exp}-172$

Exp-174 $\begin{array}{llll}1.0064200 & 8.64744 D+01 & 5.30611 D-08 & 6.93972 D-03\end{array}$ $0.99757608 .92241 D+01 \quad 4.98408 D-08$ 6.24663D-03 $1.00062008 .50818 \mathrm{D}+01$ 5.92657D-08 1.08072D-02 $1.00311008 .12761 D+016.15030 D-08$ 1.05392D-02 $\odot .99544006 .55851 D+01 \quad 6.03080 D-08$ 9.41378D-03 $\odot .99494106 .31051 \mathrm{D}+01 \quad 7.84086 \mathrm{D}-08$ 1.41968D- 02 $1.00723008 .85243 D+01 \quad 5.06470 D-08$ 8.02569D-03 $1.00549002 .11626 D+015.41941 D-08$ 9.59373D-03 $\begin{array}{llll}1.0067100 & 2.36814 D+01 & 5.25107 D-08 & 7.51135 D-03\end{array}$ $\odot .99613604 .44286 \mathrm{D}+00 \quad 2.08888 \mathrm{D}-07 \quad 4.13516 \mathrm{D}-02$ $1.0047400 \quad 8.57281 \mathrm{D}+01 \quad 4.71134 \mathrm{D}-08$ 5.25006D-03 $\begin{array}{llll}1.0025300 & 9.02925 D+01 & 4.62872 D-08 & 4.77120 D-03\end{array}$ $1.01045008 .32656 \mathrm{D}+01 \quad 5.63881 \mathrm{D}-08$ 8.60994D-03 $1.00499001 .76480 \mathrm{D}+01 \quad 5.45078 \mathrm{D}-08$ 9.67924D-03 $\begin{array}{llll}1.0027500 & 3.40823 D+00 & 2.07870 D-07 & 3.96676 D-02\end{array}$ $1.00670001 .66946 \mathrm{D}+01 \quad 5.58643 \mathrm{D}-08$ 8.32822D-03 1. $0105700 \quad 9.45449 D+01 \quad 4.54664 D-08 \quad 4.82909 D-03$ $0.99367105 .04133 D+01 \quad 1.37905 D-07 \quad 3.19198 D-02$ $\odot .9968600 \quad 5.19926 \mathrm{D}+01 \quad 8.97132 \mathrm{D}-08$ 2.16249D-02 $0.99684305 .79382 \mathrm{D}+01 \quad 6.36378 \mathrm{D}-08$ 1.24222D-02 $0.99081306 .13725 \mathrm{D}+01 \quad 5.90222 \mathrm{D}-08$ 1.13225D-02 $0.99968606 .43406 \mathrm{D}+01 \quad 5.44153 \mathrm{D}-08$ 7.14218D-03 $\begin{array}{llll}1.0027500 & 6.67503 \mathrm{D}+01 & 5.20296 \mathrm{D}-08 & 9.13555 \mathrm{D}-03\end{array}$ $\begin{array}{llll}1.0039300 & 6.95108 \mathrm{D}+01 & 5.07522 \mathrm{D}-08 & 7.64180 \mathrm{D}-03\end{array}$ $1.01196007 .04987 D+01$ 4.94911D-08 8.89734D-03 $1.00739007 .25553 \mathrm{D}+01$ 4.93236D-08 5.99191D-03 $\odot .99561005 .23394 D+01 \quad 1.44368 D-07 \quad 3.50259 D-02$ $0.98913905 .34728 D+01 \quad 1.14719 D-07 \quad 2.77109 D-02$ $\odot .99118505 .46730 \mathrm{D}+01 \quad 8.88465 \mathrm{D}-08$ 2.28791D-02 $0.98907805 .62164 \mathrm{D}+01 \quad 7.77859 \mathrm{D}-08$ 1.96742D-02 $0.99138805 .74605 \mathrm{D}+01 \quad 7.07360 \mathrm{D}-08$ 1.67979D-02 $0.99121005 .99232 D+01 \quad 6.38307 D-08$ 1.39468D-02 $0.99160206 .08298 D+01 \quad 6.05029 D-08$ 1.26432D-02 $0.98951906 .14237 D+01$ 6.04412D-08 $1.26649 D-02$ $0.99486304 .98494 \mathrm{D}+01$ 1.34422D-07 $3.34946 \mathrm{D}-02$ $\odot .98874805 .28289 D+01 \quad 9.03575 D-08 \quad 2.31429 D-02$ $\odot .99862905 .80469 \mathrm{D}+01 \quad 6.45193 \mathrm{D}-08$ 1.33242D-02 $1.00388006 .09766 \mathrm{D}+01 \quad 5.81334 \mathrm{D}-08$ 1.03832D-02 $1.00239006 .46248 \mathrm{D}+01 \quad 5.38086 \mathrm{D}-08$ 8.91588D-03 $\begin{array}{llll}1.0042700 & 6.73095 D+01 & 5.23933 D-08 & 7.81768 D-03\end{array}$ $1.00519006 .86292 D+01 \quad 5.00380 D-08$ 6.29449D-03 $1.00254007 .07975 D+01 \quad 4.93630 D-08$ 6.86068D-03 $1.01058007 .21895 \mathrm{D}+01$ 4.90896D-08 5.91872D-03 $0.98890505 .23811 \mathrm{D}+01 \quad 1.45046 \mathrm{D}-07 \quad 3.63878 \mathrm{D}-02$ $0.98270605 .33933 \mathrm{D}+01$ 1.14134D-07 2.78124D-02 $\odot .98686005 .43487 D+01 \quad 9.05490 D-08$ 2.12743D-02 $0.98876005 .63016 \mathrm{D}+01 \quad 7.73468 \mathrm{D}-08$ 2.04554D-02 $0.98713405 .73964 \mathrm{D}+01 \quad 7.04664 \mathrm{D}-08$ 1.44991D-02 $\odot .98815405 .99711 \mathrm{D}+01 \quad 6.35838 \mathrm{D}-08$ 1.44236D-02 $0.99612306 .07113 D+01 \quad 6.10762 D-08$ 1.20189D-02 $\odot .99068806 .09833 D+01 \quad 5.99717 D-08$ 1.05098D-02 $1.00738006 .75223 D+01 \quad 7.65844 D-081.71160 D-02$ $1.00725006 .85480 \mathrm{D}+01 \quad 7.10950 \mathrm{D}-08$ 1.59550D-02 $1.00812007 .10807 \mathrm{D}+01 \quad 6.44259 \mathrm{D}-08$ 1.13501D-02 $1.00752007 .41287 D+01 \quad 6.04773 D-08$ 1.05988D-02 $1.00900007 .61736 \mathrm{D}+01 \quad 5.77332 \mathrm{D}-08$ 8.42644D-03 1. $01112008.09132 D+01 \quad 5.29547 D-08$ 8.35373D-03 $1.00520007 .65156 D+01 \quad 7.08223 D-08$ 1.26691D-02 $1.00580007 .76898 \mathrm{D}+01 \quad 6.59118 \mathrm{D}-08$ 1.31805D-02 $1.00837007 .99941 D+01$ 6.11531D-08 1.05406D-02 $1.00120007 .65765 D+01 \quad 7.20938 D-08$ 1.45082D-02 $1.00362007 .13549 \mathrm{D}+01$ 7.43471D-08 1.54941D-02 $1.00690006 .87827 \mathrm{D}+01$ 7.17949D-08 $1.44166 \mathrm{D}-02$ $1.01153006 .69046 \mathrm{D}+01 \quad 7.71812 \mathrm{D}-08$ 1.63033D-02 $1.00707006 .71162 \mathrm{D}+01$ 7.58258D-08 $1.60068 \mathrm{D}-02$ $1.00039006 .96061 \mathrm{D}+01$ 7.18600D-08 1.50534D-02 $1.00543007 .18504 \mathrm{D}+01 \quad 5.54695 \mathrm{D}-08$ 8.16433D-03 1. $00808007.42412 \mathrm{D}+01 \quad 5.53139 \mathrm{D}-08$ 8.90919D-03 1.0116000 7.66491D+01 5.59114D-08 7.39551D-03 $\begin{array}{llll}1.0027000 & 7.93690 D+01 & 5.44683 D-08 & 9.99005 D-03\end{array}$ 1. $00278007.88839 D+01 \quad 5.63535 D-08$ 8.98292D-03
27.250

37.920

38.450

37.800

37.560

34.380

38.500

24.120

24.020

19.250

51.730

51.950

51.340

25.660

16.880

24.300

48.110

61.950

67.420

81.690

80.560

94.300

63.310

63.480

65.830

87.170

54.670

87.830

76.590

63.770

50.970

65.340

75.730

82.920

57.160

80.280

66.720

71.550

71.110

73.800

68.020

80.330

73.950

65.270

72.670

77.730

68.080

76.830

88.530

79.500

75.380

46.250

43.720

39.050

47.910

48.120

47.580

42.940

33.330

42.470

36.750

54.830

35.190

54.890

59.980

53.500

34.690

45.080

44.950

40.200

35.970 

PST029-D7 Pu239 PST029-D8 Pu239 PSTO29-D9 Pu239 PST029-D10PU239 PST029-D11Pu239 PST029-D12PU239 PST029-D13Pu239 PST029-D14PU239 PST029-D15Pu239 PST029-D16Pu239 PST029-D17Pu239 PST032-1 U235 PST032-2 U235 PST032-3 U235 PST032- 4 U235 PST032-5 U235 PST032-6 U235 PST032-7 U235 PST032-8 U235 PST032-9 U235 PST032-10 U235 PST032-11 U235 PST032-12 U235 PST032-13 U235 PST032-14 U235 PST032-15 U235 PST032-16 U235 PST032-17 U235 PST032-S1 U235 PST032-S2 U235 PST032-S3 U235 PST032-S4 U235 PST032-S5 U235 PST032-S6 U235 PST032-S7 U235 PST032-S8 U235 PST032-S9 U235 PST032-S10U235 PST032-S11U235 PST032-S13U235 PST032-S14U235 PSTO32-S15U235 PST032-S16U235 PST032 - S17U235
PST029-D6 PU239 PST032-S12U235
Exp-162

Exp-168

Exp-183

Exp-185

Exp-186

Exp-187

Exp-188

Exp-161

Exp-167

Exp-171

Exp-173

Exp-175

water

water

water

water

water

water

water

water

water

water

water

water

water

water

water

water

water

water

water

water

water

water

water

water

water

water

water

water

water

water

water

water

water

water $\begin{array}{llll}1.00076007 .43908 D+01 & 5.39251 D-08 & 7.67834 D-03\end{array}$ $0.99744907 .86318 D+01 \quad 5.33937 D-08 \quad 6.87572 D-03$

$0.99940206 .48614 \mathrm{D}+01$ 5.64845D-08 8.12453D-03 $1.01001006 .73052 \mathrm{D}+01$ 5.58375D-08 8.53985D-03 $1.01038006 .98952 D+01$ 5.54949D-08 7.03004D-03 $1.00583007 .12539 \mathrm{D}+01 \quad 5.55921 \mathrm{D}-08$ 9.35917D-03

$1.00229007 .22531 D+01$ 5.67573D-08 7.64469D-03

$1.00257007 .30716 \mathrm{D}+01$ 5.49937D-08 1.00856D-02

$1.00551007 .55860 D+01 \quad 5.50097 D-08$ 9.04310D-03

$1.00430007 .79576 \mathrm{D}+01 \quad 5.50752 \mathrm{D}-08$ 8.00758D-03

$0.99434207 .98393 \mathrm{D}+01 \quad 5.51549 \mathrm{D}-08$ 9.82702D-03

$\odot .99742607 .98191 \mathrm{D}+01$ 5.50131D-08 8.44821D-03

$0.99550305 .90700 \mathrm{D}+01 \quad 6.58165 \mathrm{D}-08$ 1.18814D-02

$\begin{array}{llll}1.00004006 .03661 D+01 & 6.23623 D-08 & 1.23886 D-02\end{array}$

$0.99701406 .22842 \mathrm{D}+01$ 6.03636D-08 1.24391D-02

$\odot .99736106 .39356 \mathrm{D}+01$ 5.69977D-08 9.69063D-03

$\begin{array}{llll}1.0071500 & 6.54907 D+01 & 5.44275 D-08 & 8.67176 D-03\end{array}$

$1.00744006 .79465 D+01 \quad 5.26719 D-08$ 7.57963D-03

$1.00561006 .94536 \mathrm{D}+01$ 5.11802D-08 6.77923D-03

$1.00632007 .16123 D+01$ 4.99051D-08 6.40035D-03

$1.00837007 .36343 D+01 \quad 4.91179 D-08$ 4.70408D-03

$1.00404007 .58033 \mathrm{D}+014.84876 \mathrm{D}-08$ 7.74543D-03

$1.01056007 .60178 \mathrm{D}+01 \quad 4.77528 \mathrm{D}-08$ 7.30765D-03

$1.00790007 .71711 \mathrm{D}+01$ 4.68652D-08 5.10374D-03

$\begin{array}{llll}1.0004200 & 6.57766 \mathrm{D}+01 & 5.80255 \mathrm{D}-08 & 1.10860 \mathrm{D}-02\end{array}$

$\begin{array}{llll}1.0030400 & 6.78001 \mathrm{D}+01 & 5.58642 \mathrm{D}-08 & 9.41875 \mathrm{D}-03\end{array}$

$\begin{array}{llll}1.0051900 & 6.92131 D+01 & 5.39556 D-08 & 1.02157 D-02\end{array}$

$1.00654007 .18104 \mathrm{D}+01$ 5.16673D-08 7.76815D-03

$1.01180007 .26709 \mathrm{D}+01$ 5.05786D-08 7.01363D-03

$\odot .99036705 .98712 \mathrm{D}+01 \quad 6.67226 \mathrm{D}-08$ 1.31109D-02

$\odot .99873606 .03400 \mathrm{D}+01 \quad 6.28572 \mathrm{D}-08$ 1.04102D-02

$\begin{array}{llll}1.0000800 & 6.23787 D+01 & 6.00730 D-08 & 1.11282 D-02\end{array}$

$1.00171006 .44036 \mathrm{D}+01 \quad 5.68093 \mathrm{D}-08$ 1.11735D-02

$1.00257006 .60942 \mathrm{D}+01$ 5.41125D-08 7.69171D-03

$1.00182006 .88542 D+01$ 5.20457D-08 8.71490D-03

$1.01056006 .90213 D+015.11527 D-08$ 6.84066D-03

$\begin{array}{llll}1.0076400 & 7.19766 \mathrm{D}+01 & 5.01788 \mathrm{D}-08 & 6.22495 \mathrm{D}-03\end{array}$

$1.00591007 .38189 D+01 \quad 4.89500 D-08$ 6.16861D-03

$1.00980007 .49066 \mathrm{D}+01 \quad 4.79783 \mathrm{D}-08$ 5.81477D-03

$1.01174007 .61703 \mathrm{D}+01 \quad 4.74330 \mathrm{D}-08$ 5.87293D-03

$1.01050007 .73918 \mathrm{D}+01 \quad 4.77382 \mathrm{D}-08$ 4.54424D-03

$1.00366006 .57955 \mathrm{D}+01 \quad 5.79300 \mathrm{D}-08$ 1.03540D-02

$0.99641606 .77393 \mathrm{D}+01 \quad 5.64667 \mathrm{D}-08$ 9.00794D-03

$\begin{array}{llll}1.0026300 & 6.96603 \mathrm{D}+01 & 5.35853 \mathrm{D}-08 & 7.83415 \mathrm{D}-03\end{array}$

$1.00623007 .24743 D+01 \quad 5.14117 D-08$ 8.03136D-03

$1.01315007 .25510 D+01$ 4.99467D-08 6.33372D-03

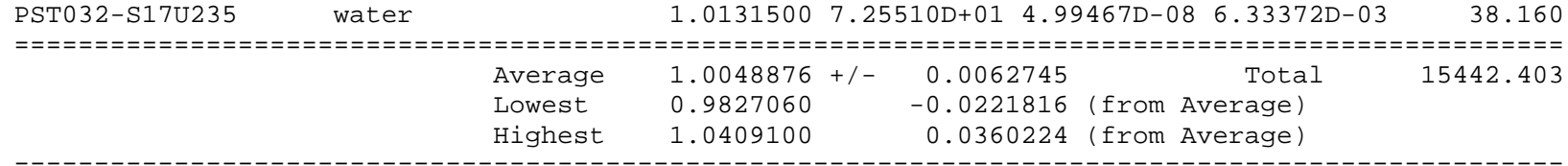

58.420

63.640

52.750

52.330

47.910

42.980

46.590

48.120

41.080

48.620

26.220

36.800

47.450

35.890

51.840

45.030

42.940

35.520

46.310

44.830

37.360

57.410

45.950

46.590

50.270

52.050

37.120

64.230

49.500

38.000

40.950

38.480

47.470

53.560

48.590

51.480

44.440

42.170

47.440

47.080

44.500

53.640

47.660

48.340

49.250

38.160

Frequency Distribution for Expected $\mathrm{K}$ Values from 0.0 to 2.0

( 0.005 bin width sums)

Expected $\mathrm{K}$ Range 0ccurrences

$0.980 \quad 0.985 \quad 1$

$0.985 \quad 0.990 \quad 10 X X X X X X X$

$0.990 \quad 0.995 \quad 16 \quad X X X X X X X X X X X$

$0.995 \quad 1.000 \quad 33 \quad X X X X X X X X X X X X X X X X X X X X X X X$

$1.000 \quad 1.005$

1.0051 .010

$1.010 \quad 1.015$

$1.015 \quad 1.020$

$1.020 \quad 1.025$

$1.025 \quad 1.030$

$1.030 \quad 1.035$

$1.035 \quad 1.040$

$1.040 \quad 1.045$

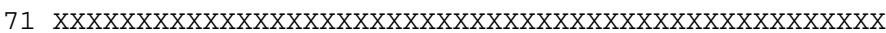

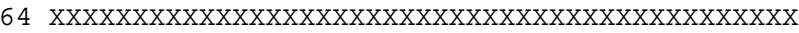

$27 \quad X X X X X X X X X X X X X X X X X X X$

12 XXXXXXXX

$5 \mathrm{XXX}$

$4 \mathrm{XX}$

1

$2 x$

1

Sum

247 (inside $\odot . \odot$ to $2 . \odot$ Range) 
$\odot$ (outside $\odot . \odot$ to $2 . \odot$ Range)

Average 1.0048+/_-

Average $1.0048+/-0.0006 \mathrm{~s} . \mathrm{d} .(0.0001$ bin width average)

$\mathrm{K}(\mathrm{i}) \quad=\mathrm{K}$-Effective for Sample $i$

$\mathrm{N} \quad=$ Number of Samples $=247$

Average $\mathrm{K}=\langle\mathrm{K}\rangle=\mathrm{K}(\mathrm{i}) / \mathrm{N}$, Sum $\mathrm{i}=1$ to $\mathrm{N}=1.0048$

Average $K^{\wedge} 2=\left\langle K^{\wedge} 2\right\rangle=K(i)^{\wedge} 2 / N$, Sum $i=1$ to $N=1.0097$

Standard Deviation $=\operatorname{Sqrt}[(\langle\mathrm{K} \wedge 2>-<\mathrm{K}>\wedge 2) /(\mathrm{N}-1)]=0.0006$

Sample Variance $=\left[\left(<K^{\wedge} 2>-<K>\wedge 2\right)\right]=0.0001$

Sample Width $\quad=\operatorname{Sqrt}\left[\left(\left\langle K^{\wedge} 2>-<K>\wedge 2\right)\right]=0.0088\right.$

As $\mathrm{N}$ Approaches Infinity Standard Deviation Approaches Zero.

As $\mathrm{N}$ Approaches Infinity Sample Width Approaches a Constant.

Confidence Limits

(Occurrences out to +/- 10 times Sample Width)

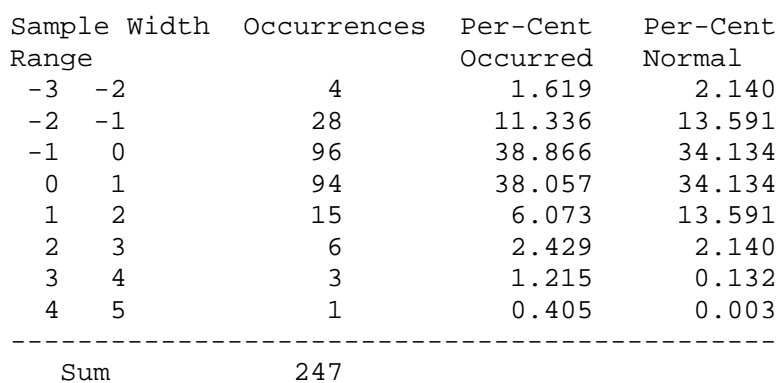




\section{U233 Fast (12 Assemblies)}

\begin{tabular}{|c|c|c|c|c|c|c|c|}
\hline $\begin{array}{l}\text { Crit. } \\
\text { ID. }\end{array}$ & Fuel & $\begin{array}{l}\text { Reflector } \\
\text { or Name }\end{array}$ & Expected $\mathrm{K}$ & $\begin{array}{l}\text { Removal } \\
\text { Lifetime } \\
\text { (Microsec.) }\end{array}$ & $\begin{array}{l}\text { Median } \\
\text { Energy } \\
(\mathrm{MeV})\end{array}$ & $\begin{array}{l}\text { Average } \\
\text { Energy } \\
(\mathrm{MeV})\end{array}$ & Seconds \\
\hline \multicolumn{8}{|c|}{ 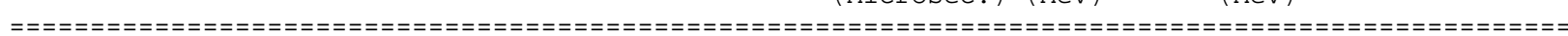 } \\
\hline SFIRO०4 & U233 & Jezebel-23 & 1.0021900 & $3.18656 \mathrm{D}-03$ & $1.31899 \mathrm{D}+0 \odot$ & $1.77804 \mathrm{D}+00$ & 0.660 \\
\hline SFIR๑०7 & U233 & Flattop-23 & 1.0094900 & $7.05817 \mathrm{D}-02$ & 1. $34719 \mathrm{D}+0 \odot$ & $1.86516 \mathrm{D}+0 \odot$ & 4.390 \\
\hline UMF๑०1-1 & U233 & Jezebel & 0.9961170 & $3.14902 \mathrm{D}-03$ & $1.26666 \mathrm{D}+0 \odot$ & $1.75297 \mathrm{D}+0 \odot$ & 0.660 \\
\hline UMF๑०2-1 & U233 & U235 & 0.9942960 & $3.65307 \mathrm{D}-03$ & $1.25475 \mathrm{D}+0 \odot$ & $1.73477 \mathrm{D}+0 \odot$ & 1.000 \\
\hline UMF๑०2-2 & U233 & U235 & 1.0102600 & $4.11639 D-03$ & $1.19558 \mathrm{D}+\odot \odot$ & $1.68293 \mathrm{D}+\odot \odot$ & 1.060 \\
\hline UMF๑०3-1 & U233 & U-nat & 1. $\odot \odot 2520 \odot$ & $5.74215 \mathrm{D}-\odot 3$ & $1.27816 \mathrm{D}+\odot \odot$ & $1.75641 \mathrm{D}+\odot \odot$ & 1.140 \\
\hline UMF००3-2 & U233 & U-nat & 1.0007000 & 1.16169D-02 & $1.26864 \mathrm{D}+0 \odot$ & $1.79267 \mathrm{D}+\odot \odot$ & 1.470 \\
\hline UMF๑०4-1 & U233 & W & 1.0075100 & $6.50212 \mathrm{D}-03$ & $1.12422 \mathrm{D}+0 \odot$ & $1.63480 \mathrm{D}+00$ & 2.690 \\
\hline UMF๑๑4 - 2 & U233 & W & 1. 0106600 & $1.51108 \mathrm{D}-02$ & $1 . \odot 1584 \mathrm{D}+\odot \odot$ & $1.57149 \mathrm{D}+\odot \odot$ & 4.660 \\
\hline UMF๑०5-1 & U233 & $\mathrm{Be}$ & $\odot .9977360$ & $5.97871 \mathrm{D}-03$ & $1.25695 \mathrm{D}+\odot \odot$ & $1.78321 \mathrm{D}+\odot \odot$ & 0.840 \\
\hline UMF๑०5- 2 & U233 & $\mathrm{Be}$ & 0.9940070 & 1. $76 \odot 91 \mathrm{D}-\odot 2$ & $1.21915 \mathrm{D}+\odot \odot$ & $1.80235 \mathrm{D}+\odot \odot$ & 1.060 \\
\hline UMF००6 - 1 & U233 & U-nat & 1.0016700 & $7.12522 D-02$ & $1.30945 \mathrm{D}+0 \odot$ & $1.84086 \mathrm{D}+0 \odot$ & 5.470 \\
\hline \multicolumn{8}{|c|}{ 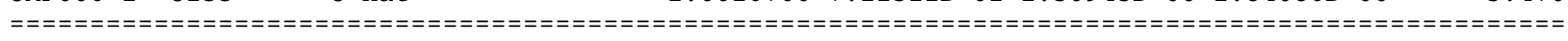 } \\
\hline & & & 1.0022630 & $+/-\quad 0.004$ & 8542 & Total & 25.100 \\
\hline & & & $\odot .9940070$ & & 2560 (from & verage) & \\
\hline & & & 1.0106600 & 0.008 & 3970 (from A & verage) & \\
\hline
\end{tabular}

Frequency Distribution for Expected $K$ Values from 0.0 to 2.0 (๑.๑05 bin width sums)

\begin{tabular}{|c|c|c|c|}
\hline Expecte & d K Range & Occurrences & \\
\hline 0.990 & 0.995 & $2 x X$ & \\
\hline 0.995 & 1.000 & $2 x x$ & \\
\hline 1.000 & 1.005 & $4 X X X X$ & \\
\hline 1.005 & 1.010 & $2 x x$ & \\
\hline 1.010 & 1.015 & $2 x x$ & \\
\hline & Sum & 12 (inside $\odot . \odot$ to $2 . \odot$ Range) & \\
\hline Average & $1.0022+/-$ & 0.0018 s. d. (0.0001 bin wic & th avera \\
\hline$K(i)$ & & $=$ K-Effective for Sample $i$ & \\
\hline $\mathrm{N}$ & & $=$ Number of Samples & 12 \\
\hline Average & $\mathrm{K}=\langle\mathrm{K}\rangle$ & $=K(i) / N$, sum $i=1$ to $N$ & 1.0022 \\
\hline Average & $\mathrm{K}^{\wedge} 2=\left\langle\mathrm{K}^{\wedge} 2\right\rangle$ & $=K(i)^{\wedge} 2 / N$, sum $i=1$ to $N=$ & 1.0044 \\
\hline Standar & d Deviation & $=\operatorname{sqrt}\left[\left(<K^{\wedge} 2>-<K>\wedge 2\right) /(N-1)\right]=$ & $\odot .0018$ \\
\hline Sample & Variance & $=\left[\left(<K^{\wedge} 2>-<K>\wedge 2\right)\right]=$ & 0.0000 \\
\hline Sample & Width & $=\operatorname{Sqrt}\left[\left(<K^{\wedge} 2>-<K>\wedge 2\right)\right]$ & 0.0058 \\
\hline
\end{tabular}

As $\mathrm{N}$ Approaches Infinity Standard Deviation Approaches Zero.

As $\mathrm{N}$ Approaches Infinity Sample Width Approaches a Constant.

Confidence Limits

(Occurrences out to +/- 10 times Sample Width)

Sample Width occurrences Per-Cent Per-Cent Range

\begin{tabular}{rrrrr}
\multicolumn{2}{c}{ Range } & & Occurred & Normal \\
-2 & -1 & 3 & 25.000 & 13.591 \\
-1 & 0 & 4 & 33.333 & 34.134 \\
0 & 1 & 2 & 16.667 & 34.134 \\
1 & 2 & 3 & 25.000 & 13.591 \\
- & Sum & 12 & &
\end{tabular}




\section{U233 Midi (33 Assemblies)}

Criticality Calculation Editor (CRITEDIT 2002-1)

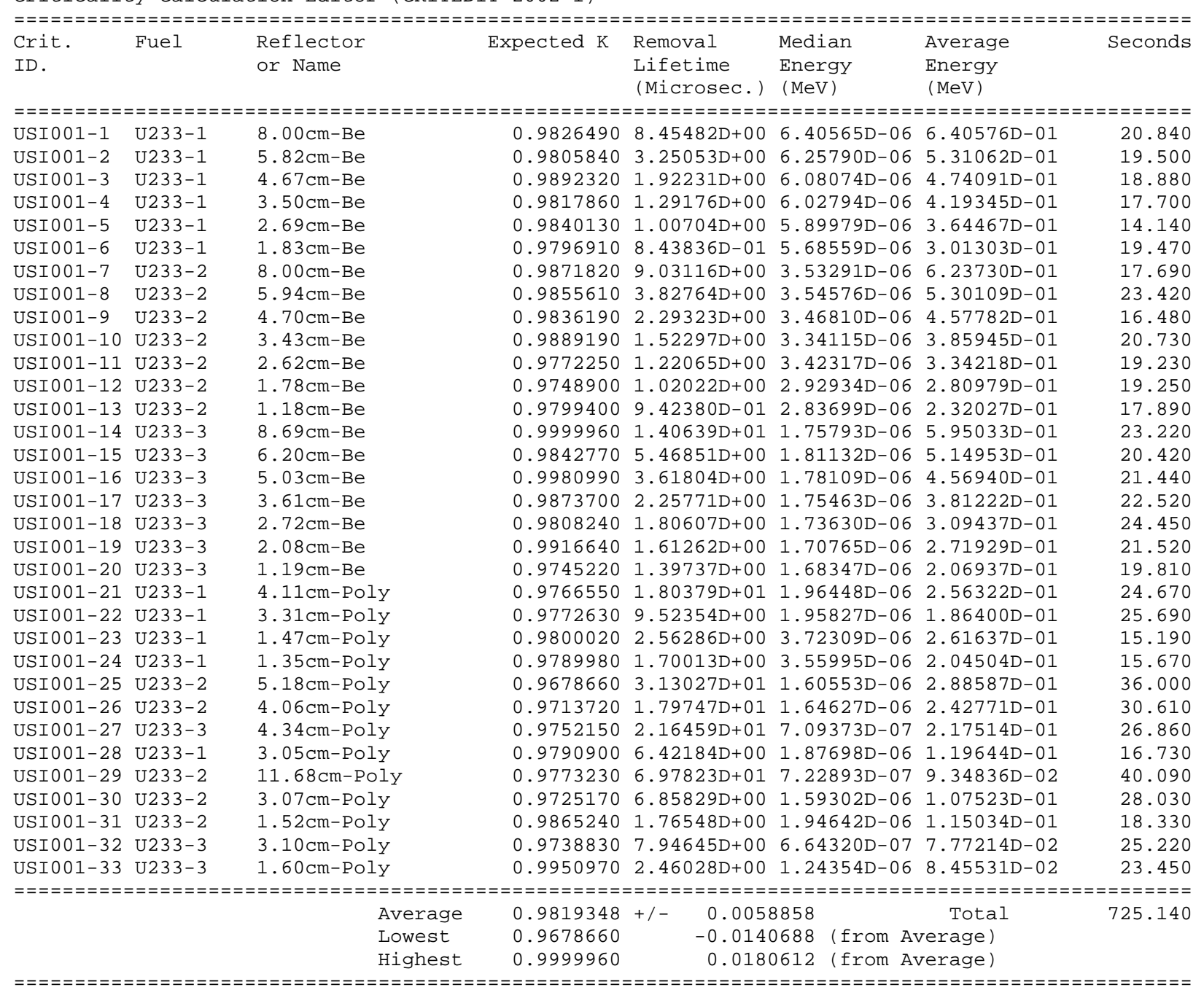

Frequency Distribution for Expected $\mathrm{K}$ Values from 0.0 to 2.0 (0.005 bin width sums)

\begin{tabular}{|c|c|c|}
\hline \multicolumn{2}{|c|}{ Expected $\mathrm{K}$ Range } & Occurrences \\
\hline 0.965 & 0.970 & $1 x$ \\
\hline 0.970 & 0.975 & $5 X X X X X$ \\
\hline 0.975 & 0.980 & $10 X X X X X X X X X X$ \\
\hline 0.980 & 0.985 & 7 XXXXXXX \\
\hline 0.985 & 0.990 & $6 \mathrm{XXXXXX}$ \\
\hline 0.990 & $\odot .995$ & $2 x x$ \\
\hline 0.995 & 1.000 & $2 X X$ \\
\hline
\end{tabular}

33 (inside 0.0 to 2.0 Range)
$\odot$ (outside 0.0 to 2.0 Range)

Average $0.9818+/-0.0013 \mathrm{s.d} \cdot(0.0001$ bin width average)

K(i) $\quad=$ K-Effective for Sample $i$

$\mathrm{N} \quad=$ Number of Samples $=33$

Average $\mathrm{K}=\langle\mathrm{K}\rangle=\mathrm{K}(\mathrm{i}) / \mathrm{N}$, Sum $\mathrm{i}=1$ to $\mathrm{N}=0.9818$

Average $K^{\wedge} 2=\left\langle K^{\wedge} 2\right\rangle=K(i)^{\wedge} 2 / N$, sum $i=1$ to $N=0.9640$

Standard Deviation $=\operatorname{Sqrt}[(\langle\mathrm{K} \wedge 2\rangle-\langle\mathrm{K}\rangle \wedge 2) /(\mathrm{N}-1)]=0.0013$

Sample variance $=[(\langle\mathrm{K} \wedge 2\rangle-\langle\mathrm{K}\rangle \wedge 2)]=0.0001$ 


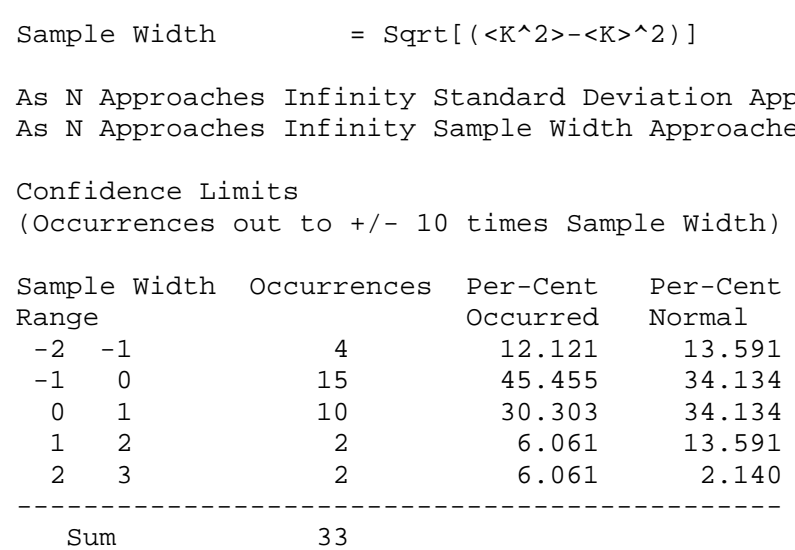




\section{U233 Slow (155 Assemblies)}

Criticality Calculation Editor (CRITEDIT 2002-1)

\begin{tabular}{|c|c|c|c|c|c|c|c|}
\hline $\begin{array}{l}\text { Crit. } \\
\text { ID. }\end{array}$ & Fuel & $\begin{array}{l}\text { Reflector } \\
\text { or Name }\end{array}$ & Expected $\mathrm{K}$ & $\begin{array}{l}\text { Removal } \\
\text { Lifetime } \\
\text { (Microsec.) }\end{array}$ & $\begin{array}{l}\text { Median } \\
\text { Energy } \\
(\mathrm{MeV})\end{array}$ & $\begin{array}{l}\text { Average } \\
\text { Energy } \\
(\mathrm{MeV})\end{array}$ & Seconds \\
\hline
\end{tabular}

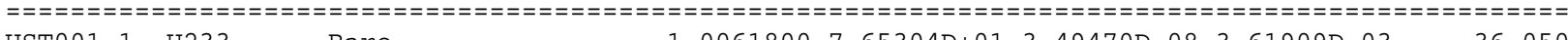

$\begin{array}{llllllllll}\text { UST001-1 U233 } & \text { Bare } & 1.0061800 & 7.65304 D+01 & 3.49470 D-08 & 3.61909 D-03\end{array}$

UST001-2 U233 Bare $\quad 1.00358007 .32001 \mathrm{D}+01 \quad 3.49053 \mathrm{D}-08$ 3.75341D-03

UST001-3 U233 Bare $\quad 1.00330006 .99242 \mathrm{D}+01$ 3.54007D-08 3.99033D-03

UST001-4 U233 Bare $1.00057006 .73201 D+01 \quad 3.53596 \mathrm{D}-08$ 4.03525D-03

UST001-5 U233 Bare $\quad 1.00439006 .54618 D+01 \quad 3.54580 \mathrm{D}-08$ 3.60881D-03

USTO०2-1 U233 paraffin $\quad 1.00603008 .81157 \mathrm{D}+01 \quad 6.37047 \mathrm{D}-08$ 2.67064D-02

USTO०2-2 U233

USTO०2-3 U233

USTO०2-4 U233

USTOO2-5 U233

USTO०2-6 U233

USTOO2-7 U233

USTOO2-8 U233

USTO०2-9 U233

USTO०2-10 U233

USTO०2-11 U233

USTO०2-12 U233

USTO०2-13 U233

USTOO2-14 U233

USTOO2-15 U233

USTOO2-16 U233

USTO०2-17 U233

USTOO3-1 U233

USTOO3-2 U233

USTOO3-3 U233

UST003-4 U233

USTO03-5 U233

USTOO3-6 U233

USTO03-7 U233

USTOO3-8 U233

USTOO3-9 U233

USTOO3-10 U233

USTOO4-1 U233

USTOO4-2 U233

USTOO4-3 U233

USTO०4-4 U233

USTOO4-5 U233

USTOO4-6 U233

USTO०4-7 U233

USTOO4-8 U233

USTO05-1 U233

USTOO5-2 U233

USTO०8-1 U233

USTO09-S1 U233

USTO09-S2 U233

USTOO9-S3 U233

USTO09-S4 U233

UST012-S1 U233

USTO12-S2 U233

UST012-S3 U233

UST012-S4 U233

UST012-S5 U233

USTO12-S6 U233

UST012-S7 U233

UST012-S8 U233

UST012-D1 U233

USTO12-D2 U233

USTO12-D3 U233

UST012-D4 U233

UST012-D5 U233

paraffin

paraffin

paraffin

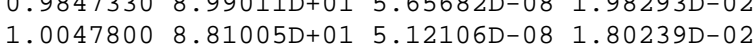

paraffin

paraffin

paraffin

paraffin

paraffin

paraffin

paraffin

paraffin

paraffin

paraffin

paraffin

paraffin

paraffin

Paraffin

Paraffin

Paraffin

Paraffin

Paraffin

Paraffin

Paraffin

Paraffin

Paraffin

Paraffin

paraffin

paraffin

$\odot .99382108 .87959 D+01$ 8.06251D-08 3.65830D-02

$\odot .9889020 \quad 8.78797 D+01$ 1.19190D-07 4.82815D-02

$1.00100008 .87193 \mathrm{D}+01$ 5.77144D-08 2.16388D-02

1. $00696008.80132 D+01$ 4.89827D-08 1.62290D-02

$1.00869008 .94256 \mathrm{D}+01$ 4.60774D-08 $1.25812 \mathrm{D}-02$

1. $01061008.88577 D+01$ 4.42141D-08 1.20360D-02

$0.99469609 .15799 D+01 \quad 4.25992 D-08$ 9.94239D-03

1.0053300 9.05000D+01 4.15825D-08 9.92859D-03

$\odot .98545509 .70549 D+01$ 4.12045D-08 1.08845D-02

$0.9988640 \quad 9.48123 \mathrm{D}+01 \quad 3.95842 \mathrm{D}-08$ 9.59352D-03

$\begin{array}{llll}1.0107200 & 9.23599 D+01 & 3.96841 D-08 & 7.52664 D-03\end{array}$

$\odot .98912201 .02701 \mathrm{D}+02 \quad 3.81808 \mathrm{D}-08$ 8.01231D-03

$\begin{array}{lllll}1.0051000 & 9.96719 D+01 & 3.79858 D-08 & 6.36043 D-03\end{array}$

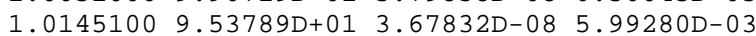

$1.00254008 .45394 \mathrm{D}+01 \quad 8.81972 \mathrm{D}-08$ 3.79068D-02

$1.01861008 .13261 D+01$ 9.59986D-08 4.07437D-02

$1.00306008 .87626 \mathrm{D}+01$ 9.37913D-08 4.06157D-02

$\begin{array}{llll}1.0066200 & 8.11899 D+01 & 2.18518 D-07 & 6.19956 D-02\end{array}$

$1.00773008 .13114 \mathrm{D}+013.14396 \mathrm{D}-07 \quad 7.38415 \mathrm{D}-02$

1.0205100 8.57647D+01 5.60264D-08 2.10009D-02

$\odot .96628007 .13686 \mathrm{D}+01 \quad 4.70563 \mathrm{D}-08$ 1.48044D-02

1.0138400 8.86971D+01 4.33211D-08 1.13640D-02

1.0063900 9.07881D+01 4.10249D-08 9.06018D-03

1. $01301009.62605 D+01 \quad 3.70152 D-08$ 5.32166D-03

$\odot .99923308 .78834 \mathrm{D}+01 \quad 6.42401 \mathrm{D}-08 \quad 2.73426 \mathrm{D}-02$

$1.00553008 .74330 D+01 \quad 5.66869 D-08 \quad 2.28258 D-02$

paraffin

$\odot .99557808 .76845 \mathrm{D}+01 \quad 8.13105 \mathrm{D}-08$ 3.53451D- 02

paraffin

paraffin

paraffin

paraffin

paraffin

$15 \mathrm{~cm}-\mathrm{H} 2 \mathrm{O}$

$15 \mathrm{~cm}-\mathrm{H} 20$

Bare

Soln

Soln

Soln

Soln

$0.98814308 .81291 \mathrm{D}+01$ 1.19829D-07 5. 08838D-02

$0.99726608 .73876 D+01$ 1.02089D-07 4.44018D-02

$1.00500008 .50592 \mathrm{D}+01$ 1.18756D-07 4.97787D-02

$0.9993170 \quad 8.59555 D+01 \quad 9.99215 D-08$ 4.17306D-02

$1.00324008 .60281 D+01 \quad 5.81528 D-08 \quad 2.14943 D-02$

$1.00297009 .58111 D+01$ 4.11239D-08 9.56776D-03

1.0090600 9.98931D+01 3.89906D-08 6.81194D-03

$1.00475001 .00696 \mathrm{D}+02 \quad 3.41519 \mathrm{D}-08$ 4.44015D-03

$\begin{array}{llll}1.0055700 & 9.81153 \mathrm{D}+01 & 3.60363 \mathrm{D}-08 & 3.87423 \mathrm{D}-03\end{array}$

$1.00639001 .01564 \mathrm{D}+02 \quad 3.56764 \mathrm{D}-08$ 4.08596D-03

$\begin{array}{llll}1.0075700 & 1.04748 D+02 & 3.52092 D-08 & 2.86236 D-03\end{array}$

$\begin{array}{llll}1.0069900 & 1.07091 D+02 & 3.55146 \mathrm{D}-08 & 2.78132 \mathrm{D}-03\end{array}$

$\mathrm{H} 2 \mathrm{O}$

$\mathrm{H} 2 \mathrm{O}$

$\mathrm{H} 20$

$\mathrm{H} 2 \mathrm{O}$

$\mathrm{H} 20$

$\mathrm{H} 2 \mathrm{O}$

$\mathrm{H} 2 \mathrm{O}$

$\mathrm{H} 2 \mathrm{O}$

$\mathrm{H} 20$

$\mathrm{H} 2 \mathrm{O}$

$\mathrm{H} 2 \mathrm{O}$

$\mathrm{H} 2 \mathrm{O}$

$\mathrm{H} 2 \mathrm{O}$

$0.99490209 .89995 \mathrm{D}+01 \quad 6.50764 \mathrm{D}-08 \quad 2.61436 \mathrm{D}-02$

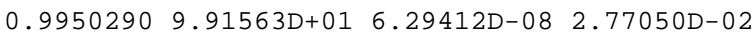

$\begin{array}{llll}1.0076000 & 9.80891 D+01 & 5.93616 D-08 & 2.40316 D-02\end{array}$

1.0029000 9.89591D+01 5.14937D-08 $1.77753 \mathrm{D}-02$

$1.00392001 .00234 \mathrm{D}+02 \quad 4.79871 \mathrm{D}-08$ 1.46051D-02

$1.00445001 .00149 \mathrm{D}+02 \quad 4.59242 \mathrm{D}-08$ 1.34524D-02

$1.00446001 .03570 \mathrm{D}+02 \quad 3.87915 \mathrm{D}-08$ 7.29452D-03

$\odot .99901401 .03601 \mathrm{D}+02$ 3.87645D-08 7.11401D-03

$\odot .99682001 .11888 \mathrm{D}+02 \quad 6.37763 \mathrm{D}-08$ 2.63197D-02

$0.99810301 .11855 \mathrm{D}+02 \quad 6.24557 \mathrm{D}-08 \quad 2.43755 \mathrm{D}-02$

$1.00807001 .10291 \mathrm{D}+02$ 5.87446D-08 2.40594D-02

$\odot .99998001 .11436 D+02 \quad 5.12407 D-08$ 1.84949D-02

$1.00252001 .11887 \mathrm{D}+02$ 4.77181D-08 1.58931D-02

$1.00061001 .11261 D+02$ 4.59539D-08 1.35900D-๑2

40.080

33.300

29.970

33.840

49.860

39.380

49.730

42.860

43.590

36.300

44.030

39.690

43.360

45.880

34.520

40.530

49.330

49.640

46.560

48.770

50.830

75.520

141.060

105.080

120.080

89.970

153.120

105.620

128.660

222.810

135.660

41.750

49.620

43.750

36.700

51.270

57.800

47.200

43.610

39.420

38.980

32.730

35.090

34.000

34.780

35.000

53.420

63.440

55.270

49.020

47.470

56.610

64.200

39.300

66.880

75.940

57.910

71.720

70.050

59.250 
UST012-D7 U233 USTO12-D8 U233 UST013-1 U233 USTO13-2 U233 USTO13-3 U233 USTO13-4 U233 USTO13-5 U233 UST013-6 U233 UST013-7 U233 UST013-8 U233 UST013-9 U233 UST013-10 U233 USTO13-11 U233 UST013-12 U233 UST013-13 U233 UST013-14 U233 UST013-15 U233 UST013-16 U233 UST013-17 U233 UST013-18 U233 USTO13-19 U233 UST013-20 U233 UST013-21 U233 UST015-1 U233 USTO15-2 U233 UST015-3 U233 USTO15-4 U233 UST015-5 U233 UST015-6 U233 UST015-7 U233 USTO15-8 U233 USTO15-9 U233 USTO15-10 U233 UST015-11 U233 USTO15-12 U233 UST015-13 U233 USTO15-14 U233 UST015-15 U233 UST015-16 U233 UST015-17 U233 UST015-18 U233 UST015-19 U233 USTO15-20 U233 UST015-21 U233 UST015-22 U233 USTO15-23 U233 UST015-24 U233 USTO15-25 U233 UST015-26 U233 UST015-27 U233 UST015-28 U233 UST015-29 U233 USTO15-30 U233 USTO15-31 U233 USTO16-1 U233 USTO16-2 U233 USTO16-3 U233 UST016-4 U233 UST016-5 U233 UST016-6 U233 UST016-7 U233 UST016-8 U233 UST016-9 U233 USTO16-10 U233 UST016-11 U233 UST016-12 U233 UST016-13 U233 UST016-14 U233 UST016-15 U233 USTO16-16 U233 UST016-17 U233
$\mathrm{H} 2 \mathrm{O}$

$\mathrm{H} 2 \mathrm{O}$

Solution

Solution

Solution

Solution

Solution

Solution

Solution

Solution

Solution

Solution

Solution

Solution

Solution

Solution

Solution

Solution

Solution

Solution

Solution

Solution

Solution

Be-4, 1

Be $-4,2$

$\mathrm{Be}-4,3$

Be+Poly $-4,3$

Be $-4,4$

Be $-4,5$

Poly-4, 5

Be $-4,6$

Be $-4,7$

Poly-4, 7

Be $-5,1$

Be $-5,2$

Be $-5,3$

Be+Poly-4, 3

Be $-5,4$

Be $-5,5$

Poly-5, 5

$\mathrm{Be}-5,6$

$\mathrm{Be}-5,7$

Be $-6,1$

Be $-6,2$

Be $-6,3$

Be $-6,4$

Be $-6,5$

Poly-6, 5

Be - 7, 3

Be - 7, 4

Be $-7,5$

$\mathrm{Be}-7,6$

$\mathrm{Be}-7,7$

Be $-7,8$

Expt93

Expt94

Expt95

Expt96

Expt97

Expt98

Expt100

Expt101

Expt102

Expt103

Expt104

Expt105

Expt134

Expt135

Expt136

Expt139

Expt140
$1.00794001 .11819 D+02 \quad 3.93294 D-08 \quad 6.71791 D-03$ $1.00005001 .12252 \mathrm{D}+02 \quad 3.92619 \mathrm{D}-08$ 8.42182D-03 $\begin{array}{llll}1.0034900 & 8.19067 D+00 & 6.17036 D-08 & 2.24377 D-02\end{array}$ $1.0014600 \quad 8.14307 \mathrm{D}+00 \quad 6.22809 \mathrm{D}-08$ 2.48095D-02 $\begin{array}{llll}1.0050400 & 8.18382 D+00 & 6.20797 D-08 & 2.18354 D-02\end{array}$ 1. $0028700 \quad 8.18524 \mathrm{D}+00 \quad 6.15808 \mathrm{D}-08 \quad 2.45090 \mathrm{D}-02$ $\begin{array}{llll}1.0027900 & 8.15737 D+00 & 6.16496 \mathrm{D}-08 & 2.30757 \mathrm{D}-02\end{array}$ $\begin{array}{llll}1.0056600 & 8.50239 D+00 & 6.07059 D-08 & 2.24797 D-02\end{array}$ $\begin{array}{llll}1.0064800 & 8.48610 D+00 & 6.05816 D-08 & 2.37839 D-02\end{array}$ $\begin{array}{llll}1.0058000 & 8.48673 D+00 & 6.03595 D-08 & 2.21162 D-02\end{array}$ $\begin{array}{llll}1.0049600 & 8.47732 \mathrm{D}+00 & 6.05386 \mathrm{D}-08 & 2.16763 \mathrm{D}-02\end{array}$ $1.00815008 .53402 \mathrm{D}+00 \quad 6.05829 \mathrm{D}-08$ 2.33550D-02 $1.0001000 \quad 8.45987 \mathrm{D}+00 \quad 5.98851 \mathrm{D}-08 \quad 2.34682 \mathrm{D}-02$ $\odot .9995150 \quad 8.45841 \mathrm{D}+00 \quad 6.06536 \mathrm{D}-08 \quad 2.23408 \mathrm{D}-02$ $0.99612508 .40680 \mathrm{D}+00$ 6.06601D-08 2.24591D-02 $\begin{array}{llll}1.0048900 & 8.49966 D+0 \odot & 6.05815 D-08 & 2.34063 D-02\end{array}$ $\begin{array}{llll}1.0188000 & 1.21703 D+01 & 5.08849 D-08 & 1.69329 D-02\end{array}$ $0.99276201 .44157 \mathrm{D}+01$ 4.71692D-08 1.28432D-02 $\odot .99546601 .50986 \mathrm{D}+01 \quad 4.71401 \mathrm{D}-08$ 1.53410D-02 $0.99771301 .62467 D+01 \quad 4.54354 D-08$ 1.49589D-02 $\odot .99692601 .66554 \mathrm{D}+01$ 4.56244D-08 $1.38412 \mathrm{D}-02$ $1.00318002 .45237 \mathrm{D}+01 \quad 4.10881 \mathrm{D}-08$ 9.83024D-03 $\odot .99708102 .81090 \mathrm{D}+01 \quad 3.99232 \mathrm{D}-08 \quad 9.34636 \mathrm{D}-03$ $0.97804701 .37211 \mathrm{D}+01$ 1.32543D-06 5.96311D-01 $\odot .98288706 .19019 D+00 \quad 1.30502 D-06 \quad 5.12304 D-01$ $0.98439404 .18522 \mathrm{D}+00$ 1.28076D-06 4.41726D-01 $\odot .99013102 .70167 D+01 \quad 2.44955 D-07 \quad 1.98916 D-01$ $0.98328102 .91721 D+00 \quad 1.24269 D-06 \quad 3.70149 D-01$ $\odot .97266702 .40050 \mathrm{D}+00 \quad 1.16768 \mathrm{D}-06 \quad 3.11442 \mathrm{D}-01$ $0.98359301 .15579 D+01 \quad 2.60225 D-07 \quad 5.90542 D-02$ $0.96795402 .07783 \mathrm{D}+00 \quad 1.13325 \mathrm{D}-06 \quad 2.49643 \mathrm{D}-01$ $0.96929301 .90894 \mathrm{D}+0 \odot$ 1.01781D-06 1.83796D-01 $\odot .98624003 .27766 \mathrm{D}+00 \quad 4.87734 \mathrm{D}-07 \quad 6.83759 \mathrm{D}-02$ $\odot .98499201 .80549 D+01 \quad 6.16293 D-07 \quad 6.01735 D-01$ $0.98249508 .35870 D+00 \quad 6.66513 D-07 \quad 5.20114 D-01$ $0.9841900 \quad 5.56253 \mathrm{D}+00 \quad 6.26484 \mathrm{D}-07 \quad 4.55710 \mathrm{D}-01$ $0.9975770 \quad 4.00027 D+01 \quad 1.40853 D-07 \quad 1.83316 D-01$ $\odot .98720503 .91161 D+00 \quad 5.62898 D-07 \quad 3.58862 D-01$ $0.98248903 .22684 \mathrm{D}+00 \quad 5.53958 \mathrm{D}-07 \quad 3.13118 \mathrm{D}-01$ $0.99540901 .58635 \mathrm{D}+01 \quad 1.39838 \mathrm{D}-07 \quad 4.66474 \mathrm{D}-02$ $0.96997502 .70611 D+00 \quad 4.91815 D-07 \quad 2.46428 D-01$

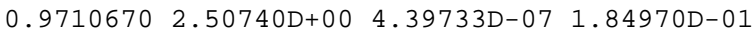
$0.98808603 .85795 \mathrm{D}+01$ 1.47105D-07 6.21720D-01 $\odot .98479301 .69815 \mathrm{D}+01$ 1.54409D-07 5.38212D-01 $\odot .98731501 .06430 D+01$ 1.51299D-07 4.68924D-01 $0.99149607 .31825 \mathrm{D}+00$ 1.37938D-07 3.86528D-01 $\odot .98619705 .97622 D+0 \odot \quad 1.33967 D-07 \quad 3.31555 D-01$ $0.99325803 .06488 D+01 \quad 7.50035 D-08 \quad 3.03240 D-02$ $0.98077103 .49756 \mathrm{D}+01 \quad 7.06088 \mathrm{D}-08$ 5.54016D-01 $0.98789602 .12195 D+01 \quad 6.81673 D-08$ 4.69797D-01 $\odot .98679201 .59481 D+01 \quad 6.81489 D-08$ 4.02006D-01 $0.98587301 .25507 \mathrm{D}+01 \quad 6.54421 \mathrm{D}-08 \quad 3.22598 \mathrm{D}-01$ $0.99227101 .09866 \mathrm{D}+01 \quad 6.35757 \mathrm{D}-08$ 2.62263D-01 $\odot .98903109 .54481 D+00 \quad 6.14255 D-08$ 1.65523D-01 $0.99279501 .88043 \mathrm{D}+03 \quad 9.42526 \mathrm{D}-08 \quad 3.67011 \mathrm{D}-02$ $1.00624001 .85242 \mathrm{D}+03 \quad 9.29520 \mathrm{D}-08$ 3.47946D-02 $\odot .99592401 .84950 D+03 \quad 9.48729 D-08 \quad 3.56561 D-02$ $1.00362001 .83874 D+03 \quad 9.43080 D-08 \quad 3.74361 D-02$ $1.00162001 .83169 D+03 \quad 9.43523 D-08$ 3.46596D-02 $0.99004701 .86329 D+03 \quad 9.42631 D-08$ 3.52343D-02 $\odot .98845701 .86560 \mathrm{D}+03 \quad 9.49275 \mathrm{D}-08$ 3.78593D-02 $\odot .99147401 .84630 \mathrm{D}+03 \quad 9.45282 \mathrm{D}-08 \quad 3.53935 \mathrm{D}-02$ $\odot .99408901 .84370 \mathrm{D}+03 \quad 9.32139 \mathrm{D}-08$ 3.55311D-02 $0.99893901 .83423 D+03 \quad 9.21824 D-08$ 3.63359D-02

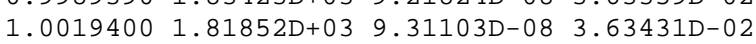
$1.00374001 .81702 \mathrm{D}+03$ 9.34594D-08 3.57538D-02 $\odot .99641201 .83882 D+03 \quad 6.26694 D-08 \quad 2.21383 D-02$ $\begin{array}{llll}1.0148800 & 1.80521 D+03 & 6.29217 D-08 & 2.24845 D-02\end{array}$ $\begin{array}{llll}1.0012600 & 1.83620 D+03 & 6.27152 D-08 & 2.28680 D-02\end{array}$ $\begin{array}{llll}1.0048100 & 1.81463 D+03 & 6.22824 D-08 & 2.55197 D-02\end{array}$ $\odot .98815101 .87042 D+03 \quad 6.25443 D-08 \quad 2.22807 D-02$
26.620

45.120

28.920

27.530

23.720

20.200

26.800

24.970

22.220

22.380

33.440

25.970

26.980

30.360

24.690

26.920

28.440

23.950

27.170

29.220

27.880

44.660

32.310

23.980

22.220

27.390

47.050

29.780

24.090

29.250

16.880

15.390

18.980

18.110

24.060

18.550

39.660

27.230

19.110

29.620

22.950

19.810

28.500

23.120

26.140

21.030

22.910

41.420

30.140

21.360

26.450

22.330

25.060

24.810

119.530

122.050

124.610

132.940

159.300

160.310

120.140

145.440

135.690

162.190

154.250

118.050

124.690

138.060

141.620

115.920

123.200 


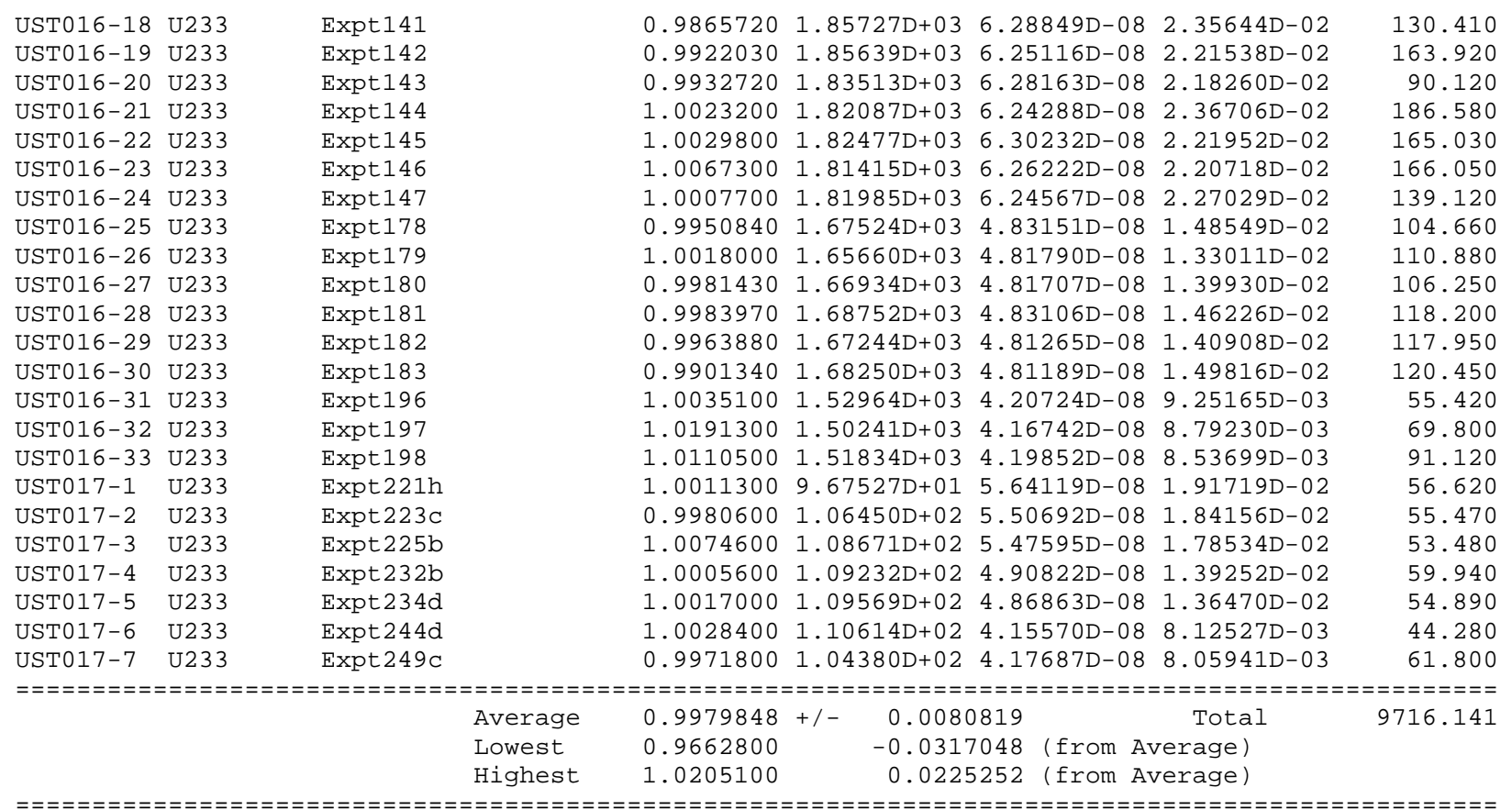

Frequency Distribution for Expected $\mathrm{K}$ Values from 0.0 to 2.0

( 0.005 bin width sums)

\begin{tabular}{|c|c|c|}
\hline \multicolumn{2}{|c|}{ Expected K Range } & Occurrences \\
\hline 0.965 & 0.970 & $4 X X X X$ \\
\hline 0.970 & $\odot .975$ & $2 x X$ \\
\hline$\odot .975$ & $\odot .980$ & $1 \times$ \\
\hline 0.980 & $\odot .985$ & $11 X X X X X X X X X X X$ \\
\hline$\odot .985$ & 0.990 & $17 X X X X X X X X X X X X X X X X X$ \\
\hline$\odot .990$ & 0.995 & $16 \quad X X X X X X X X X X X X X X X X$ \\
\hline$\odot .995$ & $1.00 \odot$ & $26 X X X X X X X X X X X X X X X X X X X X X X X X X X$ \\
\hline 1.000 & 1.005 & 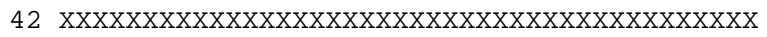 \\
\hline 1.005 & 1.010 & $25 X X X X X X X X X X X X X X X X X X X X X X X X X$ \\
\hline 1.010 & 1.015 & $7 X X X X X X X$ \\
\hline 1.015 & 1.020 & $3 \times X X$ \\
\hline 1.020 & 1.025 & $1 \times$ \\
\hline
\end{tabular}

Sum 155 (inside $\odot . \odot$ to $2 . \odot$ Range)

$\odot$ (outside 0.0 to 2.0 Range)

Average $0.9979+/-0.0008$ s. d. $(0.0001$ bin width average)

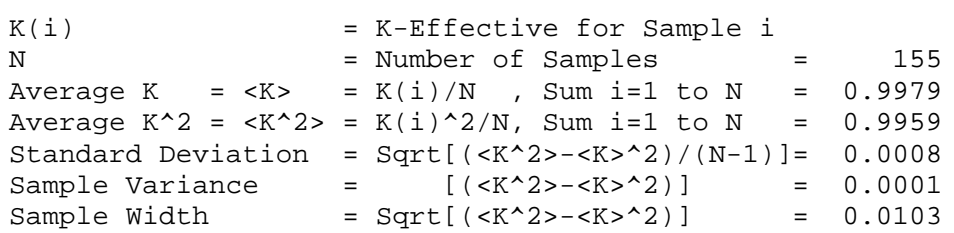

As $\mathrm{N}$ Approaches Infinity Standard Deviation Approaches Zero.

As $\mathrm{N}$ Approaches Infinity Sample Width Approaches a Constant.

Confidence Limits

(Occurrences out to +/- 10 times Sample Width)

Sample Width occurrences Per-Cent Per-Cent Range

$\begin{array}{lr}-4 & -3 \\ -3 & -2 \\ -2 & -1 \\ -1 & 0\end{array}$

1
5
20
39

$\begin{array}{rr}\text { Per-Cent } & \text { Per-Cent } \\ \text { Occurred } & \text { Normal } \\ 0.645 & 0.132 \\ 3.226 & 2.140 \\ 12.903 & 13.591 \\ 25.161 & 34.134\end{array}$




\begin{tabular}{rrrrr}
0 & 1 & 77 & 49.677 & 34.134 \\
1 & 2 & 9 & 5.806 & 13.591 \\
2 & 3 & 4 & 2.581 & 2.140 \\
\hline \multicolumn{2}{c}{ Sum } & 155 & &
\end{tabular}




\section{U235 Fast (199 Assemblies)}

Criticality Calculation Editor (CRITEDIT 2002-1)

\begin{tabular}{|c|c|c|c|c|c|c|c|}
\hline $\begin{array}{l}\text { Crit. } \\
\text { ID. }\end{array}$ & Fuel & $\begin{array}{l}\text { Reflector } \\
\text { or Name }\end{array}$ & Expected $\mathrm{K}$ & $\begin{array}{l}\text { Removal } \\
\text { Lifetime } \\
\text { (Microsec.) }\end{array}$ & $\begin{array}{l}\text { Median } \\
\text { Energy } \\
(\text { MeV) }\end{array}$ & $\begin{array}{l}\text { Average } \\
\text { Energy } \\
(\mathrm{MeV})\end{array}$ & Seconds \\
\hline
\end{tabular}

HMFOO1-1 U235

HMFO01-2 U235

HMFOO2-1 U235

HMFOO2-2 U235

HMFOO2-3 U235

HMFO०2-4 U235

HMFOO2-5 U235

HMFOO2-6 U235

HmfOO3-1 U235

Hmf003-2 U235

HmfO03-3 U235

HmfOO3-4 U235

Hmf003-5 U235

Hmf०03-6 U235

HmfOO3-7 U235

Hmf003-8 U235

Hmfö3-9 U235

Hmf003-10 U235

Hmf003-11 U235

Hmf०03-12 U235

HMF007-1 U235

HMFO०7-2 U235

HMFOO7-3 U235

HMFOO7-4 U235

HMFOO7-5 U235

HMFOO7-6 U235

HMFO०7-7 U235

HMFOO7-8 U235

HMFOO7-9 U235

HMF007-10 U235

HMF007-11 U235

HMFO०7-12 U235

HMFO०7-13 U235

HMFOO7-14 U235

HMFOO7-15 U235

HMF007-16 U235

HMF007-17 U235

HMFOO7-18 U235

HMF007-19 U235

HMFO०7-20 U235

HMF007-21 U235

HMFOO7-22 U235

HMF007-23 U235

HMFOO7-24 U235

HMFO०7-25 U235

HMFOO7-26 U235

HMFOO7-27 U235

HMF007-28 U235

HMFOO7-29 U235

HMFOO7-30 U235

HMF०07-31 U235

HMF007-32 U235

HMFOO7-33 U235

HMFOO7-34 U235

HMFO०7-35 U235

HMFOO7-36 U235

HMFO०7-37 U235

HMF०07-38 U235

HMFO०7-39 U235

HMFO० 7-4९ U235

HMF007-41 U235
Godiva

Godiva

Tuballoy-sphere

Tuballoy-cyl

Tuballoy-box

Tuballoy-box

Tuballoy-box

Tuballoy-box

2 -inches-U

3 -inches $-U$

4 -inches $-U$

5 -inches-U

7 -inches $-U$

8 -inches- $U$

11 - inches - $U$

1.9-inches-WC

2.9-inches-WC

4.5-inches-WC

6.5-inches-WC

8. 0 -inches-Ni

Bare

Poly

Poly

Poly

Poly

Poly

Poly

Poly

Poly

Poly

Poly

Poly

Poly

Poly

Poly

Poly

Poly

Poly

Bare

Poly

Poly

Poly

Poly

Poly

Poly

Poly

Plexi

Plexi

Plexi

Plexi

Plexi

Teflon

Teflon

Teflon

Poly

Poly

Poly

Poly

Poly

Poly

Poly
$1.00429006 .23205 \mathrm{D}-03$ 9.55259D-01 1.51058D+00

$1.00235006 .19500 \mathrm{D}-03$ 9.75280D-01 $1.52076 \mathrm{D}+00$

$\odot .99851707 .65880 \mathrm{D}-02$ 9.44777D-01 $1.61050 \mathrm{D}+00$

$\odot .99759208 .42132 D-02$ 9.07787D-01 1.58847D+0๑

$1.00511008 .87250 \mathrm{D}-02$ 9.09292D-01 $1.59032 \mathrm{D}+00$

$0.99607208 .95613 \mathrm{D}-02$ 8.94650D-01 $1.57932 \mathrm{D}+0 \odot$

$\odot .99525008 .97188 \mathrm{D}-02 \quad 8.83014 \mathrm{D}-01 \quad 1.57476 \mathrm{D}+00$

$0.99925108 .87183 \mathrm{D}-02$ 8.89290D-01 $1.57026 \mathrm{D}+00$

$\odot .994484 \odot 1.48527 \mathrm{D}-02$ 9.29636D-01 $1.54223 \mathrm{D}+0 \odot$

$\odot .99930002 .20868 \mathrm{D}-02$ 9.32514D-01 $1.54856 \mathrm{D}+00$

$0.99917803 .16701 \mathrm{D}-02$ 9.31426D-01 $1.57783 \mathrm{D}+00$

$\odot .99343004 .24375 \mathrm{D}-02$ 9.46053D-01 $1.59178 \mathrm{D}+00$

$0.99882006 .54989 \mathrm{D}-02$ 9.07049D-01 1.58294D+००

$1.00439007 .63665 \mathrm{D}-02$ 9.05001D-01 $1.57489 \mathrm{D}+00$

$1.00028001 .03764 \mathrm{D}-01$ 9.02012D-01 $1.59398 \mathrm{D}+00$

$1.00863001 .94540 \mathrm{D}-02 \quad 7.29655 \mathrm{D}-01$ 1.30433D+00

$\begin{array}{llll}1.0133800 & 3.54199 D-02 & 6.79294 D-01 & 1.26973 D+00\end{array}$

$1.01502007 .38332 \mathrm{D}-02$ 6.34551D-01 $1.23225 \mathrm{D}+00$

$\begin{array}{lllll}1.0167800 & 1.23994 D-01 & 6.38083 D-01 & 1.23857 D+0 \odot\end{array}$

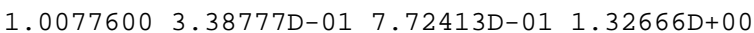

$0.99360506 .25216 \mathrm{D}-03$ 9.60877D-01 1.51053D+00

$1.00580001 .11059 \mathrm{D}-02$ 8.02747D-01 $1.36803 \mathrm{D}+00$

$1.00329001 .80910 \mathrm{D}-02$ 7.25977D-01 $1.29312 \mathrm{D}+00$

$1.00192001 .57911 \mathrm{D}-02 \quad 6.81190 \mathrm{D}-01$ 1.26673D+0๑

$\odot .99887001 .80840 \mathrm{D}-02 \quad 6.05322 \mathrm{D}-01 \quad 1.22233 \mathrm{D}+00$

$1.00597006 .36978 D-02$ 5.65441D-01 $1.16374 D+00$

$\odot .99398902 .49050 \mathrm{D}-02$ 5.70069D-01 $1.17707 \mathrm{D}+00$

$\odot .99991502 .16333 D-02 \quad 5.16334 D-01 \quad 1.14961 D+00$

1.0081500 4.01294D-02 5.47800D-01 $1.16428 D+00$

$1.00034001 .97271 D-01$ 1.94242D-01 9.24975D-01

1.0007800 2.88262D-01 1.05031D-02 7.15220D-01

$0.99355403 .45089 D-01$ 6.18867D-03 6.98348D-01

$0.99845101 .34778 D+00 \quad 8.05895 D-03 \quad 7.25057 D-01$

$0.99729903 .63087 D-01$ 5.22475D-03 6.86191D-01

$1.00063001 .24037 D+00 \quad 8.50638 D-03 \quad 7.32715 D-01$

$\odot .99449601 .25270 D+0 \odot \quad 9.00113 D-03 \quad 7.38190 D-01$

$0.99013901 .60894 D+00 \quad 2.55702 D-04 \quad 5.20954 D-01$

$0.99728901 .65212 \mathrm{D}+00 \quad 2.44033 \mathrm{D}-04$ 5.19066D-01

$0.9942520 \quad 6.23634 \mathrm{D}-03 \quad 9.35643 \mathrm{D}-01 \quad 1.49045 \mathrm{D}+00$

$\odot .99489403 .97495 \mathrm{D}-02$ 6. $64165 \mathrm{D}-01$ 1.20395D+००

1.0009700 4.31876D-02 5.85009D-01 1.19996D+00

$0.99891004 .81545 \mathrm{D}-02$ 5.57467D-01 1.17681D+0๑

1. 0033300 2.84721D-01 4.36411D-01 1.09113D+00

$1.00138003 .26193 \mathrm{D}-01$ 4.17643D-01 $1.07260 \mathrm{D}+00$

0.9933460 5.00934D-01 2.75229D-01 9.93519D-01

$0.99942905 .42136 \mathrm{D}-01$ 2.67003D-01 9.77602D-01

$\odot .98875301 .26740 \mathrm{D}-02 \quad 7.70222 \mathrm{D}-01 \quad 1.33928 \mathrm{D}+\odot \odot$

$0.99179802 .27282 D-02 \quad 6.25639 D-01 \quad 1.20816 D+00$

$1.00390003 .72855 \mathrm{D}-02$ 5.04949D-01 $1.12526 \mathrm{D}+00$

$0.99707803 .82474 D-01$ 1.29054D-01 8.67598D-01

$1.00223008 .28805 \mathrm{D}-01$ 1.70205D-03 6.11001D-01

$1.00617008 .82644 \mathrm{D}-03$ 8.83062D-01 $1.42961 \mathrm{D}+00$

$1.01282001 .08766 \mathrm{D}-02$ 8.19127D-01 $1.36904 \mathrm{D}+0 \odot$

$1.01746001 .30645 \mathrm{D}-02 \quad 7.71044 \mathrm{D}-01 \quad 1.31607 \mathrm{D}+00$

$\begin{array}{llll}1.0073500 & 8.17350 D+01 & 3.18382 D-01 & 9.82615 D-01\end{array}$

1. $00054008.03310 \mathrm{D}+01$ 9.69484D-02 8.45441D-01

$0.99934507 .83223 D+01 \quad 1.40322 D-02 \quad 7.24844 D-01$

$1.00320007 .74684 \mathrm{D}+01 \quad 8.51570 \mathrm{D}-03 \quad 7.05252 \mathrm{D}-01$

$\begin{array}{llll}1.0068100 & 7.73921 D+01 & 8.21040 D-03 & 7.02496 D-01\end{array}$

$1.00258007 .80263 \mathrm{D}+01$ 1.10903D-02 7.28138D-01

$\begin{array}{llll}1.00457007 .49133 D+01 & 3.32848 D-04 & 5.22499 D-01\end{array}$ $\odot .910$

$\odot .690$

5.280

4.610

8.090

8.450

7.080

7.050

1.590

1.300

3.090

2.880

5.250

5.360

5.200

2.780

2.920

5.280

6.230

12.920

3.880

3.380

4.280

5.390

5.220

6.450

5.060

4.340

4.280

7.200

5.050

11.330

9.770

8.470

9.310

9.500

9.440

10.730

2.700

4.310

5.360

4.530

4.550

5.830

4.720

6.910

4.060

4.860

6.360

10.020

11.690

4.120

5.810

6.390

33.970

33.470

37.410

38.420

47.470

32.080

43.380 


\begin{tabular}{|c|c|c|}
\hline HMFO०7-42 & U235 & Poly \\
\hline HMF○०7-43 & U235 & Poly \\
\hline HMF००8-1 & U235 & Bare \\
\hline HMF००9-1 & U235 & $\mathrm{Be}$ \\
\hline HMF००9-2 & U235 & $\mathrm{BeO}$ \\
\hline HMF010-1 & U235 & $\mathrm{B}-\mathrm{Be}$ \\
\hline HMF०10- 2 & U235 & $\mathrm{B}-\mathrm{BeO}$ \\
\hline HMF011-1 & U235 & Poly \\
\hline HMFO12-1 & U235 & $\mathrm{Al}$ \\
\hline HMF013-1 & U235 & steel \\
\hline HMF014-1 & U235 & $\mathrm{Al}$ \\
\hline Hmf015 & U235 & Bare \\
\hline Hmf016-1 & U235 & $\mathrm{Be}$ \\
\hline Hmf016- 2 & U235 & $\mathrm{BeO}$ \\
\hline Hmf017-1 & U235 & $\mathrm{Be}$ \\
\hline HMF018-D1 & U235 & Bare \\
\hline HMF018-S1 & U235 & Bare \\
\hline HMF019-D1 & U235 & Graphite \\
\hline HMF019-S1 & U235 & Graphite \\
\hline HMFO20-D1 & U235 & 1.45-Poly \\
\hline HMFO20-S1 & U235 & 1.45-Poly \\
\hline HMFO21-D1 & U235 & Steel \\
\hline HMFO21-S1 & U235 & Steel \\
\hline HMFO22-D1 & U235 & Duralumin \\
\hline $\mathrm{HMF} 022-\mathrm{S} 1$ & U235 & Duralumin \\
\hline HMFO24-1 & U235 & poly \\
\hline HMFO27 - 1 & U235 & lead \\
\hline HMFO28 & U235 & Flattop \\
\hline HMF०29-1 & U235 & Duralumin \\
\hline HMF○3० & U235 & D38 \\
\hline HMF031-1 & U235 & Poly \\
\hline HMF०32-1 & U235 & Unat \\
\hline HMF०32- 2 & U235 & Unat \\
\hline HMFO32-3 & U235 & Unat \\
\hline HMFO32- 4 & U235 & Unat \\
\hline Hmf०33-1 & U235 & Poly \\
\hline Hmf०33-2 & U235 & Poly \\
\hline Hmf034-1 & U235 & Poly-Ti \\
\hline $\mathrm{Hmf0} 34-2$ & U235 & Poly-Al \\
\hline $\mathrm{Hmf0} 34-3$ & U235 & Poly-steel \\
\hline Hmf036-1 & U235 & $\mathrm{CH} 2-\mathrm{D} 38$ \\
\hline Hmf036-2 & U235 & $\mathrm{CH} 2-\mathrm{D} 38$ \\
\hline Hmf037-1 & U235 & $\mathrm{CH} 2-\mathrm{D} 38$ \\
\hline $\mathrm{Hmf} 037-2$ & U235 & $\mathrm{CH} 2-\mathrm{D} 38$ \\
\hline $\mathrm{Hmf} 038-1$ & U235 & $\mathrm{Be}-\mathrm{D} 38$ \\
\hline Hmf०38-2 & U235 & $\mathrm{Be}-\mathrm{D} 38$ \\
\hline HMF०41-1 & U235 & $\mathrm{Be}$ \\
\hline HMFO41-2 & U235 & $\mathrm{Be}$ \\
\hline HMFO $41-3$ & U235 & Graphite \\
\hline HMF०41- 4 & U235 & Graphite \\
\hline HMF०41- 5 & U235 & Graphite \\
\hline HMF०41- 6 & U235 & Graphite \\
\hline HMF055-1 & U235 & ZPR3/23 \\
\hline HMF057-1 & U235 & lead \\
\hline HMF057- 2 & U235 & lead \\
\hline HMF057 - 3 & U235 & lead \\
\hline HMF057-4 & U235 & lead \\
\hline HMFO57-5 & U235 & lead \\
\hline HMF057- 6 & U235 & lead \\
\hline HMF058-1 & U235 & $\mathrm{Be}$ \\
\hline HMF058- 2 & U235 & $\mathrm{Be}$ \\
\hline HMF०58-3 & U235 & $\mathrm{Be}$ \\
\hline HMF058- 4 & U235 & $\mathrm{Be}$ \\
\hline HMF058-5 & U235 & $\mathrm{Be}$ \\
\hline HMF०60-1 & U235 & ZPR9/4-10 \\
\hline HMF061 & U235 & Graphite \\
\hline HMFO63-1 & U235 & LiD \\
\hline HMFO63-2 & U235 & LiD \\
\hline HMF064 - 1 & U235 & lead \\
\hline HMFO64 - 2 & U235 & lead \\
\hline HMF064-3 & U235 & lead \\
\hline
\end{tabular}

$0.99704707 .56283 D+01 \quad 2.80868 D-04 \quad 5.17306 D-01$ $0.99691007 .35115 \mathrm{D}+01$ 1.40345D-05 3.16054D-01 $\odot .99265801 .09080 \mathrm{D}-02$ 9.22593D-01 $1.47397 \mathrm{D}+00$ $0.99124801 .68059 \mathrm{D}-02 \quad 8.73407 \mathrm{D}-01 \quad 1.50885 \mathrm{D}+00$ $\odot .99797301 .60386 \mathrm{D}-02 \quad 8.50148 \mathrm{D}-01$ 1.45765D+00 $\odot .99611601 .82700 \mathrm{D}-02 \quad 8.82920 \mathrm{D}-01 \quad 1.47935 \mathrm{D}+00$ $\odot .99390401 .80533 \mathrm{D}-02 \quad 8.40401 \mathrm{D}-01 \quad 1.42520 \mathrm{D}+00$ $1.00320005 .18358 \mathrm{D}+01 \quad 4.96462 \mathrm{D}-01 \quad 1.10283 \mathrm{D}+00$ $1.00007001 .19639 \mathrm{D}-02$ 8.82988D-01 $1.45247 \mathrm{D}+00$ $\odot .99340201 .41418 \mathrm{D}-02 \quad 8.85016 \mathrm{D}-01$ 1.43536D+०० $0.99827501 .86965 \mathrm{D}-02 \quad 8.83980 \mathrm{D}-01 \quad 1.51022 \mathrm{D}+00$ $\odot .99615307 .15753 \mathrm{D}-03$ 9.65060D-01 $1.51602 \mathrm{D}+0 \odot$ $1.00106007 .37009 \mathrm{D}-01 \quad 8.75170 \mathrm{D}-01$ 1.51308D+00 $\begin{array}{llll}1.0070900 & 2.69599 \mathrm{D}-01 & 8.42394 \mathrm{D}-01 & 1.46355 \mathrm{D}+00\end{array}$ $0.99434701 .90606 \mathrm{D}-02$ 7.59648D-01 $1.44949 \mathrm{D}+00$ $1.00524006 .77743 \mathrm{D}-03 \quad 9.19355 \mathrm{D}-01$ 1.47320D+0๑ $0.99208706 .62328 \mathrm{D}-03 \quad 9.26107 \mathrm{D}-01 \mathrm{1} 1.47978 \mathrm{D}+00$ $1.01086001 .23151 D-02 \quad 8.67417 D-01 \quad 1.42863 D+00$ $1.00333001 .22922 \mathrm{D}-02$ 8.58561D-01 $1.41022 \mathrm{D}+00$ $\odot .99974301 .31934 \mathrm{D}-01$ 8.01151D-01 $1.37041 \mathrm{D}+00$ 1. $00128001.22463 \mathrm{D}-01$ 7.96219D-01 $1.37031 \mathrm{D}+00$ $0.99798802 .25875 \mathrm{D}-02$ 8.32496D-01 $1.38504 \mathrm{D}+00$ $0.99462602 .27892 \mathrm{D}-02$ 8.30836D-01 $1.38728 \mathrm{D}+00$ $1.00246001 .09647 \mathrm{D}-02 \quad 8.65618 \mathrm{D}-01 \quad 1.43606 \mathrm{D}+00$ $1.00375001 .09851 D-02$ 8.69976D-01 $1.44085 D+0 \odot$ $\begin{array}{llll}1.0002100 & 5.14092 D+01 & 6.31509 D-01 & 1.20849 D+00\end{array}$ $1.00683001 .01518 D-02 \quad 8.91335 D-011.45218 D+00$ $1.00429006 .63269 \mathrm{D}-02$ 9.18332D-01 $1.59074 \mathrm{D}+00$ $1.00592001 .49636 \mathrm{D}-02$ 8.98457D-01 $1.51658 \mathrm{D}+00$ $1.00487002 .05447 D-01 \quad 4.79038 D-01 \quad 1.40656 D+00$ $\begin{array}{llll}1.0075600 & 8.22255 D+01 & 2.40976 D-01 & 9.33050 D-01\end{array}$ $1.00343003 .10353 \mathrm{D}-02$ 9.32997D-01 $1.57467 \mathrm{D}+00$ $1.00492002 .71014 \mathrm{D}-02$ 9.18925D-01 $1.55948 \mathrm{D}+00$ $1.00065001 .32869 \mathrm{D}-02$ 9.22328D-01 $1.52120 \mathrm{D}+0 \odot$ 1.0055200 8.24981D-03 9.43564D-01 1.51065D+00 $1.00476003 .80786 \mathrm{D}-01$ 1.06510D-01 8.25606D-01 $0.97583602 .00568 D+00 \quad 9.61611 D-03 \quad 7.19616 D-01$ $\odot .99204301 .43370 D+00 \quad 2.30129 D-01$ 9.15511D-01 $0.99714901 .25551 D+00 \quad 2.23869 D-01$ 9.15240D-01

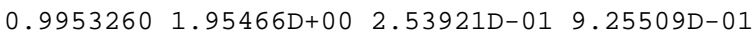
$1.00283001 .95486 \mathrm{D}+00$ 4.42945D-02 8.39253D-01 $\odot .99843301 .62350 \mathrm{D}+00 \quad 3.74682 \mathrm{D}-01 \quad 1.07399 \mathrm{D}+00$ $\odot .99768906 .06753 \mathrm{D}+01$ 9.81247D-02 8.96765D-01 $0.99803203 .84388 \mathrm{D}+01 \quad 2.70072 \mathrm{D}-01 \quad 1.00354 \mathrm{D}+00$ $\begin{array}{llll}1.0100200 & 2.03722 \mathrm{D}-01 & 4.93179 \mathrm{D}-01 & 1.40034 \mathrm{D}+0 \odot\end{array}$ $\begin{array}{llll}1.0072400 & 2.33186 \mathrm{D}-01 & 3.32367 \mathrm{D}-01 & 1.30704 \mathrm{D}+00\end{array}$ 1.0049600 3.94349D-02 8.21549D-01 1.49441D+00 $0.99428801 .17061 \mathrm{D}+01 \quad 7.28678 \mathrm{D}-01 \quad 1.53395 \mathrm{D}+00$ $1.00209001 .51341 \mathrm{D}-02 \quad 8.66603 \mathrm{D}-01$ 1.40333D+०० $1.01413006 .44422 \mathrm{D}-02 \quad 7.79220 \mathrm{D}-01$ 1.33301D+00 $1.00632008 .56427 \mathrm{D}-01 \quad 7.37033 \mathrm{D}-01$ 1.29137D+00 $1.00487006 .45749 D+00 \quad 6.97247 D-01 \quad 1.25151 D+00$ $0.99673702 .15335 \mathrm{D}-01 \quad 3.97225 \mathrm{D}-01$ 1.05282D+00 $0.99177804 .12411 \mathrm{D}-02$ 8.59219D-01 $1.42260 \mathrm{D}+0 \odot$ $\odot .99216901 .89624 \mathrm{D}-02 \quad 8.74632 \mathrm{D}-01$ 1.42922D+0๑ $1.01819002 .78480 \mathrm{D}-02 \quad 8.46890 \mathrm{D}-01$ 1.39109D+00 $0.98920201 .90113 \mathrm{D}-02$ 8.97325D-01 $1.45493 \mathrm{D}+00$ $1.02526003 .34403 \mathrm{D}-02 \quad 8.39551 \mathrm{D}-01 \quad 1.39183 \mathrm{D}+00$ $0.99113003 .10124 \mathrm{D}-02$ 8.54970D-01 $1.41502 \mathrm{D}+00$ $\odot .99933901 .31590 \mathrm{D}+02 \quad 6.35584 \mathrm{D}-01 \quad 1.52597 \mathrm{D}+00$ $1.00577003 .15598 \mathrm{D}+00 \quad 7.62693 \mathrm{D}-01$ 1.52676D+00 $0.99803808 .67433 \mathrm{D}-02 \quad 8.25217 \mathrm{D}-01$ 1.51322D+00 $\odot .99816701 .50512 \mathrm{D}-02$ 8.65241D-01 $1.50923 \mathrm{D}+00$ $\odot .99715101 .03686 \mathrm{D}-02 \quad 8.99627 \mathrm{D}-01$ 1.51572D+00 $\begin{array}{llll}1.0190900 & 2.45611 D-01 & 2.22987 D-01 & 6.06550 D-01\end{array}$ 1.0123900 2.67721D+00 4.36929D-01 9.72699D-01 $1.01401001 .08146 \mathrm{D}-02$ 8.47098D-01 $1.42051 \mathrm{D}+00$ $1.00285001 .28355 \mathrm{D}-02 \quad 8.17429 \mathrm{D}-01$ 1.38665D+00 $0.99042803 .65417 \mathrm{D}-02$ 8.57962D-01 $1.40738 \mathrm{D}+00$

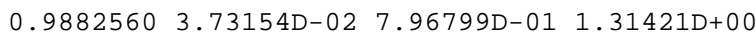
$\odot .98839703 .79943 D-02$ 8.03994D-01 $1.30964 D+\odot \odot$
40.120 35.280 4.200

4.690

5.420

6.580

6.050

19.030

3.880

6.880

8.230

2.670

3.090

3.050

1.640

2.310

2.480

2.800

3.110

3. 270

3.090

9.980

7.270

2.810

2.730

22.770

4.500

6.450

5.980

18.730

36.840

3.530

1.950

1.500

1.090

7.970

8.230

5.340

7.020

8.750

8.250

5.060

25.970

23.390

12.310

16.360

1.560

3.410

1.190

1.220

2.030

2.730

65.270

4.030

2.250

5.280

3.160

4.720

5.530

10.340

1.800

1.830

1.170

1.220

51.590

86.770

1.030

1.330

7.200

6.360

7.200 


\begin{tabular}{|c|c|c|c|c|c|c|c|}
\hline HMF065 & U235 & Bare & 1.0030000 & 7. $04647 \mathrm{D}-03$ & $9.68307 \mathrm{D}-01$ & $1.52033 \mathrm{D}+00$ & 2.330 \\
\hline HMF066 - 1 & U235 & $\mathrm{Be}$ & 0.9986410 & $2.17961 D+\odot \odot$ & $7.00151 \mathrm{D}-01$ & $1.50147 \mathrm{D}+00$ & 2.950 \\
\hline HMF066 - 2 & U235 & $\mathrm{Be}$ & 0.9981100 & $8.53898 D-02$ & $7.75258 D-01$ & $1.49418 \mathrm{D}+0 \odot$ & 2.220 \\
\hline HMF๑66-3 & U235 & $\mathrm{Be}$ & $\odot .9952260$ & $2.22217 \mathrm{D}-02$ & $8.14597 \mathrm{D}-01$ & $1.49317 \mathrm{D}+\odot \odot$ & 1.120 \\
\hline HMF066 - 4 & U235 & $\mathrm{Be}$ & 1.0048100 & $2.29627 D+01$ & $5.37974 \mathrm{D}-01$ & $1.46102 \mathrm{D}+00$ & 3.920 \\
\hline HMF०666- 5 & U235 & $\mathrm{Be}$ & 1.0025500 & $1.22474 \mathrm{D}+\odot \odot$ & $6.68429 \mathrm{D}-01$ & $1.47743 \mathrm{D}+\odot \odot$ & 2.480 \\
\hline HMF066- 6 & U235 & $\mathrm{Be}$ & $\odot .990040 \odot$ & $1.25079 D-\odot 1$ & $7.25743 \mathrm{D}-01$ & $1.48697 \mathrm{D}+\odot \odot$ & 1.690 \\
\hline HMF๑66-7 & U235 & $\mathrm{Be}$ & 1. $\odot \odot 11900$ & $1 . \odot 2 \odot 67 D+\odot \odot$ & $6.42749 \mathrm{D}-01$ & $1.46360 \mathrm{D}+\odot \odot$ & 2.480 \\
\hline HMF๑66-8 & U235 & $\mathrm{Be}$ & 1.0033300 & $7.82435 \mathrm{D}+\odot \odot$ & $5.31911 \mathrm{D}-01$ & $1.435 \odot 2 \mathrm{D}+\odot \odot$ & 3.670 \\
\hline HMF066 - 9 & U235 & $\mathrm{Be}$ & 1.0008200 & $1.68318 \mathrm{D}-01$ & $6.13194 \mathrm{D}-01$ & $1.41565 \mathrm{D}+0 \odot$ & 2.170 \\
\hline HMF067-1 & U235 & ZPR9-5 & 1.0120200 & $3.15703 \mathrm{D}-01$ & $1.21961 \mathrm{D}-01$ & $5.10357 \mathrm{D}-01$ & 61.340 \\
\hline HMF०67-2 & U235 & ZPR9- 6 & 1.0060800 & $2.97908 \mathrm{D}-01$ & $2.05825 \mathrm{D}-\odot 1$ & $5.84251 D-01$ & 63.610 \\
\hline HMFO7०-1 & U235 & ZPR9- 7 & 1.0189900 & $1.65346 \mathrm{D}+01$ & $1.29431 \mathrm{D}-01$ & $5.09669 D-01$ & 86.000 \\
\hline HMF०7०-2 & U235 & ZPR9-8 & 1.0139700 & $7.82103 \mathrm{D}+\odot \odot$ & $1.34488 \mathrm{D}-01$ & $5.86866 \mathrm{D}-01$ & 90.050 \\
\hline HMFO7๑- 3 & U235 & ZPR9-9 & 1.0084600 & $4.19265 D+01$ & $1.15168 \mathrm{D}-01$ & 4. $92898 D-01$ & 109.340 \\
\hline Hmf०73-1 & U235 & $\mathrm{Cu}$ & 1.0052300 & $2.56386 \mathrm{D}-01$ & $4.64840 \mathrm{D}-01$ & $9.9 \odot \odot 17 \mathrm{D}-\odot 1$ & 43.380 \\
\hline Hmf०78-1 & U235 & water & 0.9946510 & $2.18079 \mathrm{D}+01$ & $6.84541 \mathrm{D}-01$ & $1.25610 \mathrm{D}+\odot \odot$ & 9.910 \\
\hline Hmf078-2 & U235 & Poly & 0.9876160 & $6.72179 D-01$ & $7.98046 \mathrm{D}-01$ & $1.37150 \mathrm{D}+0 \odot$ & 2.120 \\
\hline Hmf०78-3 & U235 & Poly & 0.9943280 & $5.99828 D+\odot \odot$ & $7.15479 \mathrm{D}-01$ & $1.28454 \mathrm{D}+\odot \odot$ & 4.750 \\
\hline Hmf078-4 & U235 & Poly & 1.0010600 & $1.29207 \mathrm{D}+01$ & $3.44936 \mathrm{D}-01$ & $1.00653 \mathrm{D}+0 \odot$ & 7.750 \\
\hline Hmf078-5 & U235 & Poly & 0.9971040 & $1.33760 \mathrm{D}+01$ & $6.60379 D-01$ & $1.24966 \mathrm{D}+\odot \odot$ & 6.660 \\
\hline Hmf०78-6 & U235 & Poly & $\odot .9981170$ & $1.84781 \mathrm{D}+01$ & $6.52187 \mathrm{D}-01$ & $1.23524 \mathrm{D}+\odot \odot$ & 10.580 \\
\hline Hmf078- 7 & U235 & Poly & 1.0077700 & $2.27141 \mathrm{D}+01$ & $6.63659 D-01$ & $1.25219 \mathrm{D}+\odot \odot$ & 11.030 \\
\hline Hmf०78-8 & U235 & Poly & 0.9997890 & $2.40139 D+01$ & $6.71947 \mathrm{D}-01$ & $1.24779 D+\odot \odot$ & 14.420 \\
\hline Hmf०78-9 & U235 & Poly & 1.0005100 & $2.39314 \mathrm{D}+01$ & $6.47741 \mathrm{D}-01$ & $1.2224 \odot \mathrm{D}+\odot \odot$ & 13.300 \\
\hline Hmf078-10 & U235 & Lucite & 0.9987630 & $2.28311 \mathrm{D}+01$ & $6.55136 \mathrm{D}-01$ & $1.23451 \mathrm{D}+00$ & 9.910 \\
\hline Hmf078-11 & U235 & Paraffin & 1.0030300 & $2.29025 \mathrm{D}+01$ & $6.62889 \mathrm{D}-01$ & $1.24050 D+\odot \odot$ & 12.670 \\
\hline Hmf078-12 & U235 & Graphite & 0.9982000 & $8.04287 \mathrm{D}-03$ & $9.34476 \mathrm{D}-01$ & $1.47807 \mathrm{D}+\odot \odot$ & 1.480 \\
\hline Hmf078-13 & U235 & Graphite & 1.0055100 & 1. $05449 D-02$ & $9.07595 \mathrm{D}-01$ & $1.45199 \mathrm{D}+\odot \odot$ & 1.670 \\
\hline Hmf078-14 & U235 & Graphite & 0.9955520 & $5.81496 \mathrm{D}-02$ & $8.48613 \mathrm{D}-01$ & $1.39985 \mathrm{D}+0 \odot$ & 1.750 \\
\hline Hmf078-15 & U235 & Graphite & 1.0074700 & $1.59410 \mathrm{D}-01$ & $6.88954 \mathrm{D}-01$ & $1.23847 \mathrm{D}+0 \odot$ & 2.880 \\
\hline Hmf078-16 & U235 & Graphite & $\odot .9914680$ & 1. $05565 D-01$ & $8.47978 \mathrm{D}-01$ & $1.38395 D+\odot \odot$ & 1.590 \\
\hline Hmf078-17 & U235 & graphite & 1.0019300 & $2.82483 D-01$ & $6.60907 \mathrm{D}-01$ & $1.21252 \mathrm{D}+\odot \odot$ & 3.300 \\
\hline Hmf078-18 & U235 & Graphite & 0.9922660 & $1.35742 \mathrm{D}-01$ & $8.67208 D-01$ & $1.40781 \mathrm{D}+\odot \odot$ & 1.670 \\
\hline Hmf078-19 & U235 & Graphite & 0.9958440 & $4.66875 \mathrm{D}-01$ & $8.43887 \mathrm{D}-01$ & $1.38714 \mathrm{D}+\odot \odot$ & 2.190 \\
\hline Hmf078-20 & U235 & Graphite & 0.9980550 & $7.46569 D-01$ & $8.46771 \mathrm{D}-01$ & $1.39048 \mathrm{D}+0 \odot$ & 2.620 \\
\hline Hmf078-21 & U235 & Graphite & 1.0006000 & $6.45679 D-03$ & $9.68865 \mathrm{D}-01$ & $1.51732 D+\odot \odot$ & 1.160 \\
\hline Hmf078- 22 & U235 & Poly & 1.0014800 & $1.90113 \mathrm{D}+01$ & $8.41368 \mathrm{D}-01$ & $1.40 \odot \odot 5 \mathrm{D}+\odot \odot$ & 6.480 \\
\hline HMFO79-1 & U235 & $\mathrm{Ti}$ & 1.0050900 & $8.56479 \mathrm{D}-03$ & $9.45186 \mathrm{D}-01$ & $1.49438 \mathrm{D}+00$ & 4.520 \\
\hline HMF०79-2 & U235 & Ti & 1.0029200 & $9.45162 \mathrm{D}-03$ & $9.4615 \odot \mathrm{D}-\odot 1$ & $1.49307 \mathrm{D}+\odot \odot$ & 4.640 \\
\hline HMFO79-3 & U235 & $\mathrm{Ti}$ & 1.0009000 & 1. $06772 \mathrm{D}-02$ & $9.32927 D-01$ & $1.48314 \mathrm{D}+00$ & 5.110 \\
\hline HMFO79- 4 & U235 & $\mathrm{Ti}$ & 1.0053600 & 1. 39248D- 02 & $9.05802 \mathrm{D}-01$ & $1.45174 \mathrm{D}+00$ & 5.270 \\
\hline HMF079-5 & U235 & $\mathrm{Ti}$ & 1.0037000 & $1.58609 \mathrm{D}-\odot 2$ & $9.27442 \mathrm{D}-01$ & $1.47104 \mathrm{D}+\odot \odot$ & 4.810 \\
\hline HMM००1 & U235 & Poly & 1.0072800 & $5.50150 D+01$ & $3.95761 \mathrm{D}-02$ & $7.73642 \mathrm{D}-01$ & 33.690 \\
\hline HMM००2 & U235 & Poly & 1.0131400 & $6.89679 D+01$ & $1.23815 \mathrm{D}-\odot 2$ & $7.63585 D-01$ & 28.560 \\
\hline НMM००З & U235 & Poly & 1.0040600 & $7.87708 \mathrm{D}+01$ & $1.43584 \mathrm{D}-02$ & $7.76677 D-01$ & 42.280 \\
\hline НMM००4 & U235 & Poly & 1.0054100 & $1.67924 D+01$ & $1.10031 \mathrm{D}-03$ & $9.37421 D-01$ & 14.890 \\
\hline IMF๑०2 & U235 & Cylindrical-D & 1.0046000 & $6.19718 D-02$ & $4.82997 D-01$ & $1.29690 \mathrm{D}+\odot \odot$ & 5.270 \\
\hline IMF००3 & U235 & Spherical & 1.0050300 & 1. 78961D-०2 & $6.36276 \mathrm{D}-01$ & $1.33449 \mathrm{D}+00$ & 8.970 \\
\hline 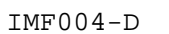 & U235 & Graphite & 1.0042700 & $2.35308 \mathrm{D}-\odot 2$ & $6.07220 \mathrm{D}-\odot 1$ & $1.29595 \mathrm{D}+\odot \odot$ & 4.950 \\
\hline IMF००4-S & U235 & Graphite & 1.0052800 & $2.35583 D-\odot 2$ & $5.98196 \mathrm{D}-\odot 1$ & $1.29540 \mathrm{D}+\odot \odot$ & 6.060 \\
\hline IMF०05 & U235 & Spherical-D & 0.9978030 & $3.38093 \mathrm{D}-02$ & $5.98376 \mathrm{D}-01$ & $1.27716 \mathrm{D}+\odot \odot$ & 15.810 \\
\hline IMF๑๑6 & U235 & Spherical-D & $\odot .9955450$ & 4. $\odot 95 \odot 9 D-\odot 2$ & $5.85375 \mathrm{D}-01$ & $1.26180 \mathrm{D}+\odot \odot$ & 15.910 \\
\hline IMF००8 & U235 & Spherical-D & 1.0010000 & $2.32711 \mathrm{D}-02$ & $6.32529 D-01$ & $1.35086 \mathrm{D}+00$ & 9.340 \\
\hline IMF००9 & U235 & Spherical-D & 1.0145600 & 2. $02584 \mathrm{D}+01$ & $3.06045 \mathrm{D}-01$ & $9.92893 \mathrm{D}-01$ & 15.590 \\
\hline IMF010-1 & U235 & U9 & 0.9980800 & $1.63371 \mathrm{D}-01$ & $3.35834 \mathrm{D}-01$ & $1.17779 \mathrm{D}+\odot \odot$ & 73.280 \\
\hline IMF012-1 & U235 & ZPR-3/41 & 0.9999420 & $2.25655 \mathrm{D}-01$ & $3.17191 \mathrm{D}-01$ & $1.04846 D+\odot \odot$ & 61.250 \\
\hline IMF013-1 & U235 & ZPR-9/1 & 0.9950670 & $2.23 \odot 43 D-\odot 1$ & $3.51605 \mathrm{D}-01$ & $1.14137 \mathrm{D}+\odot \odot$ & 56.230 \\
\hline IMF014-1 & U235 & ZPR $-9 / 2$ & 0.9986400 & $2.20880 \mathrm{D}-01$ & $2.84848 D-01$ & $9.61201 \mathrm{D}-01$ & 60.470 \\
\hline IMF014-2 & U235 & ZPR $-9 / 3$ & 1.0075300 & 2. $\odot 8236 D-\odot 1$ & $2.41076 \mathrm{D}-01$ & $8.43905 \mathrm{D}-01$ & 64.440 \\
\hline SFIR๑०3 & U235 & Godiva & 1.0040800 & $6.26347 \mathrm{D}-03$ & $9.43592 \mathrm{D}-01$ & $1.5 \odot 949 \mathrm{D}+\odot \odot$ & 0.950 \\
\hline SFIR005 & U235 & Big10 & 0.9939040 & 1. $08082 D-01$ & $3.96133 \mathrm{D}-01$ & $1.28051 \mathrm{D}+00$ & 6.700 \\
\hline SFIR๑०6 & U235 & Flattop-25 & 1.0023900 & $6.67486 \mathrm{D}-02$ & $9.30487 \mathrm{D}-01$ & $1.60373 \mathrm{D}+\odot \odot$ & 4.920 \\
\hline HMFO०4-S & U235 & Water & 1.0010400 & 1. $06169 D+02$ & $5.32998 D-01$ & $1.11815 \mathrm{D}+0 \odot$ & 24.440 \\
\hline HMF○९4-D & U235 & water & 1.0014600 & 1. $05085 \mathrm{D}+02$ & $5.25945 \mathrm{D}-01$ & $1.11850 \mathrm{D}+0 \odot$ & 46.050 \\
\hline ICF००1 & U235 & Zpr6-6a & 0.9916890 & $5.83508 \mathrm{D}-01$ & $8.24672 \mathrm{D}-\odot 2$ & $6.40952 \mathrm{D}-01$ & 107.520 \\
\hline & & & & & & & \\
\hline \multicolumn{8}{|c|}{ 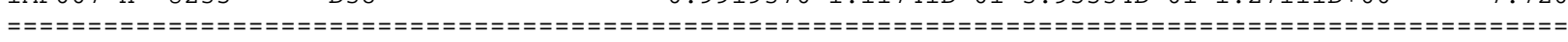 } \\
\hline & & Average & 1.0010058 & $+/-\quad 0.0052$ & 2317 & Total & 2629.610 \\
\hline & & & 0.9758360 & $-0.0<3$ & -698 (Trom & (erage) & \\
\hline & & Highest & 1.0252600 & $0.0<4<-2$ & 2542 (from Av & verage) & \\
\hline
\end{tabular}




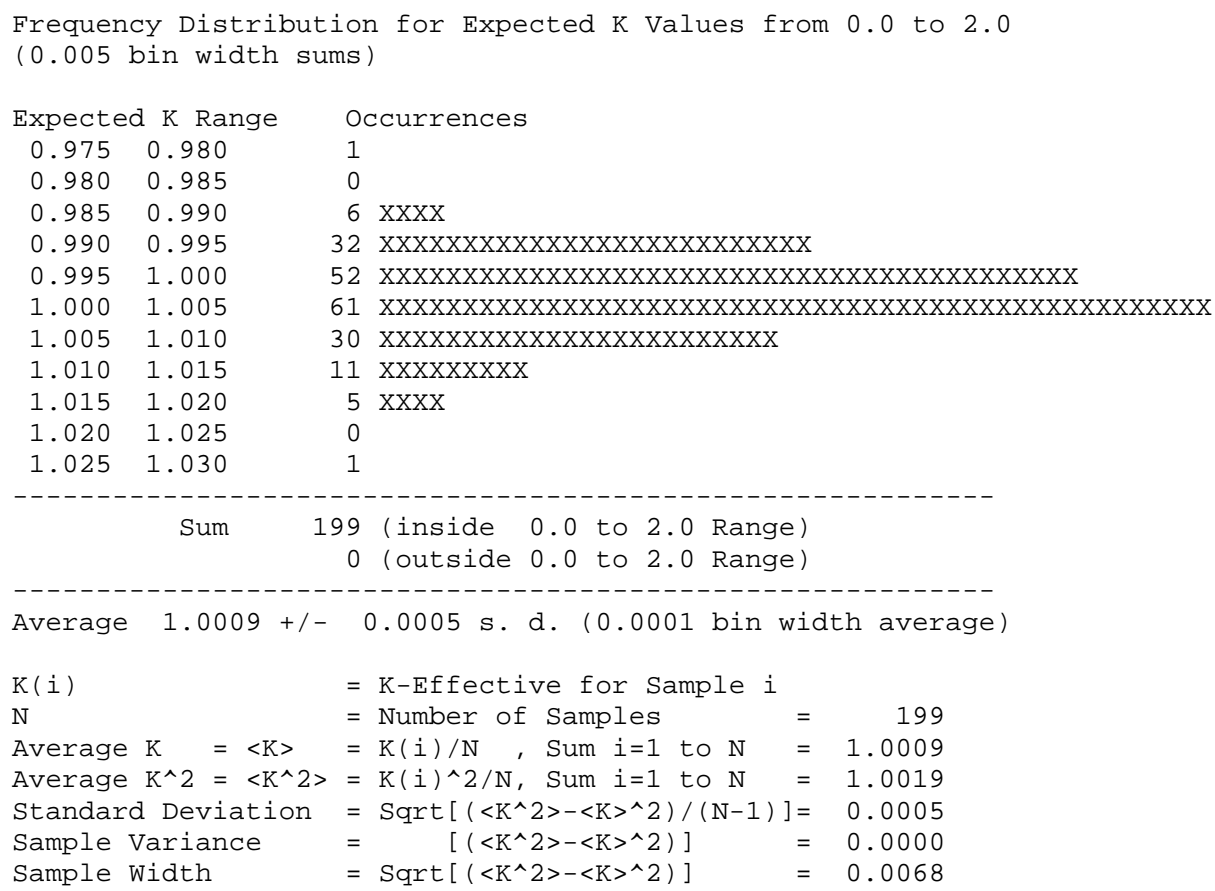

As $\mathrm{N}$ Approaches Infinity Standard Deviation Approaches Zero.

As $\mathrm{N}$ Approaches Infinity Sample width Approaches a Constant.

Confidence Limits

(Occurrences out to +/- 10 times Sample Width) $\begin{array}{lll}\text { Sample width } & \text { 0ccurrences } & \text { Per-Cent } \\ \text { Range } & \text { Occurred } & \text { Normal }\end{array}$

\begin{tabular}{rrrrr}
-4 & -3 & 1 & 0.503 & 0.132 \\
-2 & -1 & 28 & 14.070 & 13.591 \\
-1 & 0 & 75 & 37.688 & 34.134 \\
0 & 1 & 74 & 37.186 & 34.134 \\
1 & 2 & 14 & 7.035 & 13.591 \\
2 & 3 & 6 & 3.015 & 2.140 \\
3 & 4 & 1 & 0.503 & 0.132 \\
\hline & \multicolumn{2}{c}{ Sum } & 199 &
\end{tabular}




\section{U235 Slow (372 Assemblies)}

Criticality Calculation Editor (CRITEDIT 2002-1)

\begin{tabular}{|c|c|c|c|c|c|c|c|c|}
\hline $\begin{array}{l}\text { Crit. } \\
\text { ID. }\end{array}$ & Fuel & $\begin{array}{l}\text { Reflector } \\
\text { or Name }\end{array}$ & & Expected K & $\begin{array}{l}\text { Removal } \\
\text { Lifetime } \\
\text { (Microsec.) }\end{array}$ & $\begin{array}{l}\text { Median } \\
\text { Energy } \\
(\text { MeV) }\end{array}$ & $\begin{array}{l}\text { Average } \\
\text { Energy } \\
(\mathrm{MeV})\end{array}$ & Seconds \\
\hline \multicolumn{9}{|c|}{ 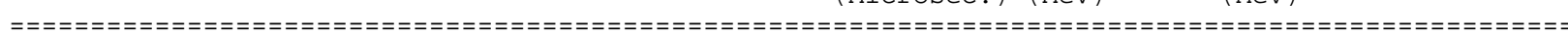 } \\
\hline HMT००1-1 & U235 & Poly & & 1.0080700 & $6.52976 \mathrm{D}+01$ & $4.93650 \mathrm{D}-08$ & $2.19777 \mathrm{D}-02$ & 31.800 \\
\hline HMT००८-D & U235 & Poly & & 1.0125700 & $6.34489 D+01$ & $4.96781 D-08$ & $2.20237 \mathrm{D}-02$ & 51.580 \\
\hline HMT००८-S & U235 & Poly & & 1.0138900 & $6.31692 \mathrm{D}+01$ & $5.03483 D-08$ & $2.08222 D-02$ & 61.690 \\
\hline HST००1-1 & U235 & Bare & & $\odot .9924440$ & $9.58781 \mathrm{D}+\odot \odot$ & $4.76672 \mathrm{D}-08$ & $1.52600 \mathrm{D}-02$ & 25.170 \\
\hline 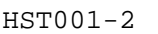 & U235 & Bare & & $\odot .9938250$ & $4.06571 D+\odot \odot$ & $8.53190 \mathrm{D}-\odot 8$ & $3.99813 \mathrm{D}-\odot 2$ & 20.940 \\
\hline HST००1 - 3 & U235 & Bare & & 1.0079000 & $9.87678 \mathrm{D}+\odot \odot$ & $4.68030 \mathrm{D}-08$ & $1.70385 \mathrm{D}-02$ & 21.200 \\
\hline HST००1 - 4 & U235 & Bare & & 1.0044200 & $3.94729 \mathrm{D}+\odot \odot$ & $8.91416 \mathrm{D}-08$ & $4.14049 \mathrm{D}-02$ & 16.890 \\
\hline HSTO०1- 5 & U235 & Bare & & 0.9987230 & $2.52065 \mathrm{D}+01$ & $3.64407 \mathrm{D}-08$ & $6.66791 \mathrm{D}-03$ & 21.410 \\
\hline HST००1- 6 & U235 & Bare & & 1.0058400 & $2.34902 \mathrm{D}+01$ & $3.68597 D-08$ & $6.83186 \mathrm{D}-03$ & 25.200 \\
\hline 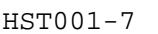 & U235 & Bare & & $\odot .9918910$ & $1.03942 D+01$ & $4.6 \odot 941 D-\odot 8$ & $1.66338 \mathrm{D}-\odot 2$ & 16.940 \\
\hline HST००1 - 8 & U235 & Bare & & ๑.9899480 & $9.82914 \mathrm{D}+\odot \odot$ & $4.76136 \mathrm{D}-08$ & $1.74800 \mathrm{D}-02$ & 19.230 \\
\hline HST००1- 9 & U235 & Bare & & $\odot .9923360$ & $4.07 \odot 24 D+\odot \odot$ & $8.97716 \mathrm{D}-08$ & $3.99923 \mathrm{D}-02$ & 15.890 \\
\hline HSTO०1 - 10 & U235 & Bare & & ๑.9875710 & $2.19682 D+01$ & $3.68633 \mathrm{D}-\odot 8$ & $8.94846 \mathrm{D}-03$ & 18.770 \\
\hline HSTO०2 - 1 & U235 & Steel & case & 1.0034200 & $2.89716 \mathrm{D}+02$ & $4.68288 D-08$ & $1.64808 \mathrm{D}-02$ & 141.550 \\
\hline 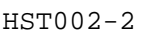 & U235 & Steel & case & 1. 0069100 & $2.52895 \mathrm{D}+02$ & $4.68458 \mathrm{D}-\odot 8$ & $1.57337 \mathrm{D}-\odot 2$ & 136.550 \\
\hline HSTO०2 - 3 & U235 & Steel & case & $\odot .9961150$ & $2.77507 \mathrm{D}+\odot 2$ & $8.13354 \mathrm{D}-08$ & $3.68863 \mathrm{D}-02$ & 140.730 \\
\hline HSTO०2 - 4 & U235 & Steel & case & 1.0067100 & $2.43061 \mathrm{D}+\odot 2$ & 8. $\odot \odot \odot 99 D-\odot 8$ & $3.47844 \mathrm{D}-02$ & 145.910 \\
\hline HSTO०2 - 5 & U235 & $\mathrm{Al}$ & case & 1.0057700 & $2.97783 \mathrm{D}+\odot 2$ & $4.70375 \mathrm{D}-\odot 8$ & $1.61727 \mathrm{D}-02$ & 141.780 \\
\hline HST००2 - 6 & U235 & Al & case & 1.0147000 & $2.64618 \mathrm{D}+02$ & $4.66839 D-\odot 8$ & $1.54925 \mathrm{D}-02$ & 108.920 \\
\hline 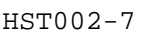 & U235 & $\mathrm{Al}$ & case & $\odot .9982340$ & $2.84627 D+02$ & 8. $01044 \mathrm{D}-\odot 8$ & $3.79413 \mathrm{D}-\odot 2$ & 127.140 \\
\hline HSTO०2 - 8 & U235 & Al & case & 1.0018800 & $2.53573 \mathrm{D}+\odot 2$ & $7.80164 \mathrm{D}-08$ & $3.60701 \mathrm{D}-02$ & 105.420 \\
\hline HST००2 - 9 & U235 & Al & case & 1. $0 \odot \odot \odot 10 \odot$ & $2.93561 \mathrm{D}+\odot 2$ & $3.68684 \mathrm{D}-08$ & $8.03246 D-\odot 3$ & 93.660 \\
\hline HSTO०2 - 10 & U235 & Al & case & 1.0036500 & $2.56629 D+\odot 2$ & $3.64709 \mathrm{D}-\odot 8$ & $8.0 \odot 6 \odot 4 D-\odot 3$ & 85.270 \\
\hline HSTOO2 - 11 & U235 & Al & case & 1.0074800 & 3. $01850 D+02$ & $4.63528 D-08$ & $1.53723 D-02$ & 82.860 \\
\hline HST००2 - 12 & U235 & $\mathrm{Al}$ & case & 1. $010280 \odot$ & $2.54929 \mathrm{D}+02$ & $4.65151 \mathrm{D}-\odot 8$ & $1.72168 \mathrm{D}-\odot 2$ & 108.890 \\
\hline HSTO०2 - 13 & U235 & Al & case & 1.0011600 & $2.85052 \mathrm{D}+\odot 2$ & $8.02772 D-\odot 8$ & $3.7 \odot 463 \mathrm{D}-\odot 2$ & 148.190 \\
\hline HSTO०2 - 14 & U235 & $\mathrm{Al}$ & case & 1.0023400 & $2.50 \odot 25 \mathrm{D}+\odot 2$ & $7.66576 \mathrm{D}-\odot 8$ & $3.52129 \mathrm{D}-02$ & 142.550 \\
\hline HST๑०3-1 & U235 & Poly & & 1.0021700 & $2.5 \odot 433 D+\odot 2$ & $3.66219 \mathrm{D}-\odot 8$ & $6.68907 \mathrm{D}-\odot 3$ & 66.670 \\
\hline Hst००3-2 & U235 & Poly & & 1.0024900 & $2.89242 \mathrm{D}+02$ & $3.64857 D-08$ & $7.23989 D-03$ & 59.520 \\
\hline Hst@o3-3 & U235 & Poly & & $\odot .9998930$ & $3.08122 D+\odot 2$ & $4.71318 D-08$ & 1.56687D-02 & 50.080 \\
\hline Hst०००-4 & U235 & Poly & & 1.0017100 & $2.51867 D+\odot 2$ & $4.65729 D-\odot 8$ & 1.51483D-๑2 & 75.910 \\
\hline Hst@o3-5 & U235 & Poly & & $\odot .9962780$ & $2.97046 \mathrm{D}+\odot 2$ & $8.26255 D-\odot 8$ & $3.82359 \mathrm{D}-02$ & 71.940 \\
\hline Hst@०3-6 & U235 & Poly & & 1.0004200 & $2.41220 \mathrm{D}+02$ & $7.93693 \mathrm{D}-\odot 8$ & $3.70222 \mathrm{D}-02$ & 65.800 \\
\hline Hst००3-7 & U235 & Poly & & 1.0029100 & $2.73291 \mathrm{D}+02$ & $3.64370 \mathrm{D}-\odot 8$ & $6.80822 D-03$ & 45.500 \\
\hline Hst@०3-8 & U235 & Poly & & 1.0015800 & $3.23079 \mathrm{D}+02$ & $4.69826 D-\odot 8$ & $1.70 \odot 7 \odot \mathrm{D}-\odot 2$ & 68.450 \\
\hline Hst๑०3-9 & U235 & Poly & & 1.0034300 & $2.71496 \mathrm{D}+\odot 2$ & $4.61345 \mathrm{D}-\odot 8$ & $1.53141 \mathrm{D}-02$ & 69.830 \\
\hline Hst०03-10 & U235 & Poly & & $\odot .9972190$ & $3.07251 D+02$ & $8.18035 \mathrm{D}-08$ & $3.60868 \mathrm{D}-02$ & 73.090 \\
\hline Hst003-11 & U235 & Poly & & 1.0011700 & $2.57248 \mathrm{D}+02$ & $7.74920 \mathrm{D}-\odot 8$ & $3.41697 \mathrm{D}-02$ & 78.690 \\
\hline Hst003-12 & U235 & Poly & & 1320 & $90 D+02$ & $3.665 \odot 2 \mathrm{D}-\odot 8$ & $7.74 \odot 45 \mathrm{D}-\odot 3$ & 61.810 \\
\hline Hst@०3-13 & U235 & Poly & & 1.0082300 & $2.53891 \mathrm{D}+02$ & $3.61782 D-08$ & $6.42896 \mathrm{D}-03$ & 59.610 \\
\hline Hst@०3-14 & U235 & Poly & & 1.0005500 & $2.93851 D+02$ & $3.65256 \mathrm{D}-\odot 8$ & $5.92228 \mathrm{D}-\odot 3$ & 58.550 \\
\hline Hst003-15 & U235 & Poly & & ๑. 9990920 & $2.57162 D+02$ & $3.69398 D-08$ & $6.34717 \mathrm{D}-03$ & 64.120 \\
\hline Hst003-16 & U235 & Poly & & $\odot .9988200$ & $3.23827 D+02$ & $4.68287 D-08$ & $1.52737 \mathrm{D}-02$ & 59.230 \\
\hline Hst003-17 & U235 & Poly & & 2400 & $44 D+02$ & $4.54935 \mathrm{D}-\odot 8$ & 1. 42595D-๑2 & 8.880 \\
\hline Hst@03-18 & U235 & Poly & & 0.9963210 & $3.02554 \mathrm{D}+02$ & $8.14354 \mathrm{D}-08$ & $3.77515 \mathrm{D}-02$ & 66.050 \\
\hline Hst००3-19 & U235 & Poly & & 1.0061500 & $2.38232 \mathrm{D}+02$ & $7.52984 \mathrm{D}-\odot 8$ & $3.34843 \mathrm{D}-\odot 2$ & 79.640 \\
\hline HSTO०4 - 1 & U235 & $27 \mathrm{~cm}-\mathrm{D} 20$ & & $\odot .9867170$ & $2.37714 \mathrm{D}+\odot 2$ & $1.84556 \mathrm{D}-05$ & $1.44781 D-01$ & 35.390 \\
\hline HSTO०4 - 2 & U235 & $26 \mathrm{~cm}-\mathrm{D} 20$ & & ๑.9798860 & $2.23644 \mathrm{D}+\odot 2$ & $2.917 \odot 4 \mathrm{D}-06$ & $1.13442 \mathrm{D}-01$ & 35.640 \\
\hline HST००4 - 3 & U235 & $25 \mathrm{~cm}-\mathrm{D} 20$ & & 0.9887620 & $2.05 \odot 23 D+02$ & $5.21246 \mathrm{D}-\odot 7$ & $9.86292 \mathrm{D}-\odot 2$ & 33.360 \\
\hline HSTO०4 - 4 & U235 & $24 \mathrm{~cm}-\mathrm{D} 20$ & & $\odot .9913290$ & $1.94744 \mathrm{D}+02$ & $2.33498 D-07$ & $8.42799 D-02$ & 44.750 \\
\hline 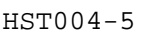 & U235 & $22 \mathrm{~cm}-\mathrm{D} 20$ & & $\odot .9875180$ & $1.91496 \mathrm{D}+02$ & $1.14692 \mathrm{D}-07$ & $7.65492 \mathrm{D}-\odot 2$ & 26.720 \\
\hline HSTO०4 - 6 & U235 & $30 \mathrm{~cm}-\mathrm{D} 20$ & & $\odot .9859550$ & $1.94101 \mathrm{D}+02$ & $7.56469 \mathrm{D}-08$ & $6.61174 \mathrm{D}-02$ & 43.110 \\
\hline HSTO०6 - 1 & U235 & Air & & 0.9780880 & $8.60090 \mathrm{D}+0 \odot$ & $7.42668 D-08$ & $3.24854 \mathrm{D}-02$ & 16.580 \\
\hline HST००6 - 2 & U235 & Air & & 0.9820410 & $7.43396 \mathrm{D}+0 \odot$ & $8.57032 D-08$ & $3.50472 \mathrm{D}-02$ & 11.780 \\
\hline HSTO०6 - 3 & U235 & Air & & $\odot .9962800$ & $6.55088 \mathrm{D}+0 \odot$ & $9.60604 \mathrm{D}-08$ & 4. $09439 D-02$ & 15.920 \\
\hline HSTO०6 - 4 & U235 & Air & & $\odot .9960010$ & $6.03888 \mathrm{D}+00$ & $1.04438 D-07$ & 4. 01437D-02 & 16.390 \\
\hline HSTO०6 - 5 & U235 & Air & & 1.0053000 & $5.52058 \mathrm{D}+\odot \odot$ & $1.140 \odot \odot \mathrm{D}-07$ & $4.47651 \mathrm{D}-02$ & 15.480 \\
\hline HST००6 - 6 & U235 & Air & & $\odot .9890170$ & $5.16055 \mathrm{D}+0 \odot$ & $1.21253 \mathrm{D}-07$ & $4.48398 \mathrm{D}-02$ & 10.800 \\
\hline HST००6 - 7 & U235 & Air & & 1.0022100 & $5.154 \odot 2 D+0 \odot$ & $1.263 \odot 2 \mathrm{D}-07$ & $4.34820 D-02$ & 11.160 \\
\hline HSTO०6 - 8 & U235 & Water & & 0.9815160 & $5.36528 D+01$ & $7.22606 \mathrm{D}-\odot 8$ & $3.28665 \mathrm{D}-02$ & 36.500 \\
\hline HSTO०6 - 9 & U235 & water & & $\odot .9874530$ & $4.17949 \mathrm{D}+01$ & $8.41256 \mathrm{D}-\odot 8$ & $3.59831 \mathrm{D}-02$ & 26.810 \\
\hline
\end{tabular}


HST006-10 U235 HST006-11 U235 HSTO06-12 U235 HSTO06-13 U235 HSTO06-14 U235 HSTO06-15 U235 HST006-16 U235 HSTO06-17 U235 HSTO06-18 U235 HSTO06-19 U235 HSTO०6-20 U235 HSTOO6-21 U235 HST006-22 U235 HST006-23 U235 HSTO06-24 U235 HSTOO6-25 U235 HSTO06-26 U235 HST006-27 U235 HST006-28 U235 HSTO०6-29 U235 HSTOO9-1 U235 HSTO०9-2 U235 HSTOO9-3 U235 HSTO09-4 U235 HSTO10-1 U235 HSTO10-2 U235 HST010-3 U235 HSTO10-4 U235 HST011-1 U235 HSTO11-2 U235 HST012-1 U235 HSTO13-1 U235 HST013-2 U235 HSTO13-3 U235 HSTO13-4 U235 HST014-1 U235 HST014-2 U235 HST014-3 U235 HSTO15-1 U235 HST015-2 U235 HSTO15-3 U235 HST015-4 U235 HSTO15-5 U235 HST016-1 U235 HSTO16-2 U235 HST016-3 U235 HST017-1 U235 HSTO17-2 U235 HST017-3 U235 HST017-4 U235 HST017-5 U235 HST017-6 U235 HST017-7 U235 HSTO17-8 U235 HST018-1 U235 HSTO18-2 U235 HST018-3 U235 HST018-4 U235 HST018-5 U235 HST018-6 U235 HST018-7 U235 HSTO18-8 U235 HSTO18-9 U235 HSTO18-10 U235 HST018-11 U235 HST018-12 U235 HSTO19-1 U235 HSTO19-2 U235 HSTO19-3 U235 HSTO20-1 U235 HSTO20-2 U235
Water

water

Nickel

Nickel

Nickel

Nickel

Nickel

Nickel

$\mathrm{Ni}$-Borated-H2O

$\mathrm{Ni}$-Borated-H2O

$\mathrm{Ni}$-Borated-H2O

$\mathrm{Ni}$-Borated-H2O

Borated-H2O

Borated-H2O

Borated-H2O

Borated-H2O

Borated-H2O

Nickel\&

$\mathrm{H} 2 \mathrm{O}$

Nickel\& $\quad \mathrm{H} 2 \mathrm{O}$

Nickel\& H2O

$\mathrm{H} 2 \mathrm{O}$

$\mathrm{H} 2 \mathrm{O}$

$\mathrm{H} 2 \mathrm{O}$

$\mathrm{H} 2 \mathrm{O}$

$\mathrm{H} 2 \mathrm{O}$

$\mathrm{H} 2 \mathrm{O}$

$\mathrm{H} 2 \mathrm{O}$

$\mathrm{H} 2 \mathrm{O}$

Spherical

Spherical

$\mathrm{H} 2 \mathrm{O}$

ORNL - 1

ORNL - 2

ORNL - 3

ORNL - 4

$\mathrm{H} 2 \mathrm{O}$

$\mathrm{H} 2 \mathrm{O}$

$\mathrm{H} 2 \mathrm{O}$

$\mathrm{H} 2 \mathrm{O}$

$\mathrm{H} 2 \mathrm{O}$

$\mathrm{H} 2 \mathrm{O}$

$\mathrm{H} 20$

$\mathrm{H} 2 \mathrm{O}$

$\mathrm{H} 2 \mathrm{O}$

$\mathrm{H} 2 \mathrm{O}$

$\mathrm{H} 2 \mathrm{O}$

$\mathrm{H} 2 \mathrm{O}$

H2O

$\mathrm{H} 2 \mathrm{O}$

$\mathrm{H} 2 \mathrm{O}$

$\mathrm{H} 2 \mathrm{O}$

$\mathrm{H} 2 \mathrm{O}$

$\mathrm{H} 2 \mathrm{O}$

$\mathrm{H} 2 \mathrm{O}$

$\mathrm{H} 2 \mathrm{O}$

$\mathrm{H} 2 \mathrm{O}$

$\mathrm{H} 2 \mathrm{O}$

$\mathrm{H} 2 \mathrm{O}$

$\mathrm{H} 2 \mathrm{O}$

$\mathrm{H} 2 \mathrm{O}$

$\mathrm{H} 2 \mathrm{O}$

$\mathrm{H} 2 \mathrm{O}$

$\mathrm{H} 2 \mathrm{O}$

$\mathrm{H} 2 \mathrm{O}$

$\mathrm{H} 2 \mathrm{O}$

$\mathrm{H} 2 \mathrm{O}$

$\mathrm{H} 2 \mathrm{O}$

$\mathrm{H} 2 \mathrm{O}$

$\mathrm{H} 2 \mathrm{O}$

Bare

Bare $\odot .99992403 .33610 D+01 \quad 9.44247 D-08 \quad 3.97566 D-02$ $0.99967401 .97892 \mathrm{D}+01$ 1.18460D-07 4.33556D-02 $\odot .98058909 .03099 D+00 \quad 7.45393 D-08 \quad 3.38167 D-02$ $0.98317307 .56454 \mathrm{D}+00 \quad 8.57570 \mathrm{D}-08 \quad 3.63896 \mathrm{D}-02$ $\odot .99562805 .95549 \mathrm{D}+00 \quad 1.05400 \mathrm{D}-07 \quad 3.83561 \mathrm{D}-02$ $1.00619005 .45546 \mathrm{D}+00$ 1.12778D-07 3.98961D-02 $\odot .99371005 .18057 D+001.22635 D-07$ 3.99694D-02 $\odot .99761305 .04715 \mathrm{D}+00$ 1.22727D-07 4.30333D-02 $1.00209008 .30648 \mathrm{D}+00$ 1.00025D-07 4.17711D-02 $1.00879006 .99440 D+0 \odot$ 1.16478D-07 4.21928D-02 $0.992356 \odot 6.73227 \mathrm{D}+00$ 1.21614D-07 4.02699D-02 $1.004430 \odot 6.50114 \mathrm{D}+00$ 1.23835D-07 4.11558D-02 $0.99628301 .03928 \mathrm{D}+01 \quad 9.45091 \mathrm{D}-08$ 4.20301D-02 $\odot .99601109 .08110 D+0 \odot 1.04685 D-07 \quad 4.37219 D-02$ 1.0072200 7.43567D+00 1.14153D-07 3.80843D-02 $\odot .99207106 .93248 D+001.21130 D-07 \quad 4.45596 D-02$ $0.99952906 .70598 D+00 \quad 1.19578 D-07 \quad 4.51193 D-02$ $\odot .98173403 .96895 \mathrm{D}+01 \quad 7.30658 \mathrm{D}-08$ 3.49892D-02 $\odot .98150103 .23283 D+01 \quad 8.63955 D-08$ 3.74185D-02 $1.00495001 .62731 D+01$ 1.21196D-07 4.04188D-02 1.0026900 9.09001D+01 1.18559D-07 5.83525D-02 $1.00515009 .20684 D+01 \quad 9.16985 D-08$ 4.71518D-02 $\begin{array}{llll}1.0032500 & 9.46437 D+01 & 6.69418 D-08 & 2.89287 D-02\end{array}$ $0.99380609 .63655 \mathrm{D}+01 \quad 5.23424 \mathrm{D}-08$ 1.74313D-02 $\odot .99730509 .74780 \mathrm{D}+01 \quad 4.31222 \mathrm{D}-08$ 1. $1.02073 \mathrm{D}-02$ $1.0008000 \quad 9.68630 D+01 \quad 5.00239 D-08$ 9.52100D-03 $\odot .99496609 .63007 \mathrm{D}+01 \quad 7.29906 \mathrm{D}-08$ 9.28316D-03 $0.99387009 .98184 \mathrm{D}+01 \quad 4.04707 \mathrm{D}-08$ 1.04054D-02 $\odot .99899409 .70620 D+01 \quad 3.52086 D-08$ 4.85644D-03 $\begin{array}{llll}1.0039000 & 9.67723 D+01 & 3.49177 D-08 & 4.81387 D-03\end{array}$ $\begin{array}{llll}1.0003000 & 9.87545 D+01 & 3.43999 D-08 & 2.80377 D-03\end{array}$

$\begin{array}{llll}1.0033000 & 6.78337 D+01 & 3.22586 D-08 & 2.91948 D-03\end{array}$

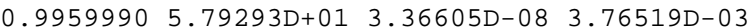
$\odot .99428505 .04869 D+01 \quad 3.39834 D-08 \quad 3.26998 D-03$ $\begin{array}{llll}1.0007900 & 4.79854 D+01 & 3.42788 D-08 & 2.64796 D-03\end{array}$ $\begin{array}{lllll}1.9914580 & 6.23600 D+01 & 3.72222 D-08 & 7.73156 D-03\end{array}$ $\begin{array}{llll}1.0117500 & 5.96659 D+01 & 3.81083 D-08 & 7.72238 D-03\end{array}$ $\begin{array}{llll}1.0219800 & 4.94480 \mathrm{D}+01 & 3.86968 \mathrm{D}-08 & 7.89727 \mathrm{D}-03\end{array}$ $0.99498403 .65892 D+01 \quad 4.06761 D-08$ 1.16348D-02 $\odot .98340406 .07636 \mathrm{D}+01$ 4.05407D-08 1.02116D-02 $1.00361002 .84040 D+01 \quad 4.28928 D-08$ 1.27669D-02 $1.00932004 .74908 D+01 \quad 4.24567 D-08$ 1.13708D-02 $\begin{array}{llll}1.0118300 & 3.88200 \mathrm{D}+01 & 4.42498 \mathrm{D}-08 & 1.21221 \mathrm{D}-02\end{array}$ $0.99335204 .91735 \mathrm{D}+01 \quad 4.66646 \mathrm{D}-08$ 1.66782D-02 $\begin{array}{llll}1.013200 \odot & 4.59306 \mathrm{D}+01 & 4.75663 \mathrm{D}-08 & 1.92624 \mathrm{D}-02\end{array}$ $\begin{array}{llll}1.0305000 & 3.83231 D+01 & 5.12785 D-08 & 1.61616 D-02\end{array}$ $\odot .98623406 .53191 \mathrm{D}+01 \quad 5.21368 \mathrm{D}-08$ 2.15156D-02 $0.98046903 .14207 \mathrm{D}+01 \quad 5.38357 \mathrm{D}-08$ 2.11802D-02 $\odot .9813800 \quad 4.81117 D+01 \quad 5.23203 D-08$ 2.05293D-02 $\odot .99735306 .92974 D+01 \quad 5.42230 D-08$ 1.98074D-02 $1.00614006 .46168 \mathrm{D}+01 \quad 5.61486 \mathrm{D}-08$ 1.83663D-02

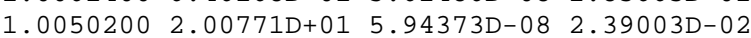
$\begin{array}{llll}1.0082900 & 4.73090 D+01 & 5.76350 D-08 & 2.23988 D-02\end{array}$ $\begin{array}{llll}1.0054700 & 1.56990 \mathrm{D}+01 & 6.21163 \mathrm{D}-08 & 2.33366 \mathrm{D}-02\end{array}$ $0.98429406 .46671 D+01 \quad 6.46793 D-08$ 2.84241D-02 $0.98228702 .89813 \mathrm{D}+01$ 7.00215D-08 3.49104D-02 $0.98320004 .47903 \mathrm{D}+01 \quad 6.65453 \mathrm{D}-08$ 3.18663D-02 $\odot .99864307 .28654 D+01 \quad 7.03436 D-08$ 3.15005D-02 $\odot .99680602 .11190 \mathrm{D}+01 \quad 7.86145 \mathrm{D}-08$ 3.26466D-02 $\odot .98789303 .92065 \mathrm{D}+01 \quad 7.43952 \mathrm{D}-08$ 3.28853D-02 $\begin{array}{llll}1.0068600 & 5.98366 \mathrm{D}+01 & 7.49936 \mathrm{D}-08 & 2.76262 \mathrm{D}-02\end{array}$ $1.02118001 .60728 D+01 \quad 8.47065 D-08 \quad 3.60930 D-02$ $1.00492003 .74639 \mathrm{D}+01 \quad 8.10074 \mathrm{D}-08$ 3.17456D-02 $1.02427001 .21814 \mathrm{D}+01$ 9.27956D-08 3.49867D-02 $1.02022003 .79053 D+01 \quad 8.52494 D-08$ 3.35101D-02 1.0178500 3.04432D+01 9.51963D-08 3.65045D-02 $\odot .99316206 .20547 D+01$ 8.93378D-08 4.20653D-02 $1.00355006 .63952 D+01 \quad 8.87593 D-08$ 3.94742D-02 $1.00439006 .35064 D+01$ 1.01554D-07 4.23370D-02 $\odot .99316301 .16950 D+01 \quad 3.30102 D-07 \quad 7.29916 D-02$ $\begin{array}{llll}1.0034600 & 2.17529 D+01 & 1.28352 D-07 & 6.50870 D-02\end{array}$
21.550

13.550

23.560

15.030

13.770

10.470

10.610

12.380

11.840

15.190

13.550

13.580

11.700

17.470

12.610

14.050

11.030

26.770

28.330

13.840

44.470

44.700

42.380

53.690

53.060

47.470

39.530

53.120

34.580

51.340

33.580

22.910

24.050

22.480

20.200

37.470

45.030

39.050

31.080

33.880

28.060

39.770

42.860

34.450

23.700

35.670

43.170

29.810

26.750

64.670

56.590

39.840

39.480

35.390

32.880

32.160

39.050

56.020

34.610

43.060

32.280

18.280

35.840

23.750

28.190

21.480

41.690

43.360

42.420

27.330

34.700 


\begin{tabular}{|c|c|c|}
\hline HST०20-3 & U235 & Bare \\
\hline HST०2О- 4 & U235 & Bare \\
\hline HST०2०- 5 & U235 & Bare \\
\hline Hst-25-1 & U235 & Water \\
\hline Hst@25-2 & U235 & water \\
\hline Hst025-3 & U235 & Water \\
\hline Hst@25-4 & U235 & Water \\
\hline Hste25-5 & U235 & water \\
\hline Hst025-6 & U235 & Water \\
\hline Hst025-7 & U235 & Water \\
\hline Hst025-8 & U235 & Water \\
\hline Hst@25-9 & U235 & water \\
\hline Hste25-10 & U235 & Water \\
\hline Hst $\odot 25-11$ & U235 & Water \\
\hline Hst $\odot 25-12$ & U235 & Water \\
\hline Hste25-13 & U235 & Water \\
\hline Hst-25-14 & U235 & Water \\
\hline Hst025-15 & U235 & Water \\
\hline Hst@25-16 & U235 & Water \\
\hline Hst-25-17 & U235 & Water \\
\hline Hste25-18 & U235 & Water \\
\hline Hst०27-1 & U235 & Bare \\
\hline Hst@27-2 & U235 & B4C-rod \\
\hline Hst027-3 & U235 & B4C-rod \\
\hline Hst $027-4$ & U235 & B4C-rod \\
\hline Hst $027-5$ & U235 & B4C-rod \\
\hline Hst027-6 & U235 & Cd-rod \\
\hline Hst@27-7 & U235 & Cd-rod \\
\hline Hst@27-8 & U235 & Cd-rod \\
\hline Hst@27-9 & U235 & Cd-rod \\
\hline Hst@28-1 & U235 & Water \\
\hline Hst@28-2 & U235 & Water \\
\hline Hst028-3 & U235 & Water \\
\hline Hst@28-4 & U235 & Water \\
\hline Hst028-5 & U235 & Water \\
\hline Hst028-6 & U235 & Water \\
\hline Hst028-7 & U235 & Water \\
\hline Hst@28-8 & U235 & Water \\
\hline Hst@28-9 & U235 & Water \\
\hline Hst $028-10$ & U235 & Water \\
\hline Hst $028-11$ & U235 & Water \\
\hline Hst $028-12$ & U235 & Water \\
\hline Hst $028-13$ & U235 & Water \\
\hline Hst $028-14$ & U235 & Water \\
\hline Hst028-15 & U235 & Water \\
\hline Hst $028-16$ & U235 & Water \\
\hline Hst $028-17$ & U235 & Water \\
\hline Hst@28-18 & U235 & Water \\
\hline Hst029-1 & U235 & Water \\
\hline Hst029-2 & U235 & Water \\
\hline HSTO29-3 & U235 & Water \\
\hline Hst@29-4 & U235 & water \\
\hline Hst-29-5 & U235 & Water \\
\hline Hst@29-6 & U235 & Water \\
\hline Hst029-7 & U235 & Water \\
\hline Hst030-1 & U235 & Water \\
\hline Hst $030-2$ & U235 & Water \\
\hline Hst030-3 & U235 & Water \\
\hline Hst@3०-4 & U235 & Water \\
\hline Hst $030-5$ & U235 & Water \\
\hline Hst030-6 & U235 & Water \\
\hline Hst@30-7 & U235 & Water \\
\hline HST०32 - 1 & U235 & ORNL - 10 \\
\hline HST०42-1 & U235 & solution \\
\hline HST०42 - 2 & U235 & solution \\
\hline HST०42-3 & U235 & solution \\
\hline HST๑42 - 4 & U235 & solution \\
\hline HST०42- 5 & U235 & solution \\
\hline HSTO42 - 6 & U235 & solution \\
\hline HST๑42- 7 & U235 & solution \\
\hline HST०42 - 8 & U235 & solution \\
\hline
\end{tabular}

$\begin{array}{llll}1.0107300 & 4.59778 D+01 & 6.45982 D-08 & 6.18390 D-02\end{array}$ $\begin{array}{llll}1.0111400 & 4.59874 D+01 & 6.49117 D-08 & 6.14391 D-02\end{array}$ $1.01813001 .08422 \mathrm{D}+02$ 4.26071D-08 5.28608D-02 $1.00323007 .20551 D+01 \quad 3.52280 D-08$ 6.19672D-03 1. $00401006.40292 D+01 \quad 3.54304 D-08$ 5.97240D-03 $0.9955010 \quad 5.61008 \mathrm{D}+01 \quad 3.63341 \mathrm{D}-08$ 7.59233D-03 $0.99747106 .05346 \mathrm{D}+01 \quad 3.54637 \mathrm{D}-08$ 6.83621D-03 $1.00282005 .61312 \mathrm{D}+01 \quad 3.82290 \mathrm{D}-08$ 8.92724D-03 $\begin{array}{llll}1.0100200 & 5.31160 D+01 & 3.61138 D-08 & 5.87278 D-03\end{array}$ $\begin{array}{llll}1.0186600 & 5.03002 \mathrm{D}+01 & 3.75746 \mathrm{D}-08 & 6.82962 \mathrm{D}-03\end{array}$ $\begin{array}{llll}1.0093000 & 4.84594 D+01 & 3.84963 D-08 & 8.01624 D-03\end{array}$ $1.01020004 .78196 \mathrm{D}+01$ 4.08044D-08 9.88976D-03 $1.01245004 .46683 \mathrm{D}+01 \quad 4.53058 \mathrm{D}-08$ 1.24644D-02 $1.01021004 .12499 \mathrm{D}+01 \quad 4.58003 \mathrm{D}-08$ 1.11030D-02 $1.00629004 .13196 \mathrm{D}+01$ 5.02960D-08 1.63532D-02 $1.02362003 .94636 \mathrm{D}+01 \quad 5.04541 \mathrm{D}-08$ 1.53409D-02 $\begin{array}{llll}1.0124200 & 3.80767 D+01 & 5.71455 D-08 & 2.14752 D-02\end{array}$ $\begin{array}{llll}1.0041700 & 3.80199 D+01 & 5.59860 D-08 & 2.11287 D-02\end{array}$ $\begin{array}{llll}1.0217300 & 4.02640 D+01 & 6.89236 D-08 & 2.92639 D-02\end{array}$ $1.01266003 .90404 \mathrm{D}+01$ 6.64184D-08 2.76005D-02 $\begin{array}{llll}1.0048400 & 3.92136 D+01 & 6.28921 D-08 & 2.41676 D-02\end{array}$ $0.99099701 .17912 \mathrm{D}+01 \quad 4.55944 \mathrm{D}-08$ 1.59794D-02 $\odot .98938001 .13896 \mathrm{D}+01$ 4.51901D-08 1.59979D-02 $0.99511301 .13564 \mathrm{D}+01 \quad 4.52787 \mathrm{D}-08$ 1.55106D-02 $\odot .99856801 .11142 \mathrm{D}+01 \quad 4.54605 \mathrm{D}-08$ 1.49291D-02 $0.99946401 .09716 \mathrm{D}+01 \quad 4.55748 \mathrm{D}-08 \mathrm{1} \quad 1.62375 \mathrm{D}-02$ $\odot .98632001 .18193 D+014.54639 D-08$ 1.50358D-02 $0.99585301 .24214 D+014.52285 D-08$ 1.56656D-02 $1.00331001 .39149 \mathrm{D}+01$ 4.55261D-08 1.52562D-02 $0.99592801 .53247 \mathrm{D}+01$ 4.55801D-08 1.55371D-02 $0.9909770 \quad 9.74871 \mathrm{D}+01 \quad 3.78023 \mathrm{D}-08 \quad 7.70982 \mathrm{D}-03$ $0.9993170 \quad 8.50264 D+01 \quad 3.76153 D-08$ 7.96090D-03 $0.99515609 .78164 \mathrm{D}+01 \quad 3.80521 \mathrm{D}-08$ 8.12055D-03 $\begin{array}{llll}1.0034200 & 8.62099 D+01 & 3.75270 D-08 & 6.72069 D-03\end{array}$ $0.99407009 .92178 D+01 \quad 3.78100 D-08 \quad 7.85110 D-03$ $\begin{array}{llll}1.0001200 & 8.74663 D+01 & 3.76502 D-08 & 8.38606 D-03\end{array}$ $\odot .99831101 .02059 \mathrm{D}+02 \quad 3.77691 \mathrm{D}-08 \quad 7.68294 \mathrm{D}-03$ $\odot .9955870 \quad 9.28300 \mathrm{D}+01 \quad 3.77991 \mathrm{D}-08$ 8.02536D-03 $\odot .9965070 \quad 9.42574 D+01 \quad 6.13550 D-08$ 2.79159D-02 $0.9882550 \quad 8.37454 D+01 \quad 6.16888 D-08$ 2.68364D-02 $1.0004800 \quad 9.48160 D+01 \quad 6.18928 D-08$ 2.80993D-02 $\odot .9905700 \quad 8.49440 \mathrm{D}+01 \quad 6.17872 \mathrm{D}-08 \quad 2.71342 \mathrm{D}-02$ $\odot .99887809 .63235 \mathrm{D}+01 \quad 6.21142 \mathrm{D}-08$ 2.74747D-02 $0.99239808 .67965 \mathrm{D}+01 \quad 6.28908 \mathrm{D}-08$ 2.72688D-02 $\begin{array}{llll}1.0006600 & 9.71066 \mathrm{D}+01 & 6.27378 D-08 & 2.86713 \mathrm{D}-02\end{array}$ 0.9965600 8.84665D+01 $6.28724 \mathrm{D}-08$ 2.90025D-02 $\odot .99545501 .00050 D+02 \quad 6.37737 D-08 \quad 2.80016 D-02$ $0.99620309 .18485 \mathrm{D}+01 \quad 6.38338 \mathrm{D}-08$ 2.82808D-02 $\odot .99451506 .43533 \mathrm{D}+01 \quad 6.37726 \mathrm{D}-08 \quad 2.84282 \mathrm{D}-02$ $\odot .99763907 .70867 D+01 \quad 6.30460 D-08 \quad 2.88270 D-02$ $\odot .98951707 .51473 D+01 \quad 6.35847 D-08 \quad 2.65266 D-02$ $0.98994506 .83519 \mathrm{D}+01 \quad 6.46514 \mathrm{D}-08$ 3.11837D-02 $0.99410906 .61324 D+01 \quad 6.49016 D-08 \quad 3.16371 D-02$ $\odot .99358306 .67541 \mathrm{D}+01$ 6.52923D-08 2.84098D-02 $0.99793806 .85722 \mathrm{D}+01 \quad 6.50794 \mathrm{D}-08$ 2.90039D-02 $0.99484907 .58955 \mathrm{D}+01 \quad 3.77498 \mathrm{D}-08$ 7.97843D-03 $\odot .9963870 \quad 8.01774 \mathrm{D}+01 \quad 3.82600 \mathrm{D}-08$ 9.53785D-03

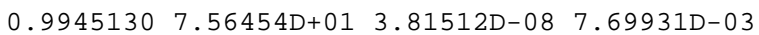
$1.00202006 .31781 D+01 \quad 6.46468 D-08$ 2.86686D-02 $0.99193808 .14139 D+01 \quad 6.46069 D-08$ 3.07526D-02 $0.99525707 .81088 D+01 \quad 6.34704 D-08 \quad 2.68232 D-02$ $\odot .99414807 .06326 \mathrm{D}+01 \quad 6.48608 \mathrm{D}-08$ 2.99802D-02 $\begin{array}{llll}1.0050700 & 8.99774 \mathrm{D}+01 & 3.10666 \mathrm{D}-08 & 2.74723 \mathrm{D}-03\end{array}$ $0.9956920 \quad 8.39753 \mathrm{D}+01 \quad 3.21981 \mathrm{D}-08$ 2.65125D-03 $0.99933508 .50725 \mathrm{D}+01 \quad 3.19964 \mathrm{D}-08$ 2.90555D-03 $0.9978060 \quad 9.16924 D+01 \quad 3.12973 D-08 \quad 2.42175 D-03$ $\begin{array}{llll}1.0035400 & 9.53465 D+01 & 3.09090 D-08 & 3.39757 D-03\end{array}$ $0.9970630 \quad 9.75885 D+01 \quad 3.13080 D-08 \quad 2.70971 D-03$ $\odot .9975150 \quad 9.86487 D+01 \quad 3.11927 D-08$ 1.45421D-03 $0.9990650 \quad 9.97597 D+01 \quad 3.10917 D-08 \quad 2.47847 D-03$ $\odot .99862801 .01131 D+02 \quad 3.11834 D-08$ 1.18062D-03
31.620 35.730 35.860 34.970 43.280 54.590 73.840 51.420 53.750 42.380 37.390 43.500 46.840 31.910 31.170 31.000 34.620 37.170 25.620 26.620 32.330 27.480 20.700 32.230 21.250 26.060 28.330 28.380 31.410 27.120 60.690 51.670 39.750 61.450 41.500 42.270 62.970 59.920 39.720 50.970 60.390

61.390

59.440 69.000 57.700 57.300 80.300 86.110 58.910 92.310 73.840 86.890 85.390 85.890 77.410 62.230 84.720 72.060 64.270 85.050 89.060 96.310 51.700 37.750 37.980 41.950 41.550 43.770 41.660 43.050 42.120 
HSTO43-1 U235 HSTO43-2 U235 HSTO43-3 U235 ISTO04-1 U235 LCTOO6-1 U235 LCTOO6-2 U235 LCTO०6-3 U235 LCTO०6-4 U235 LCTO०6-5 U235 LCTO०6-6 U235 LCTO०6-7 U235 LCTO०6-8 U235 LCTO०6-9 U235 LCTO०6-10 U235 LCT006-11 U235 LCTO०6-12 U235 LCTO०6-13 U235 LCT०06-14 U235 LCTO०6-15 U235 LCT006-16 U235 LCTOO6-17 U235 LCT006-18 U235 LCT033-01 U235 LCTO33-02 U235 LCT033-03 U235 LCTO33-๑4 U235 LCT033-05 U235 LCTO33-06 U235 LCT033-07 U235 LCT033-08 U235 LCT०33-09 U235 LCTO33-10 U235 LCT033-11 U235 LCTO33-12 U235 LCTO33-13 U235 LCT033-14 U235 LCTO33-15 U235 LCT033-16 U235 LCTO33-17 U235 LCT033-18 U235 LCT033-19 U235 LCT033-20 U235 LCT033-21 U235 LCT033-22 U235 LCTO33-23 U235 LCTO33-24 U235 LCTO33-25 U235 LCTO33-26 U235 LCT033-27 U235 LCTO33-28 U235 LCT033-29 U235 LCT०33-30 U235 LCT033-31 U235 LCTO33-32 U235 LCTO33-33 U235 LCTO33-34 U235 LCT033-35 U235 LCT033-36 U235 LCTO33-37 U235 LCTO33-38 U235 LCTO33-39 U235 LCT033-40 U235 LCTO33-41 U235 LCT033-42 U235 LCTO33-43 U235 LCTO33-44 U235 LCT033-45 U235 LCTO33-46 U235 LCT033-47 U235 LCTO33-48 U235 LCTO33-49 U235 solution

solution

solution

$\mathrm{BeO}$

water

water

water

Water

water

water

water

water

water

water

Water

water

Water

Water

Water

water

water

Water

Paraffin+Plexi Paraffin+Plexi Paraffin+Plexi Paraffin+Plexi Paraffin+Plexi Paraffin+Plexi Paraffin+Plexi Paraffin+Plexi Paraffin+Plexi Paraffin+Plexi Paraffin+Plexi Paraffin+Plexi Polyethy+Plexi Polyethy+Plexi Polyethy+Plexi Polyethy+Plexi Paraffin+Plexi Paraffin+Plexi Paraffin+Plexi Paraffin+Plexi Paraffin+Plexi Polyethy+Plexi Bare

Bare

Bare

Bare

Bare

Bare

Bare

Bare

Bare

Bare

Bare

Bare

Bare

Bare

Bare

Bare

Bare

Bare

Bare

Bare

Bare

Bare

Bare

Bare

Bare

Bare

Bare $\odot .98858801 .00808 D+01 \quad 4.52120 D-08$ 1.44159D- 02 $\begin{array}{llll}1.0046200 & 5.46361 D+01 & 3.26583 D-08 & 3.04923 D-03\end{array}$ $\begin{array}{lllll}1.0004200 & 6.83683 D+01 & 3.20313 D-08 & 3.03902 D-03\end{array}$ $\begin{array}{llll}1.0094600 & 3.32817 D+02 & 3.77423 D-08 & 2.97943 D-01\end{array}$ $\odot .99645807 .04995 D+01 \quad 5.39703 D-08 \quad 2.22928 D-01$ $0.99718506 .85257 \mathrm{D}+01 \quad 5.46611 \mathrm{D}-08$ 2.22522D-01 $\odot .9987370 \quad 6.62960 D+01 \quad 5.50067 D-08$ 2.25059D-01 $1.00300007 .33820 D+01$ 5.05258D-08 1.99915D-01 $\odot .99403407 .23227 D+01 \quad 5.04767 D-08$ 2.02711D-01 $0.99931306 .95500 \mathrm{D}+01 \quad 5.09578 \mathrm{D}-08 \quad 2.00324 \mathrm{D}-01$ $\begin{array}{llll}1.0010000 & 6.63711 D+01 & 5.17920 D-08 & 2.07533 D-01\end{array}$ $\begin{array}{llll}1.0022900 & 6.63091 D+01 & 5.17372 D-08 & 2.06706 D-01\end{array}$ $\odot .99390007 .58085 \mathrm{D}+01 \quad 4.71205 \mathrm{D}-08$ 1.70237D-01 $1.00314007 .34542 \mathrm{D}+01 \quad 4.69748 \mathrm{D}-08$ 1.70814D-01 $0.99898607 .21817 \mathrm{D}+01$ 4.73005D-08 1.70917D-01 $\odot .99475607 .04392 D+01 \quad 4.74811 D-08$ 1.83109D-01 $0.99245406 .87898 \mathrm{D}+01 \quad 4.84709 \mathrm{D}-08$ 1.81479D-01 $0.99426707 .97166 \mathrm{D}+01$ 4.47955D-08 1.54971D-01 $0.99946107 .64146 D+01 \quad 4.50831 D-08$ 1.50811D-01 $0.99763207 .46951 D+01 \quad 4.53475 D-08$ 1.62719D-01 $0.99972707 .29280 \mathrm{D}+01 \quad 4.59349 \mathrm{D}-08$ 1.62423D-01 $1.00241007 .13445 \mathrm{D}+01 \quad 4.56744 \mathrm{D}-08$ 1.53206D-01 $\odot .99671604 .58247 D+01 \quad 5.57755 D-08$ 1.98449D-01 $0.99752404 .57577 D+01 \quad 5.65846 D-08$ 1.95478D-01 $\odot .99979804 .62208 D+01 \quad 5.55188 D-08$ 1.81620D-01 $\begin{array}{llll}1.0008800 & 4.68781 D+01 & 5.53347 D-08 & 1.97528 D-01\end{array}$ $1.00787005 .18562 \mathrm{D}+01 \quad 4.73898 \mathrm{D}-08$ 1.53346D-01 $1.00009005 .24806 \mathrm{D}+01 \quad 4.80938 \mathrm{D}-08$ 1.57149D-01 $0.9999600 \quad 5.39184 \mathrm{D}+01 \quad 4.76171 \mathrm{D}-08$ 1.51377D-01 $0.99892505 .76158 D+01 \quad 4.26845 D-08$ 1.17148D-01 $1.00267005 .64838 D+01 \quad 4.29847 D-08$ 1.22744D-01 $0.99460905 .88413 D+01 \quad 4.07995 D-08$ 1.07655D-01 $\odot .99104905 .98136 \mathrm{D}+01 \quad 4.12056 \mathrm{D}-08$ 1.07435D-01 $\odot .99520406 .04032 \mathrm{D}+01 \quad 4.12522 \mathrm{D}-08$ 1.09230D-01 $0.9951770 \quad 6.22721 \mathrm{D}+01 \quad 3.94744 \mathrm{D}-08$ 9.89822D-02 $\odot .98868906 .54552 \mathrm{D}+01 \quad 3.69871 \mathrm{D}-08 \quad 7.79377 \mathrm{D}-02$ $\odot .9962560 \quad 6.47028 \mathrm{D}+01 \quad 3.72445 \mathrm{D}-08 \quad 7.41791 \mathrm{D}-02$ $0.99406606 .47546 \mathrm{D}+01 \quad 3.68568 \mathrm{D}-08$ 6.73517D-02 $1.01059004 .91960 D+01 \quad 6.31551 D-08$ 1.81469D-01 $\begin{array}{llll}1.0049700 & 5.02123 D+01 & 6.25682 D-08 & 1.85066 D-01\end{array}$ $1.01769004 .96665 D+01 \quad 6.37498 D-08$ 1.81470D-01 $\begin{array}{llll}1.0130300 & 5.14283 D+01 & 6.28683 D-08 & 1.80065 D-01\end{array}$ $\begin{array}{lll}1.00894005 .16301 D+01 & 6.30126 D-08 & 1.80703 D-01\end{array}$ $1.01501006 .10877 D+01 \quad 4.65026 D-08$ 1.14286D-01 $0.99709101 .94170 D+01 \quad 5.94178 D-08 \quad 2.16333 D-01$ $\odot .99832401 .94636 \mathrm{D}+01$ 5.94887D-08 2.17384D-01 $\odot .99652701 .93099 D+01 \quad 5.90043 D-08 \quad 2.18705 D-01$ $1.00488002 .36369 D+01 \quad 4.91903 D-08$ 1.67700D-01 $\begin{array}{llll}1.0014100 & 2.34557 D+01 & 4.94179 D-08 & 1.71467 D-01\end{array}$ $\odot .99857902 .34145 \mathrm{D}+01 \quad 4.89737 \mathrm{D}-08$ 1.63872D-01 $1.00001002 .34125 \mathrm{D}+01 \quad 4.88537 \mathrm{D}-08$ 1.68127D-01 $\odot .99184902 .79416 \mathrm{D}+01$ 4.40999D- 08 1.37301D-01 $0.99435902 .79290 D+01 \quad 4.41356 D-08$ 1.35248D-01 $\odot .99508502 .79510 D+01 \quad 4.44745 D-08$ 1.39671D-01 $0.99259102 .79564 D+01 \quad 4.44449 D-08$ 1.42773D-01 $\odot .99587802 .80419 D+01 \quad 4.42227 D-08$ 1.36102D-01 $0.99701603 .16941 \mathrm{D}+01 \quad 4.15482 \mathrm{D}-08$ 1.09101D-01 1. $00062003.19770 D+01 \quad 4.21204 D-08$ 1.14921D-01 $1.00068003 .19275 \mathrm{D}+01 \quad 4.17182 \mathrm{D}-08$ 1.26861D-01 $0.9876920 \quad 3.13563 D+01 \quad 4.22773 D-08$ 1.23369D-01 $0.99494103 .18371 D+01 \quad 4.16562 D-08$ 1.14786D-01 $0.99250703 .16646 \mathrm{D}+01 \quad 4.15722 \mathrm{D}-08$ 1.10535D-01 $\odot .99189803 .61746 D+01$ 4.02907D-08 1.05375D-01 $0.99958603 .66962 \mathrm{D}+01 \quad 3.97128 \mathrm{D}-08$ 9.89199D-02 $\odot .99371603 .63175 D+014.01666 D-08$ 1.05238D-01 $0.98800105 .03229 D+01 \quad 3.69600 D-08$ 6.94654D-02 $\odot .98865805 .08394 \mathrm{D}+01 \quad 3.71853 \mathrm{D}-08$ 7.55636D-02 $0.99476605 .07794 D+01 \quad 3.69135 D-08$ 7.22529D-02 $\begin{array}{llll}1.0097300 & 1.32566 \mathrm{D}+01 & 7.11852 \mathrm{D}-08 & 2.07737 \mathrm{D}-01\end{array}$ $\begin{array}{llll}1.0154700 & 1.32355 D+01 & 7.12131 D-08 & 2.06926 D-01\end{array}$ $1.01106001 .32390 D+01 \quad 7.14592 D-08$ 2.11353D-01
137.910 350.610 300.940 100.270

53.440

50.810

49.060

35.810

47.270

53.500

33.330

49.700

53.310

42.890

46.690

61.480

45.520

51.910

50.050

44.880

50.920

34.280

27.780

27.170

19.140

31.220

27.440

22.190

32.380

29.770

34.560

37.090

28.840

30.720

36.470

26.420

28.060

24.670

46.620

33.590

30.380

37.620

36.700

26.310

20.170

23.160

18.360

25.230

20.590

24.110

23.110

21.110

16.020

24.500

15.860

26.160

17.080

19.720

23.200

19.020

25.000

23.580

23.970

26.120

32.080

25.090

23.270

26.360

13.280

20.410

28.060 


\begin{tabular}{|c|c|c|}
\hline LCT०33-50 & U235 & Bare \\
\hline LCTO33-51 & U235 & Bare \\
\hline LСT०33-52 & U235 & Bare \\
\hline LMT००1 & U-nat & D20 \\
\hline 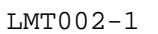 & U235 & D20 \\
\hline 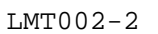 & U235 & D20 \\
\hline LMTO०2 - 3 & U235 & D20 \\
\hline 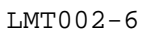 & U235 & D20 \\
\hline LMTO०2-10 & U235 & D20 \\
\hline LMT००2 - 11 & U235 & D20 \\
\hline LMT००2 - 12 & U235 & D20 \\
\hline LSTO०1 & U235 & Sheba-11 \\
\hline LSTOO2 - 1 & U235 & $15 \mathrm{~cm}-\mathrm{H} 20$ \\
\hline LSTO०2 - 2 & U235 & Bare \\
\hline LSTO०2 - 3 & U235 & $15 \mathrm{~cm}-\mathrm{H} 20$ \\
\hline LSTO०3 - 1 & U235 & Bare \\
\hline LSTO०3-2 & U235 & Bare \\
\hline LSTOO3 - 3 & U235 & Bare \\
\hline LSTO०3- 4 & U235 & Bare \\
\hline LSTO०3 - 5 & U235 & Bare \\
\hline LSTO०3- 6 & U235 & Bare \\
\hline LSTO०3 - 7 & U235 & Bare \\
\hline LSTO०3- 8 & U235 & Bare \\
\hline LSTO०3-9 & U235 & Bare \\
\hline LSTO०4 - 1 & U235 & $30 \mathrm{~cm}-\mathrm{H} 20$ \\
\hline LSTO०4-2 & U235 & $30 \mathrm{~cm}-\mathrm{H} 20$ \\
\hline LSTO०4-3 & U235 & $30 \mathrm{~cm}-\mathrm{H} 20$ \\
\hline LSTO९4 - 4 & U235 & $30 \mathrm{~cm}-\mathrm{H} 20$ \\
\hline LSTO०4- 5 & U235 & $30 \mathrm{~cm}-\mathrm{H} 20$ \\
\hline LSTO०4- 6 & U235 & $30 \mathrm{~cm}-\mathrm{H} 20$ \\
\hline LSTO९4 - 7 & U235 & $30 \mathrm{~cm}-\mathrm{H} 20$ \\
\hline LSTO०5- 1 & U235 & $\mathrm{H} 2 \mathrm{O}$ \\
\hline LSTOO5 - 2 & U235 & $\mathrm{H} 2 \mathrm{O}$ \\
\hline LSTO०5 - 3 & U235 & $\mathrm{H} 2 \mathrm{O}$ \\
\hline LSTOO7 - 1 & U235 & Bare \\
\hline LSTO०7 - 2 & U235 & Bare \\
\hline LSTO०7 - 3 & U235 & Bare \\
\hline LSTO०7 - 4 & U235 & Bare \\
\hline LSTO०7-5 & U235 & Bare \\
\hline LSTO०9-1 & U235 & Concrete \\
\hline LSTO०৪- 1 & U235 & Concrete \\
\hline LSTO৫৪- 2 & U235 & Concrete \\
\hline LSTO०8-3 & U235 & Concrete \\
\hline LSTO०8-4 & U235 & Concrete \\
\hline LST००9- 2 & U235 & Concrete \\
\hline LSTO०9-3 & U235 & Concrete \\
\hline LST010-1 & U235 & Poly \\
\hline LST010-2 & U235 & Poly \\
\hline LSTO10 - 3 & U235 & Poly \\
\hline LST010 - 4 & U235 & Poly \\
\hline LST016 - 1 & U235 & $30 \mathrm{~cm}-\mathrm{H} 20$ \\
\hline LSTO16 - 2 & U235 & $30 \mathrm{~cm}-\mathrm{H} 2 \odot$ \\
\hline LSTO16 - 3 & U235 & $30 \mathrm{~cm}-\mathrm{H} 20$ \\
\hline LSTO16 - 4 & U235 & $30 \mathrm{~cm}-\mathrm{H} 20$ \\
\hline LST016 - 5 & U235 & $30 \mathrm{~cm}-\mathrm{H} 20$ \\
\hline LST016 - 6 & U235 & $30 \mathrm{~cm}-\mathrm{H} 20$ \\
\hline LST016 - 7 & U235 & $30 \mathrm{~cm}-\mathrm{H} 20$ \\
\hline LSTO17 - 1 & U235 & Bare \\
\hline LST017 - 2 & U235 & Bare \\
\hline LST017-3 & U235 & Bare \\
\hline LSTO17 - 4 & U235 & Bare \\
\hline LST017 - 5 & U235 & Bare \\
\hline LST017- 6 & U235 & Bare \\
\hline LST018-1 & U235 & concrete \\
\hline LST018 - 2 & U235 & concrete \\
\hline LST018- 3 & U235 & concrete \\
\hline LSTO18 - 4 & U235 & concrete \\
\hline LST018- 5 & U235 & concrete \\
\hline LST018 - 6 & U235 & concrete \\
\hline LSTO19-1 & U235 & Poly \\
\hline LST019-2 & U235 & Poly \\
\hline
\end{tabular}

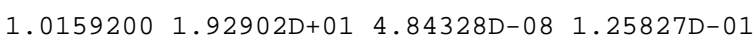
$1.00700001 .90832 D+01 \quad 4.85885 D-08$ 1.23934D-01 $1.00962001 .91175 D+01 \quad 4.89552 D-08$ 1.23961D-01 $\begin{array}{llll}1.0043800 & 4.62799 D+02 & 4.26519 D-08 & 2.26960 D-01\end{array}$ $1.01428001 .19955 D+03 \quad 3.67893 D-08$ 1.13881D-01 $\begin{array}{llll}1.0034100 & 1.00522 D+03 & 3.79872 D-08 & 1.20027 D-01\end{array}$ $\begin{array}{llll}1.0143100 & 1.07359 D+03 & 3.72924 D-08 & 1.15740 D-01\end{array}$ $1.00074001 .12641 \mathrm{D}+03$ 4.52067D-08 $1.35732 \mathrm{D}-01$ $1.00142008 .42914 D+02 \quad 3.91188 D-08$ 1.20068D-01 $1.00034008 .23488 D+02 \quad 4.03968 D-08$ 1.19419D-01 $\begin{array}{llll}1.0108700 & 9.74173 D+02 & 3.79524 D-08 & 1.20526 D-01\end{array}$ $\begin{array}{llll}1.0159700 & 2.77872 \mathrm{D}+01 & 3.83162 \mathrm{D}-08 & 5.23972 \mathrm{D}-02\end{array}$ $0.99703908 .44994 \mathrm{D}+01 \quad 3.30005 \mathrm{D}-08$ 3.13422D-02 $\odot .996550 \odot 5.23548 \mathrm{D}+01 \quad 3.32932 \mathrm{D}-\odot 8 \quad 3.20273 \mathrm{D}-02$ $\begin{array}{llll}1.0084300 & 8.67595 D+01 & 3.31580 D-08 & 3.15814 D-02\end{array}$ $\odot .9941920 \quad 4.24241 D+01 \quad 3.49932 D-08$ 2.11901D-02 $\begin{array}{llll}1.0021100 & 4.79347 D+01 & 3.39918 D-08 & 1.91476 D-02\end{array}$ $\begin{array}{lllll}1.0004300 & 4.83505 D+01 & 3.43638 D-08 & 2.01019 D-02\end{array}$ $\odot .99296605 .04558 \mathrm{D}+01 \quad 3.38698 \mathrm{D}-08$ 1.98438D-02 $\begin{array}{lllll}0.9995520 & 6.22030 D+01 & 3.33772 D-08 & 1.85446 D-02\end{array}$

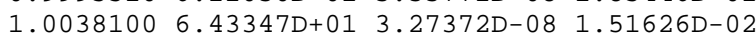
$0.99321206 .59996 \mathrm{D}+01 \quad 3.30013 \mathrm{D}-08$ 1.39555D-02 $\begin{array}{lllll}1.0008800 & 7.39520 D+01 & 3.26008 D-08 & 1.26342 D-02\end{array}$ $0.99934107 .48647 \mathrm{D}+01 \quad 3.27038 \mathrm{D}-08$ 9.81432D-03

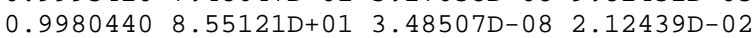
$0.99313608 .54121 \mathrm{D}+01 \quad 3.47320 \mathrm{D}-08$ 2.18092D-02

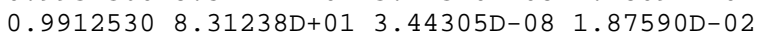
$\begin{array}{llll}1.0019800 & 8.29223 D+01 & 3.43249 D-08 & 1.88151 D-02\end{array}$ $\begin{array}{llll}1.0007900 & 8.22557 D+01 & 3.37026 D-08 & 1.84067 D-02\end{array}$ $\begin{array}{llll}1.0014800 & 8.16694 D+01 & 3.34007 D-08 & 1.71237 D-02\end{array}$ $0.9981630 \quad 8.08293 \mathrm{D}+01 \quad 3.34690 \mathrm{D}-08$ 1.46520D-02 $0.99815707 .33095 D+01 \quad 3.41638 D-08 \quad 2.80263 D-02$ $0.99620607 .22783 D+01 \quad 3.36960 D-08 \quad 2.66013 D-02$ $0.99648006 .61079 \mathrm{D}+01 \quad 3.40354 \mathrm{D}-08$ 2.77298D-02 $0.99328704 .17040 D+01 \quad 3.49662 D-08$ 1.96219D-02 $\begin{array}{llll}1.0001100 & 4.45835 D+01 & 3.46365 D-08 & 2.18025 D-02\end{array}$ $0.99871704 .80663 \mathrm{D}+01 \quad 3.44921 \mathrm{D}-08$ 1.90779D-02 $\begin{array}{llll}1.0010000 & 5.06511 D+01 & 3.43741 D-08 & 1.63281 D-02\end{array}$ $\odot .99493605 .24789 D+01 \quad 3.40498 D-08$ 1.95693D-02 $0.9944640 \quad 5.45572 \mathrm{D}+01 \quad 3.40434 \mathrm{D}-08$ 1.48513D-02 $0.99585606 .17211 D+01 \quad 3.39471 D-08$ 1.46471D-02 $\begin{array}{llll}1.0032800 & 6.97741 D+01 & 3.38265 D-08 & 1.61194 D-02\end{array}$ $\begin{array}{llll}1.0035700 & 8.25839 \mathrm{D}+01 & 3.37188 \mathrm{D}-08 & 1.76494 \mathrm{D}-02\end{array}$ $\begin{array}{llll}1.0005600 & 8.80630 D+01 & 3.41360 D-08 & 1.82805 D-02\end{array}$ $0.99840905 .30919 \mathrm{D}+01 \quad 3.39280 \mathrm{D}-08$ 1.81988D-02 $\begin{array}{llll}1.0005300 & 5.27207 D+01 & 3.43867 D-08 & 1.94161 D-02\end{array}$ $\begin{array}{llll}1.0013900 & 6.57250 \mathrm{D}+01 & 3.40984 \mathrm{D}-08 & 1.52066 \mathrm{D}-02\end{array}$ $\begin{array}{llll}1.0040900 & 7.15408 D+01 & 3.41786 \mathrm{D}-08 & 1.95867 \mathrm{D}-02\end{array}$ $\begin{array}{llll}1.0039200 & 7.54642 \mathrm{D}+01 & 3.39577 \mathrm{D}-08 & 1.75641 \mathrm{D}-02\end{array}$ $\begin{array}{llll}1.00543007 .65689 D+01 & 3.41650 D-08 & 1.83672 D-02\end{array}$ $\begin{array}{lllll}1.0037500 & 6.53705 D+01 & 3.75434 D-08 & 3.07778 D-02\end{array}$ $\begin{array}{llll}1.0040500 & 6.60278 D+01 & 3.67552 D-08 & 2.65350 \mathrm{D}-02\end{array}$ $\begin{array}{llll}1.0068600 & 6.70411 D+01 & 3.59050 D-08 & 2.61849 D-02\end{array}$ $\begin{array}{llll}1.0071300 & 6.76852 D+01 & 3.53190 D-08 & 2.15959 D-02\end{array}$ $\begin{array}{llll}1.0046400 & 6.80222 D+01 & 3.49207 D-08 & 2.13890 D-02\end{array}$ $\begin{array}{llll}1.0063700 & 6.80244 D+01 & 3.47160 D-08 & 2.07644 D-02\end{array}$ $\begin{array}{llll}1.0095400 & 6.84667 D+01 & 3.46611 D-08 & 2.01975 D-02\end{array}$ $\begin{array}{llll}1.0021600 & 3.64905 D+01 & 3.79803 D-08 & 3.13240 D-02\end{array}$ $\begin{array}{llll}1.0035700 & 3.83716 D+01 & 3.64469 D-08 & 2.69020 D-02\end{array}$ $\begin{array}{llll}1.0103400 & 4.38405 D+01 & 3.56999 D-08 & 2.82236 D-02\end{array}$ $\begin{array}{llll}1.0032400 & 4.53286 D+01 & 3.53551 D-08 & 2.24610 D-02\end{array}$ $\begin{array}{llll}1.0029600 & 4.79932 \mathrm{D}+01 & 3.48302 \mathrm{D}-08 & 2.33270 \mathrm{D}-02\end{array}$ $\begin{array}{llll}1.0023500 & 4.88797 D+01 & 3.53220 D-08 & 2.74189 D-02\end{array}$ $\odot .99821505 .32085 D+01 \quad 3.64164 D-08$ 2.07899D-02 $\begin{array}{llll}1.0017600 & 4.28484 \mathrm{D}+01 & 3.70104 \mathrm{D}-08 & 2.28051 \mathrm{D}-02\end{array}$ $\begin{array}{llll}1.0076200 & 4.40713 D+01 & 3.67844 D-08 & 2.20780 D-02\end{array}$ $0.99992004 .80583 D+01 \quad 3.67975 D-08$ 1.85254D-02 $0.99835805 .67225 D+01 \quad 3.69115 D-08 \quad 2.24294 D-02$ $\begin{array}{llll}0.9990730 & 6.05042 D+01 & 3.68317 D-08 & 2.63728 D-02\end{array}$ $\begin{array}{llll}1.0040700 & 4.22059 D+01 & 3.52085 D-08 & 2.14442 D-02\end{array}$ $\odot .99635004 .40111 D+01 \quad 3.56638 D-08$ 2.05270D-02
28.030 25.610 26.390 46.810 117.020 141.840 159.830 193.610 147.140 232.270

84.580

28.420

42.030

30.170

29.970

20.330

30.090

23.830

33.190

26.340

31.500

32.420

27.980

29.060

56.640

51.170

33.690

34.030

36.200

34.480

38.700

29.890

38.530

40.160

24.080

31.310

41.700

28.660

31.280

38.810

34.080

45.750

57.200

45.720

41.590

40.030

32.500

32.590

41.670

39.420

61.380

50.330

58.380

50.640

55.440

45.470

43.060

26.020

32.440

40.470

42.020

34.550

32.450

39.170

38.980

42.000

47.840

41.470

49.890

36.030

27.780 


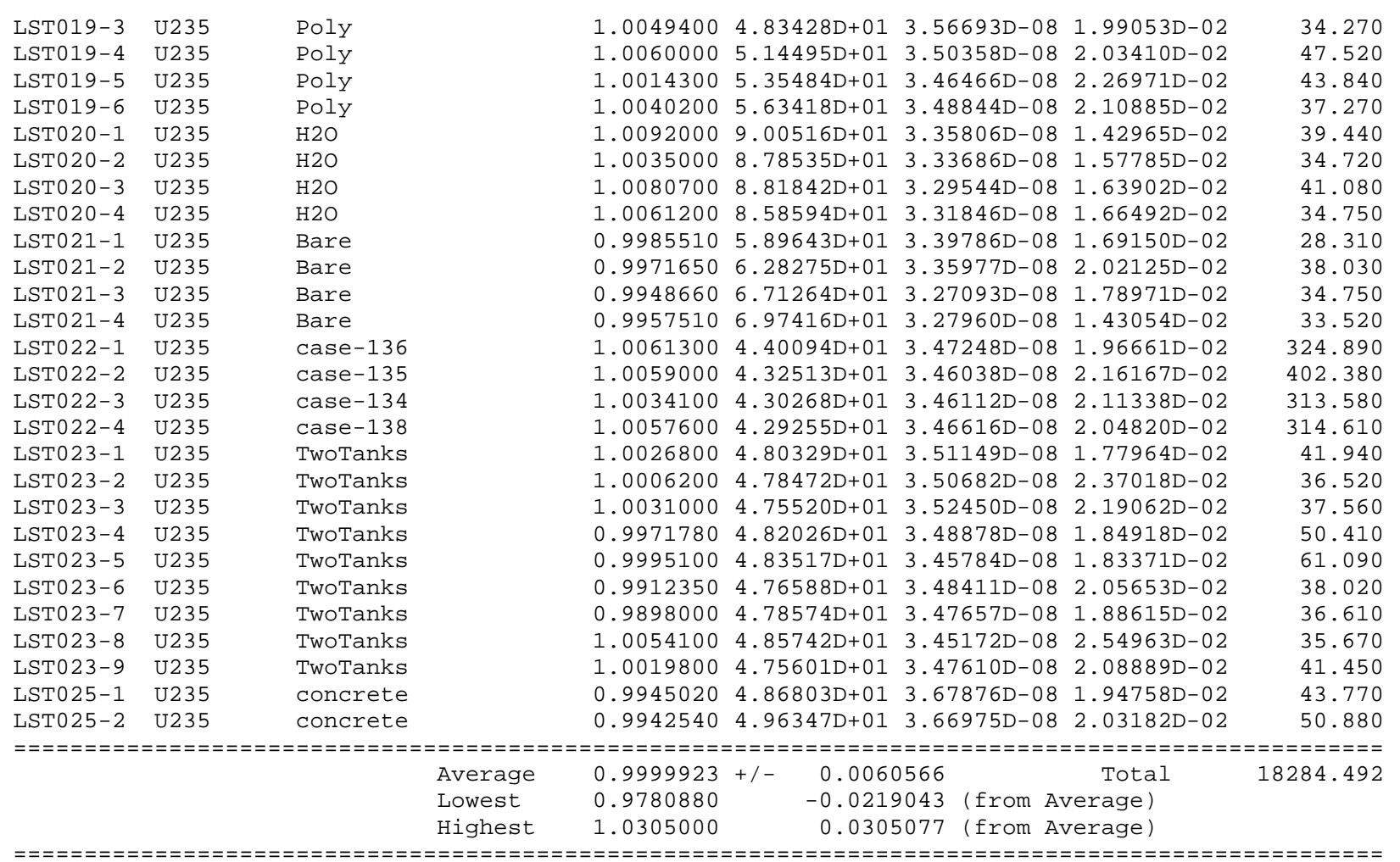

Frequency Distribution for Expected $\mathrm{K}$ Values from $\odot . \odot$ to 2.0

( 0.005 bin width sums)

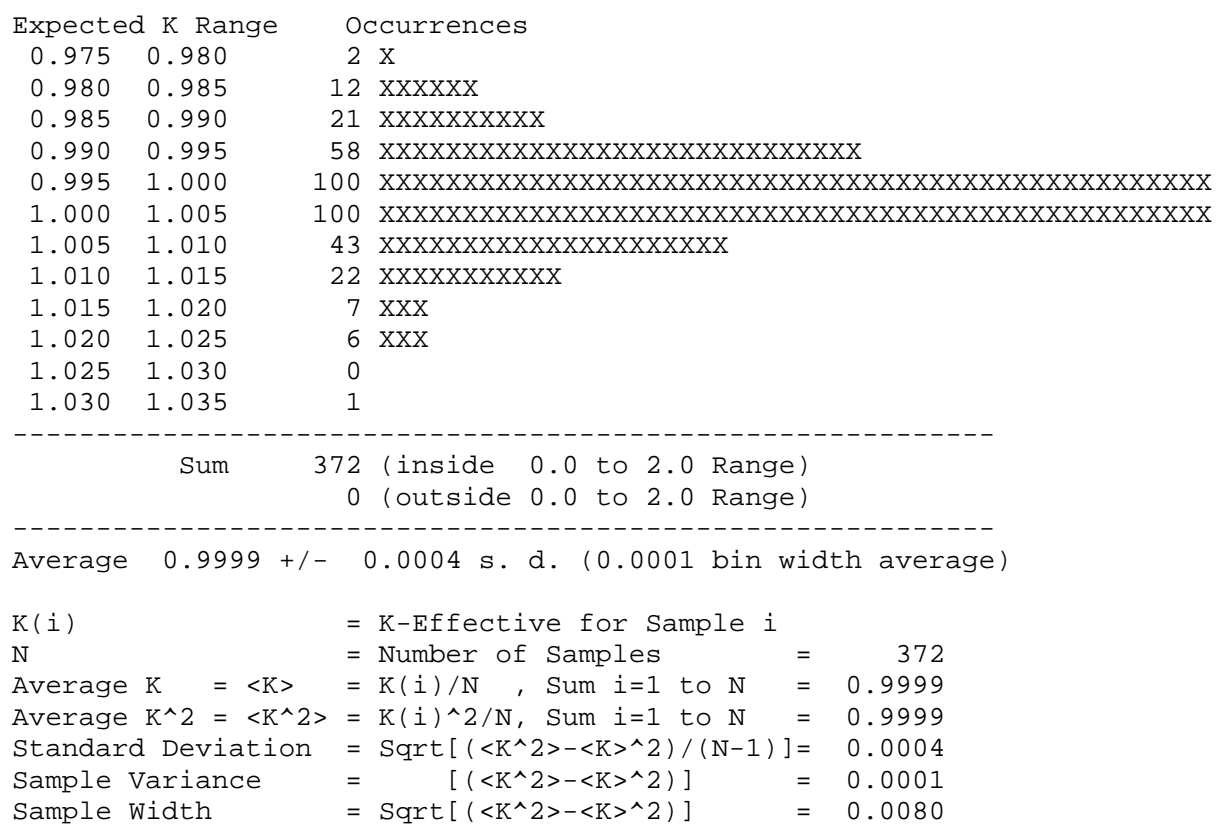

As $\mathrm{N}$ Approaches Infinity Standard Deviation Approaches Zero.

As $N$ Approaches Infinity Sample Width Approaches a Constant.

Confidence Limits

(Occurrences out to +/- 10 times Sample Width)

$\begin{array}{lll}\text { Sample Width Occurrences } & \begin{array}{l}\text { Per-Cent } \\ \text { Occurred }\end{array} & \begin{array}{l}\text { Per-Cent } \\ \text { Nangermal }\end{array}\end{array}$




\begin{tabular}{rrrrr}
-3 & -2 & 13 & 3.495 & 2.140 \\
-2 & -1 & 34 & 9.140 & 13.591 \\
-1 & 0 & 143 & 38.441 & 34.134 \\
0 & 1 & 131 & 35.215 & 34.134 \\
1 & 2 & 40 & 10.753 & 13.591 \\
2 & 3 & 9 & 2.419 & 2.140 \\
3 & 4 & 2 & 0.538 & 0.132 \\
\hline
\end{tabular}

Sum

372 